\title{
DISPERSION ENGINEERING SILICON NITRIDE WAVEGUIDES FOR BROADBAND NONLINEAR FREQUENCY CONVERSION
}

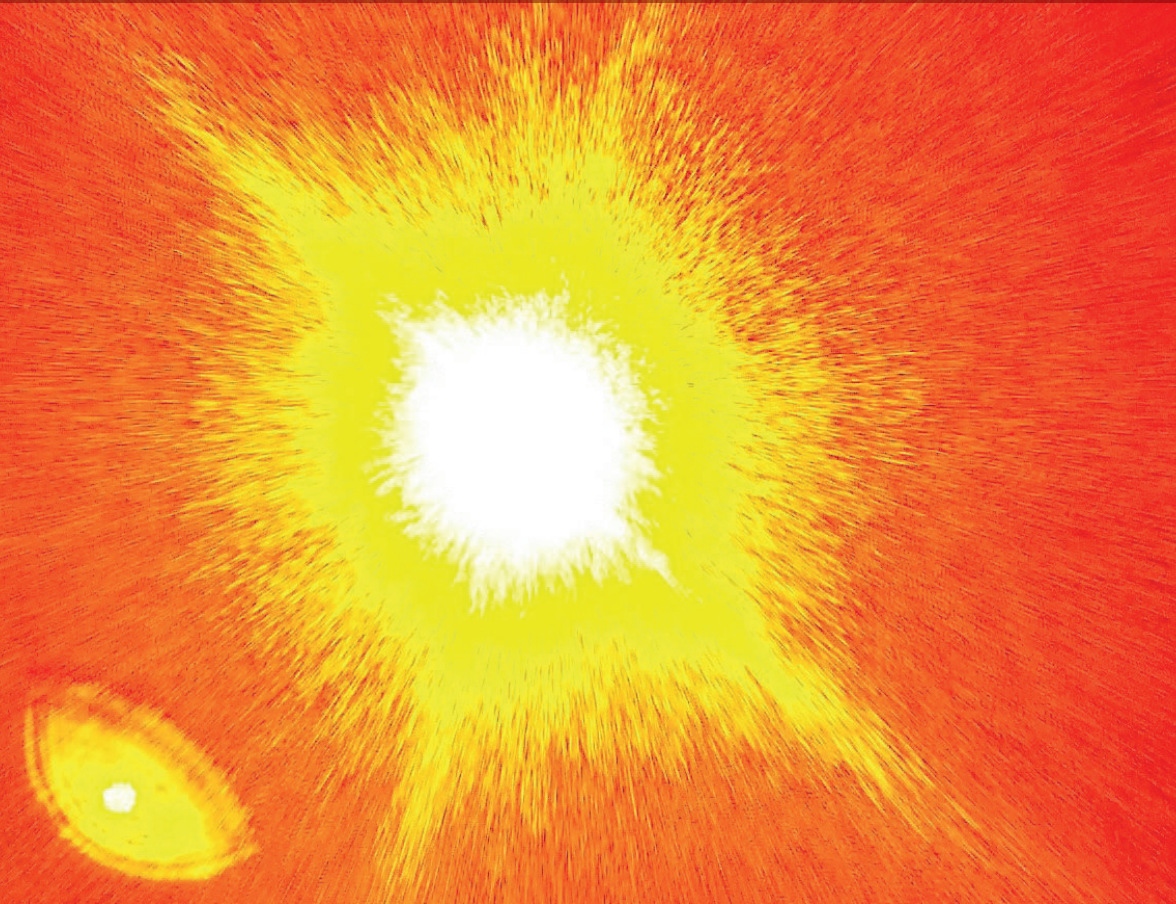

Jörn P. Epping 
DISPERSION ENGINEERING SILICON NITRIDE WAVEGUIDES FOR BROADBAND NONLINEAR FREQUENCY CONVERSION

by

Jörn Philipp Epping 


\section{Ph.D. graduation committee:}

\section{Chairman \& secretary:}

Prof. dr. ir. J.W.M. Hilgenkamp

University of Twente

\section{Promotors:}

Prof. dr. K.-J. Boller

University of Twente

Prof. dr. C. Fallnich

Westfälische Wilhelms-Universität Münster \& University of Twente

\section{Co-promotor:}

Dr. C.J. Lee

University of Twente

\section{Members:}

Prof. dr. A.P. Mosk

University of Twente

Prof. dr. J.L. Herek

University of Twente

Prof. dr. ir. G. Roelkens

Ghent University

Dr. E.A.J.M. Bente

Technische Universiteit Eindhoven

Cover: Camera picture of a supercontinuum beam at the output facet of a $\mathrm{Si}_{3} \mathrm{~N}_{4}$ waveguide (see Chapter 6).

Copyright (C) 2015 J.P. Epping

ISBN: 978-90-365-3939-5

DOI: http://dx.doi.org/10.3990/1.9789036539395

Printed by Gildeprint, Enschede, The Netherlands 


\title{
DISPERSION ENGINEERING SILICON NITRIDE WAVEGUIDES FOR BROADBAND NONLINEAR FREQUENCY CONVERSION
}

\author{
DiSSERTATION
}

to obtain

the degree of doctor at the University of Twente, on the authority of the rector magnificus, prof. dr. H. Brinksma, on account of the decision of the graduation committee, to be publicly defended on Thursday the $3^{\text {rd }}$ September 2015 at 12.45 .

by

\section{Jörn Philipp Epping}

born on 24 October 1984

in Greven, Germany 
This dissertation has been approved by:

Promotors: Prof. dr. K.-J. Boller

Prof. dr. C. Fallnich

The research presented in this thesis was carried out at the Laser Physics and Nonlinear Optics group, Department of Science and Technology, MESA+ Institute for Nanotechnology, University of Twente, P.O. Box 217, 7500 AE Enschede, The Netherlands. This research is supported by the Dutch Technology Foundation STW, which is part of the Netherlands Organization for Scientific Research (NWO) and partly funded by the Ministry of Economic Affairs (project number 11358). 
To Nina. 



\section{Contents}

1 Introduction $\quad \mathbf{1}$

1.1 Thesis outline ..................... 5

2 Theoretical background 11

2.1 Optical waveguides . . . . . . . . . . . . . . . 12

2.1.1 Calculation of waveguide modes . . . . . . . . 12

2.1.2 Optical modes in silicon nitride waveguides . . . . . . 13

2.1.3 Waveguide dispersion ... . . . . . . . . 16

2.2 Nonlinear frequency conversion . . . . . . . . . . . . . . . 19

2.2.1 Four-wave mixing . . . . . . . . . . . . . 21

2.2.2 Self-phase modulation . . . . . . . . . . . . 23

2.2.3 Nonlinear Schrödinger equation . . . . . . . . . . . 25

2.2.4 Supercontinuum generation ........... 26

3 Integrated CARS source based on seeded four-wave mixing in silicon nitride $\quad 31$

3.1 Introduction . . . . . . . . . . . . . . . . . 32

3.2 Integrated CARS source . . . . . . . . . . . . . 33

3.3 Conclusion ........................... 38

4 High confinement, high yield $\mathrm{Si}_{3} \mathrm{~N}_{4}$ waveguides for nonlinear optical applications $\quad 43$

4.1 Introduction . . . . . . . . . . . . . . . . . 44

4.2 Fabrication ..................... 46

4.3 Characterization . . . . . . . . . . . . . . . . . 48

4.4 Conclusion ........................ 50

5 Broadband supercontinuum generation in dispersion engineered stoichiometric silicon nitride waveguides pumped at telecommunication wavelengths 53

5.1 Introduction . . . . . . . . . . . . . . . 54

5.2 Dispersion calculation ................. 55 
5.3 Experimental results . . . . . . . . . . . . . . . . 56

5.4 Theoretical modeling . . . . . . . . . . . . . . . 58

5.5 Conclusion . . . . . . . . . . . . . . . . 61

6 On-chip visible-to-infrared supercontinuum generation with more than $495 \mathrm{THz}$ spectral bandwidth $\quad 67$

6.1 Introduction . . . . . . . . . . . . . . . . . . 68

6.2 Dispersion calculation . . . . . . . . . . . . . . . . . 69

6.3 Experimental results . . . . . . . . . . . . . . . . . . . . 69

6.4 Numerical calculations . . . . . . . . . . . . . . . . . . 72

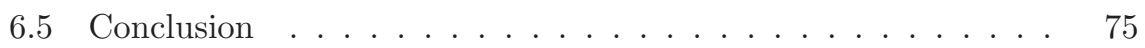

7 Conclusion and outlook $\quad 81$ 


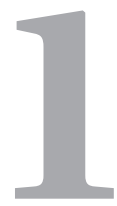

Introduction 
The General Assembly of the United Nations proclaimed 2015 as the International Year of Light and Light-based Technologies [1]. One of the reasons was that light is considered to be of vital importance for existing and future advances in numerous fields in science, industry, and society. Light obtained this position because many, if not all, of its properties can be controlled. For example, polarization control enabled technologies such as LCDs (liquid crystal displays), while the specific properties of light produced by lasers (e.g. coherence, monochromaticity, and extremely high intensities) enabled numerous applications such as long-distance and high-speed communications [2], micro material processing [3], or nonlinear optical applications [4].

During the last fifteen years new light sources based on supercontinuum generation (SCG) were developed. By using SCG the coherence properties of a laser can be maintained and broadband spectra with a deterministic relation of the phases can be obtained by nonlinear frequency conversion. SCG can occur when an ultrashort pulse of laser light propagates through a material. The unique coherence properties of these broadband spectra allow the generation of frequency combs, which are sometimes also referred to as optical rulers, which enabled ultra-precise optical frequency metrology [5]. Other applications of these frequency combs include newly developed spectroscopic methods such as dual-comb spectroscopy [6] and optical coherence tomography [7]. Especially, for particular spectroscopic applications in the life sciences it is desirable to generate such spectra in the visible wavelength range [8]. To date, most of these light sources are based on optical silica fibers. In order to be more energy efficient as well as to achieve a smaller device footprint, it is desirable to establish such light sources using integrated waveguide platforms.

Furthermore, waveguide platforms that are compatible with microfluidic applications, for so-called lab-on-a-chip setups, would greatly benefit from an on-chip broad bandwidth light source. Such a waveguide platform is preferably based on a reliable fabrication to produce highly-integrated, highly-functional, economical sensors. On the other hand, lab-on-a-chip setups would also require a waveguide platform with low losses because integrated optical functions such as light generation, filtering, interaction, and detection require relative long on-chip propagation lengths. Of the main integrated waveguide platforms, stoichiometric silicon nitride $\left(\mathrm{Si}_{3} \mathrm{~N}_{4}\right)$ based waveguides have a transparency range from the visible to well into the infrared [9], demonstrated ultra-low propagation losses [10], and are compatible to microfluidic applications [11]. Hence, $\mathrm{Si}_{3} \mathrm{~N}_{4}$-based waveguides are a promising and versatile platform to use to study nonlinear frequency conversion in integrated waveguides.

In general, nonlinear frequency conversion in dielectric materials can occur when light is so intense that the response of the material to the electric field of the light is not linear anymore. At high intensities, higher orders of the dielectric material response have to be taken into account, which results in the generation of new frequencies. The magnitude of the conversion depends on the strength and order of nonlinearity of the material (see Chapter 2.2) and the light intensity that induces the nonlinear response. However, in general the 
nonlinear conversion is a weak effect. To increase the efficiency of the conversion process a material with a high nonlinearity is selected and typically lasers are used to reach intensities high enough to efficiently generate new frequencies. A further requirement for efficient nonlinear conversion is that, for each of the frequencies, the electric field of the newly generated light has to interfere constructively with the already propagating field. Constructive interference or so-called phase matching can be achieved, e.g., via providing suitable dispersion or birefringence. Therefore, the wavelength of the generated light in a nonlinear process can be controlled, e.g., by designing the dispersive properties of the medium.

The efficiency of nonlinear optical effects can be further increased by using optical waveguides, as a high light intensity can be maintained over propagation distances that are hard to realize using bulk optics. A well-known optical waveguide is the optical glass fiber, which is, e.g., used in optical telecommunications [2]. In a simplified ray-optic picture the light is guided in a waveguide by total internal reflection within a region with a high refractive index, called the waveguide core, that is surrounded by a region with a lower refractive index, called the cladding. To control the dispersion in a waveguide the geometry of the waveguide core can be changed to obtain phase matching for particular frequencies. Especially, integrated waveguides can enable a high intensity in the core at relatively low pump powers, when compared to an optical fiber, due to tight confinement of the light to the core that has a high refractive index compared to the cladding. The potential for nonlinear optical applications was already investigated at the beginning of research on integrated photonic circuits [12]. However, only recent advances in fabrication technology allowed for high-quality integrated waveguides. A further way to increase the efficiency of nonlinear processes is to select an integrated platform consisting of materials that have a large nonlinearity. For example, nonlinear integrated waveguides based on chalcogenides have a high nonlinearity [13] and were used to demonstrate broadband nonlinear frequency conversion [14]. But also new material platforms are being investigated and have a huge potential, e.g., diamond-based waveguides [15] have exceptional properties such as a high refractive index and broad transparency window or InGaP (indium gallium phosphide) membrane waveguides that offer an ultra high nonlinearity [16]. By selecting integrated photonic platforms that offer compatibility with CMOS (complementary metaloxide-semiconductor) fabrication, devices can be processed by well-established and highly reliable fabrication methods and can, therefore, be brought into mass production easier. The most prominent of the CMOS-compatible platforms is silicon-on-insulator and due to the high nonlinearity of silicon, it is predominantly used in nonlinear integrated photonics [17]. The drawback of silicon is its low band gap (corresponding to a wavelength of $\approx 1 \mu \mathrm{m}$ ), which ultimately limits the generated wavelengths to the infrared. Furthermore, due to the high intensities required for nonlinear frequency conversion, nonlinear losses can arise within the material, e.g., two-photon absorption, when pumped in the near-infrared (below a wavelength of $2.2 \mu \mathrm{m}$ ). In order to use lower pump 
wavelengths such as the telecommunication C-band also waveguides based on hydrogenated amorphous silicon $(\mathrm{a}-\mathrm{Si}: \mathrm{H})$ can be used as it exhibits a lower two-photon absorption than crystalline silicon [18]. However, to extend the spectral range to lower wavelengths, materials with a higher band gap energy are required.

Integrated platforms that offer CMOS-compatibility as well as a high band gap are silicon nitride and doped silica [19] and both have been shown to be promising for nonlinear integrated optics. Especially, stoichiometric silicon nitride $\left(\mathrm{Si}_{3} \mathrm{~N}_{4}\right)$ waveguides, such as used in this thesis, have been reported to have a low waveguide loss [10] allowing for long interaction lengths and complex integrated photonic circuits [20]. Its wide transparency range from the visible blue at $400 \mathrm{~nm}$ into the infrared until $2400 \mathrm{~nm}$ [9], together with its compatibility with microfluidic channels [11] and, most important, its relatively high nonlinearity [21] makes $\mathrm{Si}_{3} \mathrm{~N}_{4}$-based waveguides an excellent platform for integrated nonlinear optics, in particular nonlinear frequency conversion.

To date, nonlinear frequency conversion with a broad bandwidth focuses on supercontinuum generation and the closely related generation of frequency combs through cascaded four-wave mixing (FWM) in microresonators. The latter are also called hyper optical parametric oscillators (OPOs) and have been realized in a number of platforms [22], including $\mathrm{Si}_{3} \mathrm{~N}_{4}$ [23]. Hyper OPOs are relying to a large extend on a low linear propagation loss (i.e., low absorption and scattering losses). This enables a high power build-up inside a high-Q resonator when using a continuous-wave pump laser with an ultra-narrow spectral line width. Due to the small size of the microresonators [22], the mode spacing in such spectra is typically in the order of 10 to $1000 \mathrm{GHz}$. The cascaded FWM of individual resonator modes of the microresonator results in comb-like spectra, which are sometimes referred to as Kerr combs, and the overall spectral bandwidth can become rather large, spanning more than an octave [28]. In CMOS-compatible waveguides, Kerr combs have been reported in the nearinfrared in silicon nitride [23] and doped silica [24] when pumped at $1.5 \mu \mathrm{m}$ and in silicon when pumped at $2.6 \mu \mathrm{m}$ [25]. A priori, the individual comb lines may have a random phase relation if the cascaded FWM starts from vacuum fluctuations. On the other hand, if a temporally stable mutual phase relation exists between the comb modes, then in the time domain the output is in the form of a train of pulses with ultrashort duration. This type of phasing was observed, e.g., in $\mathrm{Si}_{3} \mathrm{~N}_{4}$ microresonators [26, 27] and was interpreted as a formation of cavity solitons. Despite the successful demonstration, cascaded FWM in microresonators still faces some challenges. The frequencies at the edge of the Kerr comb lack sufficient power for straight forward self-referencing. An additional limitation is that the obtained cavity enhancement and, hence, the overall spectral bandwidth of generated Kerr combs strongly depends on the waveguide loss. Therefore, it is technologically extremely challenging to directly generate wavelengths in the visible due to the drastic increase of loss for shorter wavelengths.

A more efficient way to generate broad bandwidths with a determined phase 
relation in integrated waveguides is SCG using ultrashort pulses in the femtosecond range $(\approx 100 \mathrm{fs})$. One reason is that by injecting an ultrashort pulse into an integrated waveguide the required peak intensity for highly efficient nonlinear conversion within a short propagation length is easily provided without the need of a resonator, while the average laser power is kept low. The latter avoids thermal effects to a large extent. Another reason is that the bandwidth of SCG is less prone to narrowing through the presence of waveguide loss since, at high intensities, the required interaction length can be reduced to a few millimeters. Hence, bandwidths of multiple octaves were achieved in integrated waveguide structures such as in highly nonlinear soft glass [14] and lithium niobate [29]. In CMOS-compatible silicon waveguides octave-spanning spectra in the mid-infrared [30, 31] were reported and determined to be highly coherent [31]. However, due to the small band gap of silicon, these bandwidths cannot be obtained at pump wavelengths below $2 \mu \mathrm{m}$. To shift the edge of the generated light to shorter wavelengths, laser sources with a shorter pump wavelength have to be employed. This is accomplished in this thesis and in other works [32-34] by using $\mathrm{Si}_{3} \mathrm{~N}_{4}$ waveguides. Using various pump wavelengths, octave-spanning supercontinuum spectra were obtained, and the supercontinuum was shown to be highly coherent [34]. In this thesis, an ytterbium-doped fiber laser is used to generate the widest supercontinuum to date in dispersion engineered $\mathrm{Si}_{3} \mathrm{~N}_{4}$ waveguides.

\subsection{Thesis outline}

In this thesis, we describe the nonlinear optical frequency conversion in integrated silicon nitride waveguides. For nonlinear conversion, we use the third order nonlinearity, which is the dominant nonlinearity present in silicon nitride. Here, we use four-wave mixing (FWM) and supercontinuum generation (SCG) to achieve a broadband conversion of optical spectra.

To assess the potential of $\mathrm{Si}_{3} \mathrm{~N}_{4}$ waveguides for nonlinear frequency conversion and to compare experimental results with theory, we introduce the generalized nonlinear Schrödinger equation as a numerical model in Chapter 2, which is further extended in Chapter 6 to include multiple transverse modes to describe the nonlinear interaction of the pump light.

Driven by applications for nonlinear coherent anti-Stokes Raman scattering (CARS), we propose a conversion scheme using four-wave mixing in Chapter 3. Based on a numerical model, we show that an integrated light source with broadband spectral control is feasible using silicon nitride waveguides. The generated spectrum is controlled by using FWM of a high power pump laser with a tunable continuous-wave seed laser.

To provide phase matching for both FWM and SCG, it is required that the silicon nitride waveguides exhibit anomalous dispersion for the pump wave. However, the fabrication of waveguides with anomalous dispersion from a single $\mathrm{Si}_{3} \mathrm{~N}_{4}$ layer is challenging due to material stress, which leads to the formation 
of cracks in the $\mathrm{Si}_{3} \mathrm{~N}_{4}$ layer. To avoid the formation of cracks, which results in a low device yield, we employed a novel high-yield fabrication method that provides the large core waveguides required for nonlinear frequency conversion. The fabrication and characterization of these crack-free $\mathrm{Si}_{3} \mathrm{~N}_{4}$ waveguides are presented in Chapter 4.

Finally, we use supercontinuum generation to demonstrate the broad bandwidth that can be achieved by nonlinear optical processes in our novel silicon nitride waveguides. In Chapter 5 , we use an erbium-doped fiber laser and generate a supercontinuum of more that $700 \mathrm{~nm}$ (at a $-30 \mathrm{~dB}$ level) and extrapolate that a bandwidth of more than $1200 \mathrm{~nm}$ can be generated at higher pump pulse energies. Furthermore, we show via calculations that the spectra are fully coherent over their entire bandwidth. In Chapter 6 this bandwidth is surpassed by a supercontinuum spanning over more than $1600 \mathrm{~nm}$ originating from an ytterbium-doped fiber laser at $1064 \mathrm{~nm}$. The generated spectrum fills almost the whole transmission window of silicon nitride waveguides by spanning from the visible blue $(470 \mathrm{~nm})$ to the infrared $(2100 \mathrm{~nm})$ with a frequency range of $495 \mathrm{THz}$. To date, this is the broadest supercontinuum that has ever been generated in any integrated waveguide. 


\section{Bibliography}

[1] General Assembly of the United Nations, "International Year of Light and Light-based Technologies, 2015," resolution A/RES/68/221 (2013).

[2] G. P. Agrawal, Fiber-Optic Communication Systems, 4th ed., (Wiley$\mathrm{VCH}, 2010)$.

[3] W. Steen, K. G. Watkins, and J. Mazumder, Laser Material Processing, (Springer, 2010).

[4] G. P. Agrawal, Applications of Nonlinear Fiber Optics, (Academic, 2010).

[5] T. Udem, R. Holzwarth, and T. W. Hänsch, "Optical frequency metrology," Nature 416, 233-237 (2002).

[6] B. Bernhardt, A. Ozawa, P. Jacquet, M. Jacquey, Y. Kobayashi, T. Udem, R. Holzwarth, G. Guelachvili, T. W. Hänsch, and N. Picqué, "Cavityenhanced dual-comb spectroscopy," Nature Photon. 4, 55-57 (2010).

[7] A. F. Fercher, W. Drexler, C. K. Hitzenberger, and T. Lasser, "Optical coherence tomographyprinciples and applications," Rep. Prog. Phys. 66, 239 (2003).

[8] J. Yi, Q. Wei, W. Liu, V. Backman, and H. F. Zhang, "Visible-light optical coherence tomography for retinal oximetry," Optics Lett. 38(11), 17961798 (2013).

[9] K. Wörhoff, R. G. Heideman, A. Leinse, and M. Hoekman, "TriPleX: a versatile dielectric photonic platform," Adv. Opt. Mater. 4(2), 189-207 (2015).

[10] J. F. Bauters, M. J. R. Heck, D. John, D. Dai, M.-C. Tien, J. S. Barton, A. Leinse, R. G. Heideman, D. J. Blumenthal, and J. E. Bowers, "Ultralow-loss high-aspect-ratio $\mathrm{Si}_{3} \mathrm{~N}_{4}$ waveguides," Optics Express 19(4), 316374 (2011).

[11] A. Ymeti, J. S. Kanger, J. Greve, G. A. J. Besselink, P. V. Lambeck, R. Wijn, and R. G. Heideman, "Integration of microfluidics with a fourchannel integrated optical Young interferometer immunosensor," Biosens. Bioelectron. 20(7), 1417-1421 (2005). 
[12] G. I. Stegeman and R. H. Stolen, "Waveguides and fibers for nonlinear optics," J. Opt. Soc. Am. B 6(4), 652 (1989).

[13] B. J. Eggleton, B. Luther-Davies, and K. Richardson, "Chalcogenide photonics," Nature Photon. 5(12), 141-148 (2011).

[14] Y. Yu, X. Gai, P. Ma, D. Y. Choi, Z. Yang, R. Wang, S. Debbarma, S. J. Madden, and B. Luther-Davies, "A broadband, quasi-continuous, midinfrared supercontinuum generated in a chalcogenide glass waveguide," Laser Photon. Rev. 798(5), 792-798 (2014).

[15] B. J. M. Hausmann, I. Bulu, V. Venkataraman, P. Deotare, and M. Lončar, "Diamond nonlinear photonics," Nature Photon. 8(4), 1-6 (2014).

[16] U. Dave, C. Ciret, S.-P. Gorza, S. Combrié, A. De Rossi, F. Raineri, G. Roelkens, and B. Kuyken "Dispersive-wave-based octave-spanning supercontinuum generation in InGaP membrane waveguides on a silicon substrate," Optics Lett. 40(15), 3584 (2015).

[17] J. Leuthold, C. Koos, and W. Freude, "Nonlinear silicon photonics," Nature Photon. 4(8), 535-544 (2010).

[18] F. Leo, J. Safioui, B. Kuyken, G. Roelkens, and S.-P. Gorza, "Generation of coherent supercontinuum in a-Si:H waveguides: experiment and modeling based on measured dispersion profile," Optics Express 22(23), 28997 (2014).

[19] D. J. Moss, R. Morandotti, A. L. Gaeta, and M. Lipson, "New CMOScompatible platforms based on silicon nitride and Hydex for nonlinear optics," Nature Photon. 7(8), 597-607 (2013).

[20] C. G. H. Roeloffzen, L. Zhuang, C. Taddei, A. Leinse, R. G. Heideman, P. W. L. van Dijk, R. M. Oldenbeuving, D. A. I. Marpaung, M. Burla, and K.-J. Boller, "Silicon nitride microwave photonic circuits," Optics Express 21(19), 22937-22961 (2013).

[21] K. Ikeda, R. E. Saperstein, N. Alic, and Y. Fainman, "Thermal and Kerr nonlinear properties of plasma-deposited silicon nitride/silicon dioxide waveguides," Optics Express 16(17), 12987-94 (2008).

[22] T. J. Kippenberg, R. Holzwarth, and S. A. Diddams, "Microresonatorbased optical frequency combs," Science 332(6029), 555-559 (2011).

[23] J. S. Levy, A. Gondarenko, M. A. Foster, A. C. Turner-Foster, A. L. Gaeta, and M. Lipson, "CMOS-compatible multiple-wavelength oscillator for on-chip optical interconnects," Nature Photon. 4(1), 37-40 (2010).

[24] L. Razzari, D. Duchesne, M. Ferrera, R. Morandotti, S. Chu, B. E. Little, and D. J. Moss, "CMOS-compatible integrated optical hyper-parametric oscillator," Nature Photon. 4, 41-45 (2009). 
[25] A. G. Griffith, R. K. Lau, J. Cardenas, Y. Okawachi, A. Mohanty, R. Fain, Y. H. D. Lee, M. Yu, C. T. Phare, C. B. Poitras, A. L. Gaeta, and M. Lipson, "Silicon-chip mid-infrared frequency comb generation," Nature Commun. 6, 6299 (2015).

[26] K. Saha, Y. Okawachi, B. Shim, J. S. Levy, R. Salem, A. R. Johnson, M. A. Foster, M. R. E. Lamont, M. Lipson, and A. L. Gaeta, "Modelocking and femtosecond pulse generation in chip-based frequency combs," Optics Express 21(1), 1335-43 (2013).

[27] V. Brasch, T. Herr, M. Geiselmann, G. Lihachev, M. H. P. Pfeiffer, M. L. Gorodetsky, and T. J. Kippenberg, "Photonic chip based optical frequency comb using soliton induced Cherenkov radiation," arXiv:1410.8598, (2014).

[28] Y. Okawachi, K. Saha, J. S. Levy, Y. H. Wen, M. Lipson, and A. L. Gaeta, "Octave-spanning frequency comb generation in a silicon nitride chip," Optics Lett. 36(17), 3398-3400 (2011).

[29] C. R. Phillips, C. Langrock, J. S. Pelc, M. M. Fejer, J. Jiang, M. E. Fermann, and I. Hartl, "Supercontinuum generation in quasi-phase-matched $\mathrm{LiNbO}_{3}$ waveguide pumped by a Tm-doped fiber laser system," Optics Lett. 36(19), 3912-4 (2011).

[30] R. K. W. Lau, M. R. E. Lamont, A. G. Griffith, Y. Okawachi, M. Lipson, and A. L. Gaeta, "Octave-spanning mid-infrared supercontinuum generation in silicon nanowaveguides," Optics Lett. 39(15), 4518-21 (2014).

[31] B. Kuyken, T. Ideguchi, S. Holzner, M. Yan, T. W. Hänsch, J. Van Campenhout, P. Verheyen, S. Coen, F. Leo, R. Baets, G. Roelkens, and N. Picqué, "An octave-spanning mid-infrared frequency comb generated in a silicon nanophotonic wire waveguide," Nature Commun. 6, 6310 (2015).

[32] R. Halir, Y. Okawachi, J. S. Levy, M. A. Foster, M. Lipson, and A. L. Gaeta, "Ultrabroadband supercontinuum generation in a CMOScompatible platform," Optics Lett. 37(10), 1685-7 (2012).

[33] H. Zhao, B. Kuyken, S. Clemmen, F. Leo, A. Subramanian, A. Dhakal, P. Helin, S. Severi, E. Brainis, G. Roelkens, and R. Baets, "Visible-to-nearinfrared octave spanning supercontinuum generation in a silicon nitride waveguide," Optics Lett. 40(10), 2177 (2015).

[34] A. S. Mayer, A. Klenner, A. R. Johnson, K. Luke, M. R. E. Lamont, Y. Okawachi, M. Lipson, A. L. Gaeta, and U. Keller, "Frequency comb offset detection using supercontinuum generation in silicon nitride waveguides," Optics Express 23(12), 15440 (2015). 



\section{2 \\ Theoretical background}

In this chapter the theory of linear and nonlinear optics of guided waves is discussed and applied to silicon nitride-based waveguides. In the first section we recall the linear properties of optical waveguides and, specifically, the characteristics of silicon nitride waveguides. In the second section nonlinear optics is introduced with an emphasis on the third-order nonlinearity, which is the predominant nonlinearity in silicon nitride. Furthermore, we discuss two nonlinear frequency conversion schemes for silicon nitride waveguides. The first scheme is seeded four-wave mixing and is used in Chapter 3 to propose a tunable integrated light source for nonlinear spectroscopy and microscopy. The second scheme is supercontinuum generation by ultrashort pulses, which is mostly due to spectral broadening from self and cross-phase modulation of the injected pump pulse. This second scheme is used in Chapter 5 and 6 to create broadband spectra. At the end of this section, the nonlinear Schrödinger equation is introduced, which forms the basis of the numerical model used in Chapter 3, 5, and 6 to numerically model the nonlinear pulse propagation in silicon nitride waveguides. 


\subsection{Optical waveguides}

In its simplest form, an optical waveguide consists of two dielectric materials with different refractive indices, $n_{a}$ and $n_{b}\left(n_{a}>n_{b}\right)$. Using a ray-optics picture, light is guided by total internal reflection at the core-cladding interface, where the core is made out of the higher index material $\left(n_{a}\right)$ and is surrounded by the lower index material $\left(n_{b}\right)$, the so-called cladding. When the core is surrounded in two dimensions ( $x$ and $y$ ), both typically with a length scale of the order of the wavelength of the light, light becomes confined in these two dimensions whereas propagation occurs in the $z$-direction over long distances. Furthermore, the confinement of light is conserved by the waveguide during propagation. This is an advantage over free-space optics, where light confinement, such as in a focus is followed by beam expansion via diffraction. The most common optical waveguide is the optical fiber, which has revolutionized telecommunication and together with the invention of the CCD sensor was awarded the Nobel prize in physics in 2009. In this chapter, we consider an integrated optical waveguide with a rectangular silicon nitride core with $n_{a} \approx 2.0$ and $\mathrm{a} \mathrm{SiO}_{2}$ cladding with $n_{b} \approx 1.45$, and (mostly) a wavelength, $\lambda$, of $1064 \mathrm{~nm}$, which is, e.g., readily available from Ytterbium or Neodymiumdoped laser media. The dimensions of the waveguide core in this thesis are in the same order of magnitude in size, about $1 \mu \mathrm{m}$ by $1 \mu \mathrm{m}$. At such small dimensions comparable to the wavelength, the ray-optics picture is no longer valid and light has to be considered as an electromagnetic wave.

\subsubsection{Calculation of waveguide modes}

Waveguides support a finite number of modes, which are certain spatial eigensolutions of the Maxwell's equations for the boundary conditions given via the waveguide properties. These eigensolutions can be represented via the spatial distributions of the electromagnetic field, $\boldsymbol{E}(x, y)$. Here, we consider waveguides made of two dielectric, non-magnetic materials in the absence of free charge and current, which is valid for silicon nitride and silica. To find an optical mode, it is sufficient to find either the electric field or magnetic field as one can be derived from the other using Maxwell's equations. Typically, one sets up the problem to solve for the electric field, $\boldsymbol{E}$. Under continuity conditions at the boundaries of the waveguide core and the condition of a translation invariant amplitude in $z$-direction of the electric field, $\boldsymbol{E}$, the optical modes are eigenstates of the modal vector wave equation. The equation can be written in the following form [1]:

$$
\left(\nabla_{x, y}^{2}+k^{2} n^{2}-\beta^{2}\right) \boldsymbol{E}=-\left(\nabla_{x, y}+i \beta \hat{\boldsymbol{z}}\right)\left(\boldsymbol{E}_{x, y} \cdot \nabla_{x, y} \ln n^{2}\right),
$$

where $k$ is the wavenumber of the light field in vacuum, $n(x, y)$ the transverse distribution of the refractive index for the waveguide, $\beta$ the propagation constant of the mode and $\hat{z}$ is the unit vector in the propagation direction $z$. For a 
detailed derivation from Maxwell's equations, the reader is referred to standard textbooks [1].

From Eq. 2.1, the electric field distribution and the propagation constant of the individual modes can be obtained for a specific vacuum wavelength, $\lambda$, and a given refractive index distribution, $n(x, y)$. The effective refractive index of each mode is defined as $n_{\mathrm{eff}}=\beta \lambda / 2 \pi$. Solving Eq. 2.1 reveals that the value for $n_{\text {eff }}$ is a weighted average of core and cladding indices with the mode field distribution as weight factor. This results in a higher $n_{\text {eff }}$ for modes that are more confined to the waveguide than for modes where a larger fraction of the electric field is located in the cladding.

While there are analytical methods to approximate the optical mode, e.g., the Marcatili method [2], we use a finite element (FEM) solver to calculate the optical modes in our waveguides. By using FEM solvers realistic waveguide shapes with a more complex refractive index distribution can be calculated.

In an FEM solver, Eq. 2.1 is solved by approximating the two dimensional surface of the waveguide cross section, consisting of core and cladding, into a set of elements. The calculation accuracy increases with the number of elements, albeit at the expense of computational speed. In our case, the density of elements was chosen to be higher in the waveguide core than in the cladding, since most of the light is propagating in the waveguide core. An appropriate distribution of mesh elements is found by applying convergence tests. In this thesis, we used two different FEM solvers, namely COMSOL Multiphysics ${ }^{1}$ and an FEM solver based on Matlab published by Fallahkhair et al. [3].

\subsubsection{Optical modes in silicon nitride waveguides}

In this section the basic properties of the optical modes for a typical silicon nitride waveguide are discussed. For definiteness and to provide typical values, we choose a waveguide core with $w=1.0 \mu \mathrm{m}$ and $h=0.25 \mu \mathrm{m}$, such as available using standard fabrication methods. Here, we consider a wavelength of $1064 \mathrm{~nm}$ for which this waveguide is multi-mode. Figure 2.1 shows the absolute value of the calculated electric field distribution of all the modes that are supported by this waveguide. These waveguides support modes that are polarized in both the $x$ and $y$ directions. By convention, the modes mainly polarized in the $\mathrm{x}$-direction are called TE modes and the modes mainly polarized in the y-direction are called TM modes. The naming of the modes is as follows: the subscripts correspond to the number of electric field nodes, where the first index corresponds to the $\mathrm{x}$-axis and the second to the $\mathrm{y}$-axis.

It can be seen that the distribution of the electric field is clearly different for the different modes. Apart from the features of the distribution, the electric field is more or less strongly confined by the waveguide. The degree of confinement can be expressed as an effective mode area, $A_{\text {eff, }}$ which is defined as [4]:

\footnotetext{
${ }^{1}$ https://www.comsol.com/
} 

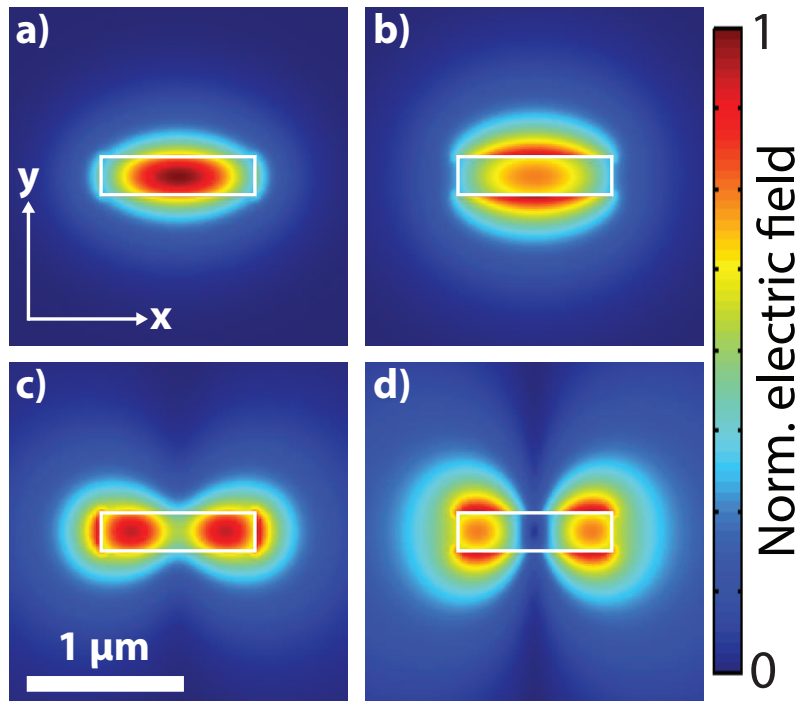

Figure 2.1: Electric field distribution in absolute values of optical modes calculated for a silicon nitride waveguide with a height, $h$, of $0.25 \mu \mathrm{m}$ and width, $w$, of $1.0 \mu \mathrm{m}$ for a wavelength of $1064 \mathrm{~nm}$. The fundamental modes (a) $T E_{00}$ mode $\left(n_{\text {eff }}=1.66\right)$ and (b) $T M_{00}\left(n_{\text {eff }}=\right.$ $1.57)$ and the higher-order modes (c) $T E_{10}\left(n_{\text {eff }}=1.51\right)$ and $(d) T M_{10}$ $\left(n_{\text {eff }}=1.46\right)$. 


$$
A_{\mathrm{eff}}=\frac{\left(\iint_{-\infty}^{+\infty}|E(x, y)|^{2} d x d y\right)^{2}}{\iint_{-\infty}^{+\infty}|E(x, y)|^{4} d x d y}
$$

For the modes shown in Fig. $2.1 A_{\text {eff }}$ ranges from $0.44 \mu \mathrm{m}^{2}$ for the highly confined $\mathrm{TE}_{00}$ mode to a four times larger $A_{\text {eff }}$ of $1.68 \mu \mathrm{m}^{2}$ for the $\mathrm{TM}_{10}$ mode. Note that, although not directly visible from Eq. 2.2, $A_{\text {eff }}$ is wavelength dependent as the mode field distribution is wavelength dependent. A further consequence of the varying confinement of the modes is their different effective refractive indices, $n_{\text {eff. }}$. Since more of the electric field is located in the lower index $\mathrm{SiO}_{2}$ cladding, $n_{\text {eff }}$ is lowered for the higher-order modes. In the given example, the value for $n_{\text {eff }}$ ranges from 1.66 for the $\mathrm{TE}_{00}$ mode to 1.46 for the $\mathrm{TM}_{10}$ mode, the latter being very close to the refractive index of the $\mathrm{SiO}_{2}$ cladding.

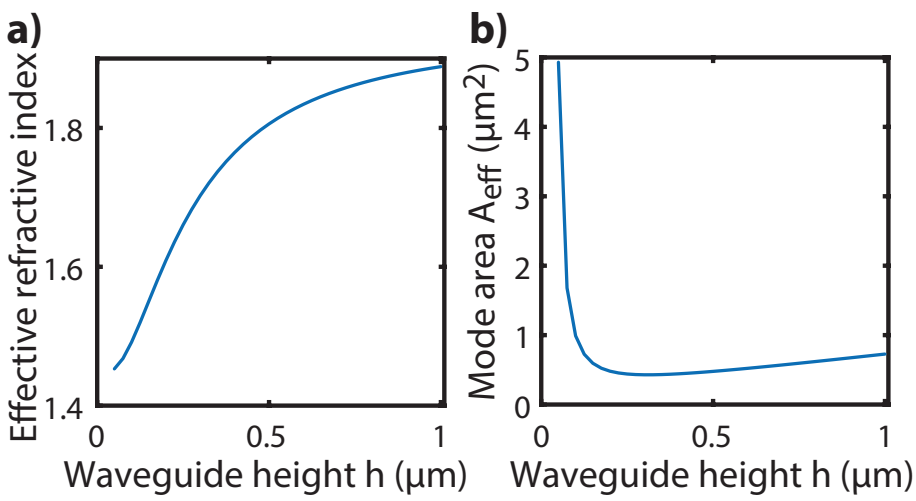

Figure 2.2: The effective refractive index, $n_{\text {eff, }}$ (a) and the effective mode area, $A_{\text {eff, }}$ (b) as a function of $h$ for $w=1 \mu m$, a wavelength of $1064 \mathrm{~nm}$, and the fundamental quasi-TE mode.

To investigate the influence of the waveguide geometry on the spatial confinement of the optical mode, we show in Fig. 2.2 calculated $n_{\text {eff }}$ and $A_{\text {eff }}$ for the fundamental quasi-TE mode as a function of $h$, while keeping $w=1.0 \mu \mathrm{m}$ and $\lambda=1064 \mathrm{~nm}$. It can be seen that $n_{\text {eff }}$ increases with increasing $h$ and approximates the refractive index of bulk silicon nitride for large values of $h$ as more and more of the mode is located in the waveguide core. On the other hand, Fig. 2.2(b) shows that $A_{\text {eff }}$ has a local minimum at $0.43 \mu \mathrm{m}^{2}$ for $0.3 \mu \mathrm{m}$, corresponding to maximum confinement. The effective mode area increases slightly when $h$ is increased away from this local minimum and $A_{\text {eff }}$ increases considerably faster when $h$ is decreased, indicating that mode confinement weakens quickly. This expansion is used in inverse tapers, or so-called spot-size converters [5], where the dimension of the waveguide core is decreased to expand the waveguide mode and achieve more efficient coupling with, e.g., optical fibers. 


\subsubsection{Waveguide dispersion}

In this thesis, we focus on broadband wavelength conversion using ultrashort pulses. For example, in Chapter 3 we investigate conversion of a picosecond input laser pulse into a signal and idler pulse with different wavelengths, and calculate their tuning range of more than $700 \mathrm{~nm}$. In Chapter 6 we present supercontinuum generation based on femtosecond input laser pulses and the generated spectrum spans more than $1600 \mathrm{~nm}$. The variation of the refractive index with wavelength, also called (material) dispersion, is very important in understanding the propagation of ultrashort pulses. Dispersion explains, e.g., how pulses broaden as they propagate and why two pulses with different center wavelengths travel at different phase and group velocities in the same medium. Finally, the dispersion plays a crucial role in increasing or reducing the efficiency of nonlinear optical frequency conversion via so-called phase matching, as described in Section 2.2. Whenever numeric examples are given, the materials $\mathrm{Si}_{3} \mathrm{~N}_{4}$ and $\mathrm{SiO}_{2}$, which form the waveguides used in this thesis, will be used.
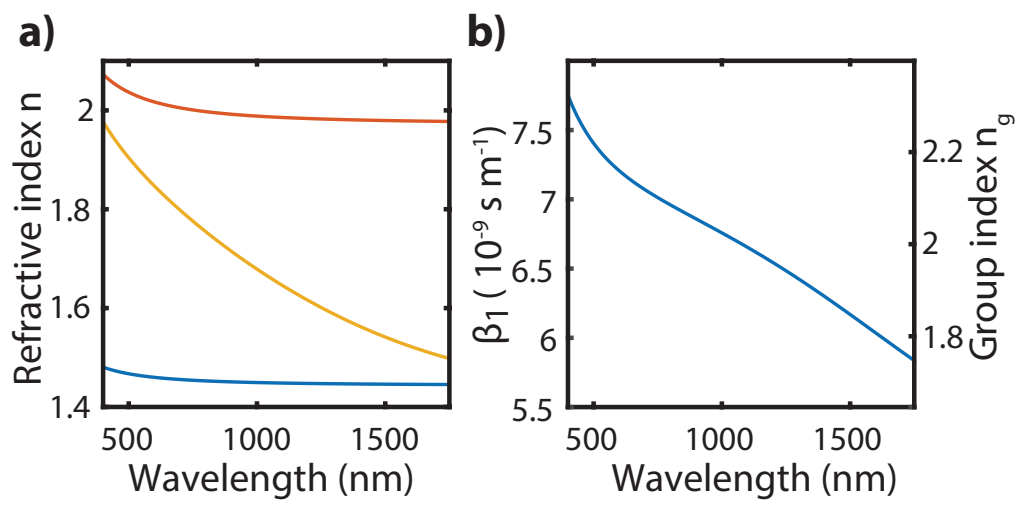

Figure 2.3: (a) Refractive index as a function of wavelength for the fundamental quasi-TE mode of a silicon nitride waveguide (yellow) with $w=1.0 \mu \mathrm{m}$ and $h=0.25 \mu \mathrm{m}$ and bulk $\mathrm{Si}_{3} \mathrm{~N}_{4}$ (red) and $\mathrm{SiO}_{2}$ (blue). (b) Derived first order of dispersion, $\beta_{1}$, from (a) on the left axis and the corresponding group index on the right axis.

The effective refractive index, $n_{\text {eff }}$, for the fundamental quasi-TE mode as a function of $\lambda$ is shown in Fig. 2.3 for a waveguide with the same dimension as discussed earlier $(w=1.0 \mu \mathrm{m}$ and $h=0.25 \mu \mathrm{m})$. It can be seen that $n_{\text {eff }}$ (yellow curve) is located between the index of bulk $\mathrm{Si}_{3} \mathrm{~N}_{4}$ (red) and $\mathrm{SiO}_{2}$ (blue). The effective refractive index shows a stronger wavelength dependence than either of the two materials separately, because the electric field distribution itself is also wavelength dependent. For short wavelengths, the mode is mainly located inside the waveguide core and, hence, approaches the material dispersion of $\mathrm{Si}_{3} \mathrm{~N}_{4}$. At longer wavelengths, the mode is mainly located in the cladding and approaches the dispersion of $\mathrm{SiO}_{2}$. This shows that, by choosing the dimension 
of the waveguide core, it is possible to engineer the resulting dispersion of the waveguide to match particular applications.

The dependence of the propagation constant $\beta$ on the refractive index, $n(\omega)$, and the angular frequency of light, $\omega$, can be mathematically expressed as a Taylor series [4]:

$$
\beta(\omega)=n(\omega) \frac{\omega}{c}=\beta_{0}+\beta_{1}\left(\omega-\omega_{0}\right)+\frac{1}{2} \beta_{2}\left(\omega-\omega_{0}\right)^{2}+\frac{1}{6} \beta_{3}\left(\omega-\omega_{0}\right)^{3}+\ldots,
$$

where $\beta_{m}$ is the $m$-th order of dispersion given by $\beta_{m}=\left(d^{m} \beta / d \omega^{m}\right)_{\omega=\omega_{0}}$.

The various orders account for different propagation effects, but especially the first two orders of dispersion, $\beta_{1}$ and $\beta_{2}$, are of importance. $\beta_{1}$ is related to the so-called group velocity, $v_{g}$, and the group index, $n_{g}$, of the medium by [4]:

$$
\beta_{1}=\frac{1}{v_{g}}=\frac{n_{g}}{c}=\frac{1}{c}\left(n+\omega \frac{d n}{d \omega}\right) .
$$

The calculated $\beta_{1}$ and $n_{g}$ for the waveguide of Fig. 2.3(a) are shown in Fig. 2.3(b). It can be seen that not only $n_{\text {eff }}$ of the mode changes, but also $n_{g}$. This is important when, e.g., two pulses with different center wavelengths are injected into the same waveguide. The interaction length, i.e., the distance over which two pulses overlap can be calculated by using the difference in $v_{g}$, which is referred to as group velocity mismatch. Another example are microresonators, where the free spectral range, the wavelength distance between two resonances, depends on $n_{g}[6]$. Especially, over broad bandwidths it is important to consider the wavelength dependence of $n_{g}$.

The parameter $\beta_{2}=\frac{d \beta_{1}}{d \omega}$ is called group velocity dispersion (GVD) and represents the temporal broadening of pulses propagating through the waveguides. From the GVD, the dispersion parameter, $D$, can be calculated [4]:

$$
\begin{gathered}
\beta_{2}=\frac{1}{c}\left(2 \frac{d n}{d \omega}+\omega \frac{d^{2} n}{d \omega^{2}}\right) \\
D=\frac{d \beta_{1}}{d \lambda}=-\frac{2 \pi c}{\lambda^{2}} \beta_{2}=-\frac{\lambda}{c} \frac{d^{2} n}{d \lambda^{2}} .
\end{gathered}
$$

Especially for an ultrashort pulse with a broad bandwidth, the difference in $v_{g}$ for the individual spectral components of the pulse results in a strong temporal broadening of the pulse. For $\beta_{2}>0(D<0)$ the longer wavelength components of the pulse possess a higher $v_{g}$ than the components with a shorter wavelength, and therefore arrive earlier at the end of a given waveguide length. This results in a so-called frequency up-chirp of the pulses. When $\beta_{2}>0$ the dispersion is referred to as normal GVD, because most materials exhibit $\beta_{2}>0$ over much of their transparency range. If $\beta_{2}<0(D>0)$, the GVD is called anomalous. Consequently, an anomalous GVD results in the shorter wavelength components of a pulse having a higher $v_{g}$ than its longer wavelength 
components which leads to a temporal broadening of the pulse with the short wavelengths in front, resulting in a so-called down-chirp. The wavelengths where $\beta_{2}$ is zero and the light does not experiences any second-order dispersion are referred to as zero dispersion wavelengths (ZDW).
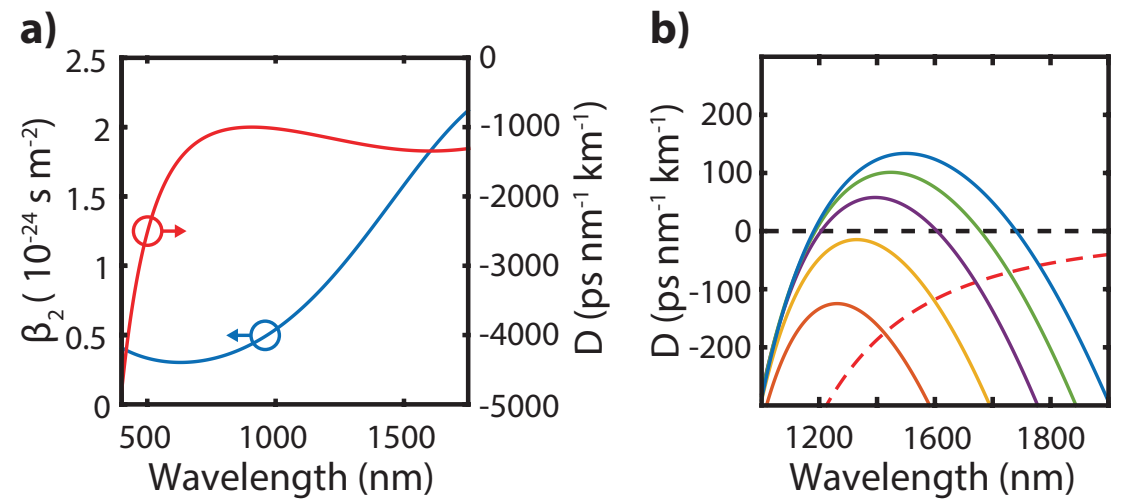

Figure 2.4: (a) Second-order dispersion, $\beta_{2}$, and the corresponding dispersion parameter, $D$, as a function of wavelength for the fundamental quasi-TE mode of a silicon nitride waveguide with $w=1.0 \mu \mathrm{m}$ and $h=0.25 \mu \mathrm{m}$. (b) D of the fundamental quasi-TE mode of silicon nitride waveguides with $w=1.0 \mu \mathrm{m}$ and various $h$ and bulk silicon nitride (red dashed line). h ranges from $0.4 \mu \mathrm{m}$ (orange) in $0.1 \mu \mathrm{m}$ steps to $0.8 \mu \mathrm{m}$ (blue). The waveguides show a varying anomalous dispersion region $(D>0)$ in the near-infrared for $h \geq 0.6 \mu \mathrm{m}$.

In Fig. 2.4(a) $\beta_{2}$ (blue curve) and $D$ (red), calculated from $n_{\text {eff }}$ of Fig. 2.3(a), are shown. It can be seen that the dispersion of the waveguide is normal over the entire calculated wavelength range and that no ZDW is present. To preserve the pulse duration of an ultrashort pulse and to phase match nonlinear processes such as four-wave mixing (see Section 2.2), it is of interest to design waveguides such that they exhibit low and anomalous dispersion. This can be achieved by changing the waveguide dimensions. In Fig. 2.4(b) we show the calculated $D$ as a function of the wavelength for the fundamental quasi-TE mode while varying $h$ from $0.4 \mu \mathrm{m}$ (orange curve) to $0.8 \mu \mathrm{m}$ (blue) in steps of $0.1 \mu \mathrm{m}$ and keeping $w$ fixed at $1.0 \mu \mathrm{m}$. It can be seen that starting from a height of $0.6 \mu \mathrm{m}$ the waveguides exhibit an anomalously dispersive wavelength range in the near-infrared and the width of the anomalous dispersion region increases with increasing $h$. The fabrication and characterization of stoichiometric $\mathrm{Si}_{3} \mathrm{~N}_{4}$ waveguides that exhibit anomalous dispersion over a broad wavelength range is described in Chapter 4. 


\subsection{Nonlinear frequency conversion}

When light propagates through a dielectric material, it induces a polarization, $P$, which is a charge separation that can be quantified as a dipole moment per volume. For light with a low intensity, the response of the dielectric, which is the induced polarization, can be assumed to be linearly dependent from the electric field of the light wave. However, if the intensity is increased the linear material response is no longer a good approximation and the induced polarization $P$ becomes nonlinearly dependent on the field. To describe this mathematically, $P$ is written as [7]:

$$
P=\epsilon_{0}\left(\chi^{(1)} \cdot E+\chi^{(2)} \cdot E E+\chi^{(3)} \cdot E E E+\ldots\right),
$$

where $\epsilon_{0}$ is the vacuum permittivity and $\chi^{(n)}$ is the $n$-th order susceptibility of the material. In its most general form, all susceptibilities are tensors that determine the spatial direction of the polarization vector as induced by multiple field vectors applied simultaneously to the material. The linear susceptibility $\chi^{(1)}$, which is the predominant part of the material response, is in general a complex quantity where the real part of $\chi^{(1)}$ is related to the refractive index $n$ and the imaginary part of $\chi^{(1)}$ is related to the absorption $\alpha_{m}$.

For certain cases, the second-order susceptibility, $\chi^{(2)}$, is the strongest of the nonlinear terms. Nonlinear effects such as second harmonic generation, sum and difference frequency conversion result from $\chi^{(2)}[7]$. However, in order to have a non-vanishing $\chi^{(2)}$ component, the material must not exhibit inversion symmetry [7]. Silicon nitride deposited by chemical vapor deposition (CVD) is considered to be an amorphous material possessing inversion symmetry. Therefore, it is expected that $\mathrm{Si}_{3} \mathrm{~N}_{4}$ would not have a $\chi^{(2)}$. However, recently second harmonic generation has been reported in silicon nitride structures $[8,9]$. Although inversion symmetry is expected to apply to bulk $\mathrm{Si}_{3} \mathrm{~N}_{4}$, this symmetry will be broken at the interface with another material [7]. Alternatively, inversion symmetry may also be broken by stress in the material. Such processes may lead to an effective $\chi^{(2)}$. The precise origin of the $\chi^{(2)}$ nonlinearity of $\mathrm{Si}_{3} \mathrm{~N}_{4}$-based devices is still part of ongoing discussion [9]. The reported strength of the effective $\chi^{(2)}$ in the out-of-plane direction of plasma-enhanced CVD (PECVD) silicon nitride can be rather high, $2.5 \mathrm{pmV}^{-1}$ [9], which is comparable to that of beta barium borate (BBO) [7], a common nonlinear crystal. However, the effective $\chi^{(2)}$ in $\mathrm{Si}_{3} \mathrm{~N}_{4}$ deposited by low-pressure CVD (LPCVD) is estimated to be much smaller, in the range from 0.005 to $0.04 \mathrm{pmV}^{-1}$ [8]. Due to the small values of $\chi^{(2)}$ in LPCVD $\mathrm{Si}_{3} \mathrm{~N}_{4}$, the third-order nonlinearity, $\chi^{(3)}$, is the dominant nonlinearity in our experiments.

The third-order nonlinearity quantifies the strength of various related nonlinear processes such as the Kerr effect, four-wave mixing (see Sec.2.2.1), self-phase modulation (see Sec. 2.2.2), and third harmonic generation, depending on the involved light frequencies and phase-matching conditions. For instance, when all electric fields have the same frequency (frequency degeneracy), 
this results in an intensity dependence of the refractive index, which is known as the quadratic Kerr effect and is usually expressed as [4]:

$$
n(\omega, I)=n_{0}(\omega)+n_{2} \cdot I
$$

where $I$ is the intensity of the electric field, $n_{0}$ is the linear refractive index as described in Section 2.1.3, and $n_{2}$ is the nonlinear index coefficient or so-called Kerr index. The Kerr index is proportional to $\chi^{(3)}$ via [7]:

$$
n_{2}=\frac{3}{4 n_{0}^{2} \epsilon_{0} c} \cdot \chi^{(3)}
$$

The Kerr index, $n_{2}$, for silicon nitride waveguides deposited by CVD is reported to be $2.4 \cdot 10^{-19} \mathrm{~m}^{2} \mathrm{~W}^{-1}$ [10]. When compared to other established waveguide materials, the $n_{2}$ of silicon nitride lies between that of silica (3.2 . $\left.10^{-20} \mathrm{~m}^{2} \mathrm{~W}^{-1}[7]\right)$, as used in optical fibers, and silicon $\left(2.7 \cdot 10^{-18} \mathrm{~m}^{2} \mathrm{~W}^{-1}[7]\right)$, which is used in silicon-on-insulator waveguides.

In optical waveguides, where the mode area, $A_{\text {eff }}$, does not change during the propagation, it is convenient to use the so-called nonlinear parameter, $\gamma$, to express the strength of third-order nonlinear effects [4]:

$$
\gamma=\frac{n_{2} \cdot \omega}{A_{\mathrm{eff}} \cdot c}
$$

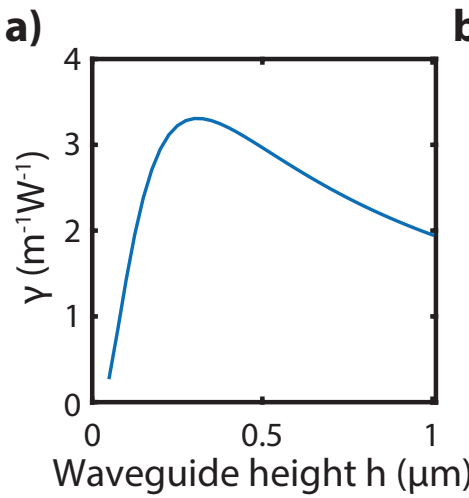

b)

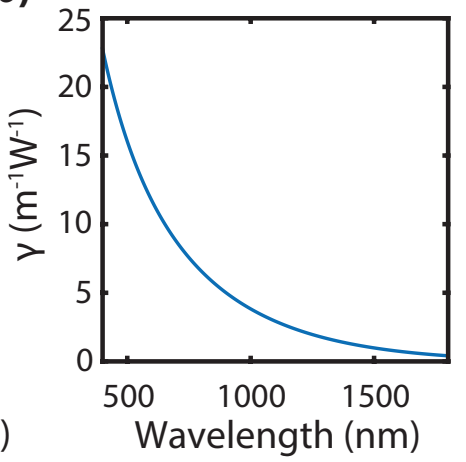

Figure 2.5: (a) Nonlinear parameter, $\gamma$, as a function of $h$ for a fixed $w$ of $1.0 \mu \mathrm{m}$ and $\lambda$ of $1064 \mathrm{~nm}$ as in Fig. 2.2. (b) $\gamma$ as function of wavelength for a waveguide with $w=1.0 \mu \mathrm{m}$ and $h=0.25 \mu \mathrm{m}$.

To demonstrate the dependence of $\gamma$ on the waveguide dimensions, we calculate $\gamma$ as a function of $h$, while keeping $w$ fixed at $1.0 \mu \mathrm{m}$ and for $\lambda=1064 \mathrm{~nm}$ as in Fig. 2.2(a). The results are shown in Fig. 2.5(a) and it can be seen that a maximum for $\gamma$ of $3.3 \mathrm{~m}^{-1} \mathrm{~W}^{-1}$ is reached at the point of highest mode confinement ( $h=0.3 \mu \mathrm{m}$, see Fig. 2.2(b)). However, $\gamma$ is highly wavelength dependent resulting from its direct dependence on $\omega$ as well as the wavelength 
dependence of $A_{\text {eff }}$. The dependence of $\gamma$ as a function of wavelength is shown in Fig. 2.5(b) for fixed waveguide dimensions, $h=1.0 \mu \mathrm{m}$ and $w=0.25 \mu \mathrm{m}$. It can be seen that the value for $\gamma$ shows a strong monotonic decrease with increasing wavelength, from $14.5 \mathrm{~m}^{-1} \mathrm{~W}^{-1}$ at $532 \mathrm{~nm}$ to $0.8 \mathrm{~m}^{-1} \mathrm{~W}^{-1}$ at $1550 \mathrm{~nm}$. Consequently, the wavelength dependence of $\gamma$ has to be taken into account when considering nonlinear effects spanning a broad bandwidth. For comparison to typical values in other materials, a photonic crystal fiber has $\gamma \approx 0.04 \mathrm{~m}^{-1} \mathrm{~W}^{-1}$ at $1064 \mathrm{~nm}$ [11], while a silicon-on-insulator waveguide has a $\gamma \approx 200 \mathrm{~m}^{-1} \mathrm{~W}^{-1}[12]$ at $1550 \mathrm{~nm}$.

It can be seen from Fig. 2.5 that $\gamma$ can be maximized by optimizing the waveguide dimensions (for a specific wavelength). However, to increase the efficiency of nonlinear processes that involve various different frequencies (unlike the Kerr effect), not only a high $\gamma$ is needed, but also phase matching has to be provided. Phase matching is needed to ensure that the interacting waves that are generated interfere constructively along the propagation via a proper setting of the dispersion. In waveguides the dispersion can be engineered by choosing the waveguide dimensions to phase match nonlinear effects such as four-wave mixing.

\subsubsection{Four-wave mixing}

Four-wave mixing (FWM) is a general term for a nonlinear process of third order, where four optical waves interact. In this Section, we consider the socalled degenerate four-wave mixing (FWM), where the energy of two photons from a strong pump wave with the angular frequency, $\omega_{p}$, is transferred to a signal photon with $\omega_{s}$ and an idler photon with $\omega_{i}$, as shown in Fig. 2.6(a). As a convention, the wave with the higher angular frequency, $\omega_{s}$, is called signal and the lower angular frequency wave, $\omega_{i}$, is called idler. In optical fibers FWM has been used to realize tunable light sources, so-called fiber optical parametric oscillators (FOPO) [13]. In Chapter 3, seeded FWM in a $\mathrm{Si}_{3} \mathrm{~N}_{4}$ waveguide is investigated as a tunable light source for spectroscopic application in coherent anti-Stokes Raman scattering.

In degenerate FWM there is energy conservation involving the pump, signal, and idler photons, which can be expressed as:

$$
2 \hbar \omega_{p}-\hbar \omega_{s}-\hbar \omega_{i}=0
$$

where $\hbar$ is the reduced Planck constant. A result of energy conservation is that the differences $\omega_{p}-\omega_{i}$ and $\omega_{s}-\omega_{p}$ are the same and equal to $\Omega$, as indicated in Fig. 2.6(a).

Furthermore, to achieve efficient conversion from the pump wave to signal and idler waves, phase matching has to be fulfilled, such that the effective wavenumber mismatch, $\kappa$, is [4]

$$
\kappa=2 \beta_{p}-\beta_{s}-\beta_{i}+\gamma P=0 .
$$



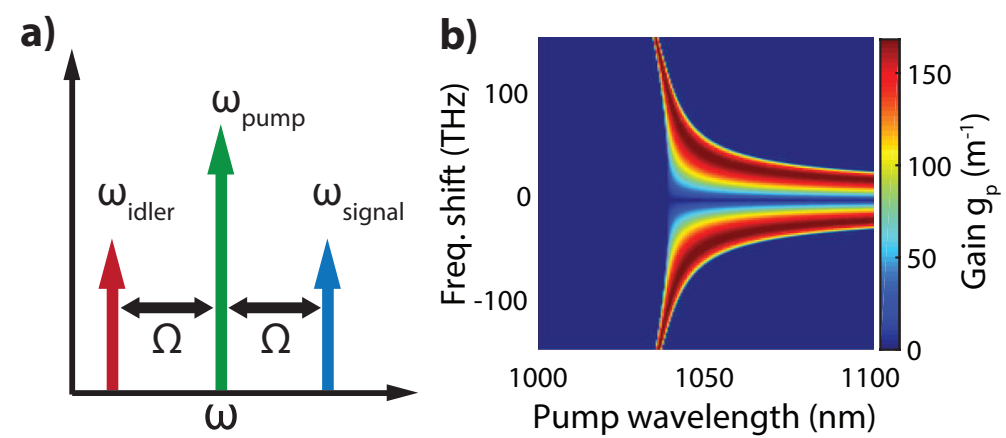

Figure 2.6: (a) Schematic of the degenerate four-wave mixing process. (b) Four-wave mixing gain for the fundamental quasi-TE mode of a silicon nitride waveguide $(w=1.0 \mu \mathrm{m}, h=0.8 \mu \mathrm{m})$ for various wavelengths and frequency shifts.

In this expression $\beta_{p}, \beta_{s}$, and $\beta_{i}$ are the propagation constants of the pump, signal, and idler waves, respectively, and $P$ is the pump power. The effective wavenumber mismatch, $\kappa$, differs from the analogue mismatch equation known from $\chi^{(2)}$ nonlinear processes in that an extra term appears that quantifies the effects of the nonlinear phase-shift due to the Kerr effect, $\gamma P$. Furthermore, since in silicon nitride and silicon dioxide waveguides $\gamma$ is always positive, the sum of the propagation constants has to be negative to fulfill phase matching $(\kappa=0)$ at a nonzero power. If we consider the pump, signal, and idler waves traveling in the same mode, e.g., the fundamental TE mode, phase matching for degenerate FWM can be achieved when the waveguide exhibits anomalous group velocity dispersion $(D>0)$. Alternative approaches for phase matching, as reported for optical fibers can be based on birefringence in the waveguide [14] or higher-order modes [15].

The parametric gain, $g_{p}$, of degenerate FWM as a function of the effective phase-mismatch, $\kappa$, is given by [4]

$$
g_{p}=\sqrt{(\gamma P)^{2}-(\kappa / 2)^{2}} .
$$

Equation 2.13 shows that the shape of the gain can be controlled via choosing a suitable dispersion of the waveguide. Furthermore, the maximum gain is realized for zero phase-mismatch and given by $\gamma \cdot P$, and the bandwidth of the gain is dependent on the pump power. In Fig. 2.6(b) we show the parametric gain calculated as a function of pump wavelength and the frequency shift between pump and signal wave (and idler). Here, a pump power of $100 \mathrm{~W}$, which typically can be obtained from a picosecond laser, was used and a waveguide with $w=1.0 \mu \mathrm{m}$ and $h=0.8 \mu \mathrm{m}$ (as in Fig. 2.4(b)). For this waveguide and assuming the fundamental quasi-TE mode, a ZDW is found at $1039 \mathrm{~nm}$. The wavelength range is chosen to contain this ZDW and both anomalous and normal GVD regimes. It can be seen that the parametric gain broadens in the 
anomalous regime towards the ZDW, while vanishing in the normal dispersion regime. The parametric gain is calculated up to a frequency shift of $150 \mathrm{THz}$ with a pump wavelength of $1040 \mathrm{~nm}$, corresponding to a signal wave at $680 \mathrm{~nm}$ and an idler wave at $2170 \mathrm{~nm}$.

\subsubsection{Self-phase modulation}

The nonlinearity in the refractive index of a material (i.e. the Kerr effect, see Eq. 2.8) produces a change in the phase of an optical pulse, which is proportional to the intensity, when it propagates through that material [4]. This effect is referred to as self-phase modulation (SPM). For an ultrashort optical pulse, the change in the phase with time can be sufficiently large to noticeably broaden the optical spectrum. Considering for simplicity a waveguide with negligible propagation loss and waveguide dispersion, the maximal nonlinear phase-shift, $\phi_{\max }$, of an ultrashort pulse is given by [4]:

$$
\phi_{\max }=\gamma P_{0} L
$$

where $P_{0}$ is the peak power of the optical pulse and $L$ is the propagation distance in the waveguide. SPM results in a change of the initial pulse spectrum as a consequence of a rapidly varying nonlinear phase, $\phi_{n l}(t)$, by the temporal intensity profile of the ultrashort pulse. The spectral change is given by [4]:

$$
\delta \omega(t)=-\frac{\partial \phi_{n l}}{\partial t}=-\phi_{\max } \frac{\partial}{\partial t}|U(0, t)|^{2}
$$

where $U(0, t)$ is the normalized pulse shape at the propagation distance, $\mathrm{z}=0$, which corresponds to the initial pulse shape. From Eq. 2.15, it can be seen that the rising edge of the pulse experiences a red-shift to lower frequencies, while the trailing edge is shifted to higher frequencies. The result is that the pulse acquires a frequency chirp, with red-shifted frequency components in front and blue-shifted frequency components in the back.

To demonstrate the effect of SPM on the spectrum, we calculate the spectral evolution of a pulse propagating through a silicon nitride waveguide (see Fig. 2.7). As an example, we assume a Fourier-limited (unchirped) Gaussian pulse, with a center wavelength of $1064 \mathrm{~nm}$, a pulse duration of $1 \mathrm{ps}$, and $P_{0}=100 \mathrm{~W}$ propagating in a waveguide with $\gamma=3.2 \mathrm{~m}^{-1} \mathrm{~W}^{-1}$ (waveguide dimensions: $w=1.0 \mu \mathrm{m}$ and $w=0.25 \mu \mathrm{m}$ ). The initial spectrum at the entrance of the waveguide (taken as $z=0$ ) is shown in Fig. 2.7(a) as well as the spectrum modified by SPM after $0.5 \mathrm{~cm}$ (b), $1.5 \mathrm{~cm} \mathrm{(c),} \mathrm{and} 2.5 \mathrm{~cm}$ (d) of propagation. It can be seen that the spectrum broadens with increasing $z$ from a Fourier-limited bandwidth of $1.7 \mathrm{~nm}$ to a width of $12 \mathrm{~nm}$ (both FWHM). Aside from broadening, the frequency spectrum attains spectral peaks. This is the result of destructive and constructive interference of the newly created frequency components and is a typical feature in SPM spectra. 
a)
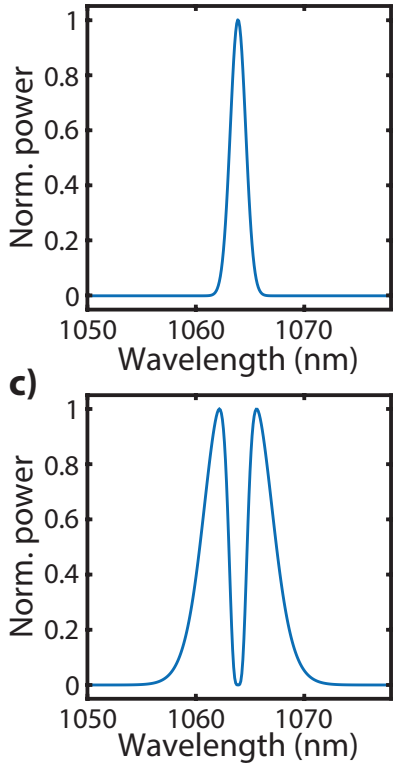

b)
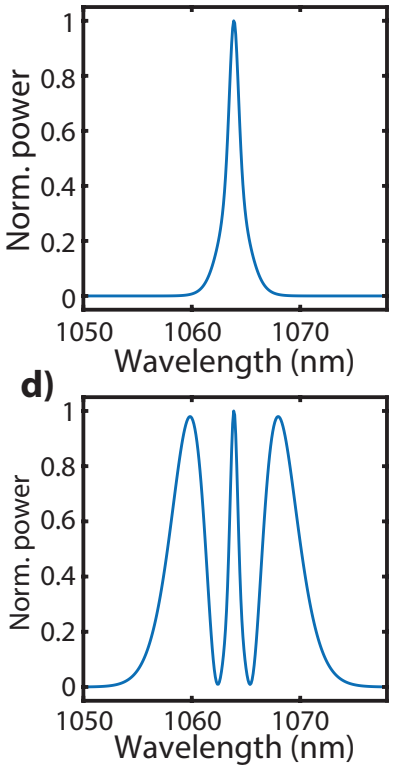

Figure 2.7: The effect of self-phase modulation in a silicon nitride waveguide on the spectrum of an ultrashort pulse with a pulse duration of 1 ps, a center wavelength of $1064 \mathrm{~nm}$, and a peak power of $100 \mathrm{~W}$. The initial pulse spectrum (a) and the spectrum after $0.5 \mathrm{~cm}$ (b), $1.5 \mathrm{~cm}(\mathrm{c})$, and $2.5 \mathrm{~cm}(d)$ of propagation. Note that propagation loss and dispersion are neglected in this calculations. 
In this calculation, we neglected the propagation loss and dispersion of the waveguide for simplicity. However, this is not a realistic approximation, because the propagation loss, $\alpha$, in waveguides (of the order of $0.1-1 \mathrm{~dB} / \mathrm{cm}$ ) is typically high when compared to optical fibers $(\approx 0.1-1 \mathrm{~dB} / \mathrm{km})$. The propagation loss results in a decay of the peak power in the waveguide during propagation, which limits the effective interaction length to typically a few centimeters. Furthermore, the dispersion, i.e., the GVD, in waveguides leads to a temporal broadening of the pulse and, especially, for broad spectra the GVD has to be taken into account. To include dispersion as well as loss in the calculation of nonlinear pulse propagation a more complete model has to be used. In Section 2.2.3 we describe the nonlinear Schrödinger equation to model the nonlinear pulse propagation more comprehensively.

\subsubsection{Nonlinear Schrödinger equation}

As concluded in Section 2.2.2, to model the nonlinear pulse propagation in optical waveguides more realistically, it is necessary to include dispersion and losses, as well as other nonlinear effects, in an extended model. In this thesis, we use the generalized nonlinear Schrödinger equation (GNLSE), which includes the nonlinear parameter, $\gamma$, of the waveguides to model effects such as SPM and FWM. The GNLSE is an extended version of the standard nonlinear Schrödinger equation, the difference being that it takes into account higherorder dispersion, the Raman effect, and self-steepening [16]. The model is commonly used in the field of fiber optics and is used to describe, e.g., broadband supercontinuum generation in photonic crystal fibers [16]. Here, we apply the GNLSE to model seeded four-wave mixing (Chapter 3) and supercontinuum generation (Chapter 5 and 6 ). In our model, propagation is considered to be scalar and unidirectional, which assumes that the light is in a single mode of the waveguide, while for the electric field the slowly varying envelope approximation is applied [17].

The GNLSE can be written as [16]:

$$
\begin{aligned}
\frac{\partial E}{\partial z}= & -\frac{\alpha}{2} E+\sum_{k \geq 2} \frac{i^{k+1}}{k !} \beta_{k} \frac{\partial^{k} E}{\partial t^{k}} \\
& +i \gamma\left(1+i \tau_{s} \frac{\partial}{\partial t}\right)\left(E(z, t) \int_{-\infty}^{+\infty} R\left(t^{\prime}\right)\left|E\left(z, t-t^{\prime}\right)\right|^{2} d t^{\prime}\right) .
\end{aligned}
$$

The first two terms on the right hand side of Eq. 2.16 take into account linear propagation, where $\alpha$ is the power loss during propagation and $\beta_{k}$ is the $k$-th order of dispersion from Eq. 2.3.

The third term on the right-hand side in Eq. 2.16 models third-order nonlinear effects, where $\gamma$ is the nonlinear parameter from Eq. 2.10. To take into account the nonlinearity of the waveguide as a function of wavelength, the time derivative part in Eq. 2.16 is introduced which models nonlinear effects 
such as self-steepening and formation of optical shock fronts [16]. Especially, the wavelength dependence of $\gamma$, e.g., as shown in Fig. 2.5(b), has to be taken into account and is modeled in the GNLSE by the so-called shock term, $\tau_{s}$, which can be expressed as [16, 18]:

$$
\tau_{s}=\frac{1}{\omega_{0}}-\left[\frac{1}{n_{\mathrm{eff}}(\omega)} \frac{d n_{\mathrm{eff}}(\omega)}{d \omega}\right]_{\omega_{0}}-\left[\frac{1}{A_{\mathrm{eff}}(\omega)} \frac{d A_{\mathrm{eff}}(\omega)}{d \omega}\right]_{\omega_{0}},
$$

where $\omega_{0}$ is the center angular frequency of the pulse.

The Raman effect is taken into account by a delay term in the response function, $R(t)$, in Eq. 2.16, is given [16]:

$$
R(t)=\left(1-f_{R}\right) \delta(t)+f_{R} h_{R}(t),
$$

where $\delta(t)$ is the delta distribution, $h_{r}$ is the Raman response function, and $f_{R}$ is the contribution of the delayed Raman response to the instantaneous electronic response of the material. For example, the value $f_{R}$ has been reported to be 0.18 for standard silica fibers [4]. The Raman response function is derived from the Raman shift of the material as described for silica fibers by Stolen et al. [19]. However, for silicon nitride waveguides there is yet insufficient data on the Raman effect to extract exact values for $h_{R}$ and $f_{R}$. Furthermore, it has been reported that the Raman effect does not significantly effect supercontinuum generation in $\mathrm{Si}_{3} \mathrm{~N}_{4}$ waveguides [20]. Therefore, we neglected the Raman effect in most of the calculations in this thesis. Mathematically, to neglect the Raman effect $f_{R}$ is assumed to be zero and $R(t)$ in Eq. 2.18 becomes $\delta(t)$ so the integral in Eq. 2.16 simplifies to $|E(z, t)|^{2}$.

Here, the GNLSE is numerically solved using a split-step Fourier method as reported by Kues et al. [21].

\subsubsection{Supercontinuum generation}

As discussed in Section 2.2.2, SPM continuously changes the spectrum of an intense, ultrashort pulse as it propagates through a waveguide. After sufficiently long propagation the SPM-induced broadening of the spectrum can exceed several hundreds of nanometers and is then often referred to as supercontinuum generation (SCG). The most common way to generate a supercontinuum is to inject intense, ultrashort pulses into a nonlinear medium. The spectral broadening induced by SPM when an ultrashort optical pulse propagates through the nonlinear waveguide is accompanied by a temporal broadening. Consequently, the peak power drops and an effective interaction length can be defined as the length where broadening of the optical spectrum stops due to a too low peak power in the pulse. Therefore, for efficient SCG in waveguides the effective interaction length has to be increased by dispersion engineering. As we saw in Section 2.2.2, SPM results in red-shifted frequencies being generated at the rising edge of the pulse and blue shifted components at the trailing edge of the 
pulse. In a medium with normal group velocity dispersion, the red shifted and blue shifted components would continue to separate in time, because the group velocity is higher for the red components than for the blue. In this case, the temporal broadening induced by SPM is further increased by normal GVD, which results in a quick reduction of peak power and efficient SCG cannot be obtained. On the other hand, if the waveguide is designed to exhibit anomalous dispersion in the relevant spectral range, the blue components of the spectrum travel faster than the red ones. In this case, the temporal broadening of the pulse is reduced, and efficient SCG is possible.

In the anomalous GVD regime, the balance between the temporal broadening by SPM and compression by the dispersion of the waveguide, as described above, can lead to the formation of temporal solitons [16]. These temporal solitons preserve their temporal shape during propagation through the waveguide by balancing linear and nonlinear effects. Furthermore, when using ultrashort pump pulses with a high peak power, typically at pulse durations of $150 \mathrm{fs}$ and shorter, the initial input pulse evolves into a higher-order soliton, which then breaks up into a number of fundamental solitons during propagation. This break up of the higher-order soliton is referred to as soliton fission and is induced by pertubations of the soliton such as higher-order dispersion [16].

So far we have seen that supercontinuum generation requires anomalous dispersion, which is also the case for FWM (see Eq. 2.12). However, phase matching can also be realized for normal GVD within a spectrally narrow region. The waves resulting from this type of phase matching are known as dispersive waves. The generation of dispersive waves is due to phase matching between the dispersive wave and a propagating soliton. The phase matching is realized through higher orders of dispersion, and is therefore only effective over a narrow spectral band. The angular frequency $\omega_{\mathrm{dw}}$ of a dispersive wave, can be analytically predicted by the phase-matching condition [4]:

$$
\beta_{2} \Omega^{2}+\frac{\beta_{3}}{3} \Omega^{3}+\frac{\beta_{4}}{12} \Omega^{4}-\gamma P_{s}=0,
$$

where $\beta_{n}$ is the $n$-th order of dispersion, as described in Eq. 2.3, evaluated at the angular frequency of the soliton, $\omega_{s}, \Omega=\omega_{s}-\omega_{\mathrm{dw}}$ is the frequency difference between the dispersive wave and the soliton, and $P_{s}$ is the peak power of the soliton.

As described above, it is important that the pump wavelength experiences anomalous GVD to achieve broadband supercontinuum generation in silicon nitride waveguides. As can be seen in Fig. 2.4(b) the dispersion of the waveguide strongly depends on the waveguide geometry. By changing the waveguide geometry appropriately, the dispersion can be engineered such that it enables SCG at specific pump wavelengths. Such engineering may be accomplished in various different manners, via modifying the cladding refractive index [20, 22], modifying the core refractive index, by changing the waveguide core geometry, or with any combination of the three. In this thesis, silicon nitride waveguides are dispersion engineered for SCG via the geometry of the waveguide core. 



\section{Bibliography}

[1] J. Bures, Guided Optics, (Wiley-VCH, 2009).

[2] E. A. J. Marcatili, "Dielectric rectangular waveguide and directional coupler for integrated optics," Bell Systems Technical Journal 48(21), 20712102 (1969).

[3] A. B. Fallahkhair, K. S. Li, and T. E. Murphy, "Vector finite difference modesolver for anisotropic dielectric waveguides," J. Lightwave Technol. 26(11), 1423-1431 (2008).

[4] G. Agrawal, Nonlinear Fiber Optics, 4th ed., (Academic, 2007).

[5] K. Wörhoff, R. G. Heideman, A. Leinse, and M. Hoekman, "TriPleX: a versatile dielectric photonic platform," Adv. Opt. Techn. 4(2), 189-207 (2015).

[6] J. Heebner, R. Grover, and T. Ibrahim, Optical Microresonators (Springer, 2012), 1st ed.

[7] R. W. Boyd, Nonlinear Optics, 3rd ed., (Academic, 2008).

[8] J. S. Levy, M. A. Foster, A. L. Gaeta, and M. Lipson, "Harmonic generation in silicon nitride ring resonators," Optics Express 19(12), 1141511421 (2011).

[9] T. Ning, H. Pietarinen, O. Hyvärinen, J. Simonen, G. Genty, and M. Kauranen, "Strong second-harmonic generation in silicon nitride films," Appl. Phys. Lett. 100(16), 161902 (2012).

[10] K. Ikeda, R. E. Saperstein, N. Alic, and Y. Fainman, "Thermal and Kerr nonlinear properties of plasma-deposited silicon nitride/silicon dioxide waveguides," Optics Express 16(17), 12987-94 (2008).

[11] NKT Photonics, "Specifications NL-1050-ZERO-2," (2015), http://www.nktphotonics.com/product/nonlinear-photonic-crystalfibers/

[12] F. Leo, S.-P. Gorza, J. Safioui, P. Kockaert, S. Coen, U. Dave, B. Kuyken, and G. Roelkens, "Dispersive wave emission and supercontinuum generation in a silicon wire waveguide pumped around the $1550 \mathrm{~nm}$ telecommunication wavelength," Optics Lett. 39(12), 3623-6 (2014). 
[13] D. K. Serkland and P. Kumar, "Tunable fiber-optic parametric oscillator," Optics Lett. 24(2), 92-94 (1999).

[14] R. H. Stolen, M. A. Bösch, and C. Lin, "Phase matching in birefringent fibers," Optics Lett. 6(5), 213-215 (1981).

[15] R. Stolen, "Phase-matched-stimulated four-photon mixing in silica-fiber waveguides," IEEE J. Quant. Electron. 11(3), 100-103 (1975).

[16] J. M. Dudley and S. Coen, "Supercontinuum generation in photonic crystal fiber," Reviews of Modern Physics 78(4), 1135-1184 (2006).

[17] R. R. Alfano, The Supercontinuum Laser Source, 2nd ed., (Springer, 2006).

[18] K. J. Blow and D. Wood, "Theoretical description of transient stimulated Raman scattering in optical fibers," IEEE J. Quant. Electron. 25(1), 26652673 (1989).

[19] R. H. Stolen, J. P. Gordon, W. J. Tomlinson, and H. A. Haus, "Raman response function of silica-core fibers," J. Opt. Soc. Am. B 6(6), 1159 (1989).

[20] H. Zhao, B. Kuyken, S. Clemmen, F. Leo, A. Subramanian, A. Dhakal, P. Helin, S. Severi, E. Brainis, G. Roelkens, and R. Baets, "Visible-to-nearinfrared octave spanning supercontinuum generation in a silicon nitride waveguide," Optics Lett. 40(10), 2177 (2015).

[21] M. Kues, N. Brauckmann, T. Walbaum, P. Gross, and C. Fallnich, "Nonlinear dynamics of femtosecond supercontinuum generation with feedback," Optics Express 17(18), 15827-41 (2009).

[22] J. M. Chavez Boggio, D. Bodenmüller, T. Fremberg, R. Haynes, M. M. Roth, R. Eisermann, M. Lisker, L. Zimmermann, and M. Böhm, "Dispersion engineered silicon nitride waveguides by geometrical and refractiveindex optimization," J. Opt. Soc. Am. B 31(11), 2846 (2014). 


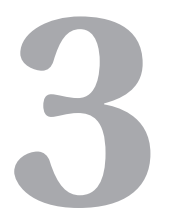

\section{Integrated CARS source based on seeded four-wave mixing in silicon nitride}

We present a theoretical investigation of an integrated nonlinear light source for coherent anti-Stokes Raman scattering (CARS) based on silicon nitride waveguides. Wavelength tunable and temporally synchronized signal and idler pulses are obtained by using seeded fourwave mixing. We find that the calculated input pump power needed for nonlinear wavelength generation is more than one order of magnitude lower than in previously reported approaches based on optical fibers. The tuning range of the wavelength conversion was calculated to be $1418 \mathrm{~nm}$ to $1518 \mathrm{~nm}$ (idler) and $788 \mathrm{~nm}$ to $857 \mathrm{~nm}$ (signal), which corresponds to a coverage of vibrational transitions from $2350 \mathrm{~cm}^{-1}$ to $2810 \mathrm{~cm}^{-1}$. A maximum conversion efficiency of $19.1 \%$ at a peak pump power of $300 \mathrm{~W}$ is predicted. ${ }^{1}$

\footnotetext{
${ }^{1}$ This Chapter has been published as "Integrated CARS source based on seeded four-wave mixing in silicon nitride," Optics Express 21(26), 32123 (2013)
} 


\subsection{Introduction}

Coherent anti-Stokes Raman scattering (CARS) offers the ability to probe both the vibrational frequencies and coherences of materials, making it attractive for many applications. This includes monitoring the temperature and reaction dynamics in combustion engineering [1], remote sensing of explosives [2], standoff infectious agent detection [3], as well as chemically selective imaging [4]. The latter application is particularly challenging since full hyperspectral imaging requires rather specialized light sources. Indeed, the majority of CARS imaging systems aim for only one or two vibrational features, due to the limitations of the sources and signal-to-noise considerations.

The CARS process itself is based on a partially resonant four-wave mixing (FWM) process with at least two input frequencies, whose frequency difference encompasses one or more vibrational transitions. Spectral resolution can then be achieved by a number of means, such as using a broadband excitation pulse and a narrow bandwidth probe pulse [5], temporal delay between frequency chirped excitation and probe [6], and through the use of tunable, narrow bandwidth excitation pulses [7]. The first method relies on light pulses with a broad spectral bandwidth, and selectivity is provided mainly by the spectral bandwidth of the probe pulse. The second method also relies on femtosecond light pulses stretched to a few ps by applying a linear chirp to the spectrum, and the spectral resolution relies on giving the pump and probe equal chirp. The use of tunable narrow bandwidth excitation pulses, on the other hand, allows for better signal-to-noise ratios. This comes, however, at the expense of the complexity of the light sources used to provide excitation and probe pulses. In order to achieve spectral resolution at a relatively high peak power, at least two temporally synchronized pulses in the picosecond regime are preferred, because their spectral bandwidth is in the order of the bandwidth of typical vibrational transitions [4]. Furthermore, the pulses have to be widely tunable, such that their difference frequency covers different vibrational transitions, which lie typically below $3200 \mathrm{~cm}^{-1}$.

Often complex light sources are used, such as optical parametric oscillators [7] or electronically synchronized laser oscillators [8]. Recently, all-fiber approaches have been introduced, which take advantage of the wide natural bandwidth of FWM in optical fibers - especially photonic crystal fibers, pumped near the zero dispersion wavelength. The optical pulse bandwidth can be reduced through filtering [9], temporal focusing [6], and injection seeding [10-12]. A major advantage of all-fiber approaches, compared to lasers and optical parametric oscillators, is their potential compactness and relatively easy maintenance. Another advantage is that temporal synchronization can be achieved by carefully designing the fiber dispersions without the need of an external delay stage [11]. Injection seeding is an attractive option, because it allows control over the emitted signal and idler spectra and increases the spectral power densities needed for CARS applications. Nevertheless, fiber solutions lack compatibility with integrated photonics. This makes it difficult 
to couple CARS light sources with microfluidic devices for biological sensing applications [13], and integrated light sources [14, 15] for ease of tunability.

A source based on integrated photonics is of great interest for analyzing CARS spectra in a lab-on-a-chip setup, which may provide label-free analytical techniques [16]. It is known that efficient wavelength conversion in integrated devices is possible due to high index contrast and using materials with a higher nonlinear response compared to silica based fibers. Indeed, efficient FWM has been shown in highly nonlinear waveguide materials like silicon [17], chalcogenide glasses [18] and doped silica [19]. More recently silicon nitride $\left(\mathrm{Si}_{3} \mathrm{~N}_{4}\right)$, which is also widely used in lab-on-a-chip applications, has been applied for waveguide-based FWM. Silicon nitride waveguides with core thicknesses beyond $500 \mathrm{~nm}$ have become available [20-22]. These increased core thicknesses are sufficient to place the zero dispersion wavelength (ZDW) in the near-infrared, allowing efficient phase-matched FWM. Silicon nitride has a relatively high Kerr nonlinearity [23] and an appropriate transparency range for the near IR wavelengths typically used for CARS. Unlike semiconductor or chalcogenide waveguides, two-photon losses are reduced because the bandgap is relatively large $(5 \mathrm{eV})$ [24]. Finally, linear power losses can be extremely low $(\sim 1 \mathrm{~dB} / \mathrm{cm})$ [25], allowing for relatively long interaction lengths.

Here, we show, through a theoretical investigation, that a silicon nitride waveguide-based CARS light source has many attractive properties. In the normal dispersion region the signal and idler gain spectra become widely spaced, providing a frequency difference between pump and idler that is well-suited to probe the vibrational spectrum of condensed matter samples. By injecting an additional narrow-band, continuous wave seed, with a wavelength that overlaps the signal gain spectrum, a longer-wavelength idler pulse is generated with a narrow bandwidth, instead of the broad bandwidth usually obtained through spontaneous FWM. Furthermore, the required pump laser peak power is found to be one order of magnitude less than that required for fiber based approaches.

\subsection{Integrated CARS source}

We consider a stoichiometric silicon nitride ridge waveguide with a rectangular cross-section deposited on a silica substrate. In order to have maximum conversion efficiency for a frequency difference between pump (taken as $1064 \mathrm{~nm}$ ) and idler in the range below $3200 \mathrm{~cm}^{-1}$, we choose the zero dispersion wavelength to be at $1069 \mathrm{~nm}$. The corresponding waveguide dimensions are then a width of $1630 \mathrm{~nm}$ and a height of $700 \mathrm{~nm}$. Different pump wavelengths can also be accommodated by using different waveguide dimensions, however, all the following calculations are for the given pump wavelength of $1064 \mathrm{~nm}$ as it is readily available in practice. In order to numerically study the nonlinear dynamics of FWM in waveguiding structures, a time-dependent calculation of the light field in ideally three spatial dimensions would be required, however, this is computationally challenging. Instead, we use the one-dimensional gen- 
eralized nonlinear Schrödinger equation [26]. To reduce the problem to one spatial dimension, the nonlinear coefficient $\gamma=n_{2} \omega /\left(c A_{\text {eff }}\right)$ [26] and the dispersion of the waveguide is pre-calculated by solving for the transverse mode profiles of the fundamental modes for the pump, signal and idler fields with a finite-element mode solver. In the expression for the nonlinear coefficient $\gamma, n_{2}$ is the nonlinear refractive index, $\omega$ the pump frequency, $\mathrm{A}_{\text {eff }}$ the effective area of the fundamental quasi-TE mode and $c$ the speed of light. From the mode profile, the nonlinear coefficient was calculated to be $1.93 \mathrm{~m}^{-1} \mathrm{~W}^{-1}$, where $n_{2}=2.4 \times 10^{-15} \mathrm{~cm}^{2} / \mathrm{W}$, is taken from [23], Ikeda et al.

The spectrum of the FWM small-signal gain [26] for the fundamental quasiTE mode was calculated using $\gamma$ and the waveguide dispersion relations obtained from the finite element calculations. The results for waveguide widths from $1610 \mathrm{~nm}$ to $1650 \mathrm{~nm}$ are displayed in Fig. 3.1(a), where a pump wavelength, $\lambda_{p}$, of $1064 \mathrm{~nm}$ and a power, $P_{0}$, of $300 \mathrm{~W}$ have been assumed. The full width at half maximum (FWHM) of the FWM gain spectrum, as in Fig. $3.1(\mathrm{a})$, is $1735 \mathrm{~cm}^{-1}$ at a waveguide width of $1630 \mathrm{~nm}$ (red curve). This significant bandwidth is sufficient to scan over a large range of vibrational levels. Furthermore, Fig. 3.1(a) shows that the peak in the parametric gain can be shifted to a different frequency by changing the waveguide width. This is due to the change in location of the zero dispersion wavelength, and the resulting change in waveguide dispersion determines the region where the FWM process is phase matched. The vibrational frequency spectrum of interest lies at frequencies below $3200 \mathrm{~cm}^{-1}$. The spectral coverage of the FWM in Fig. 3.1(a) is mostly at a higher frequency difference. However, the full nonlinear calculations presented below show that the depletion of the pump results in smaller frequency shifts, which are in the desired range.

The seeded FWM pulse propagation is calculated by numerically integrating the generalized nonlinear Schrödinger equation using a split-step Fourier method as reported in [27], Kues et al. The slowly varying envelope approximation has been applied to the light field, that includes shot noise [28]. To obtain a spectrally narrow CARS Stokes pulse, the idler spectrum is set by injecting a single-frequency, continuous wave at the signal frequency, $\omega_{s}$, with a power, $P_{s}$, of $100 \mathrm{~mW}$. The temporal shape of the pump pulse is Gaussian with a FWHM of $10 \mathrm{ps}$. The peak powers are varied between $150 \mathrm{~W}$ and $350 \mathrm{~W}$, which is in the range of waveguide-based lasers [29]. These parameters result in idler pulses with a bandwidth that is narrower than that typical for vibrational transitions, which are in the range from 10 to $20 \mathrm{~cm}^{-1}$ [4], but still offer the peak powers needed for CARS at feasible average powers.

The propagation of the pulses through the waveguide takes into account nine orders of dispersion from the material and waveguide. The linear power loss of the waveguide is taken as $1 \mathrm{~dB} / \mathrm{cm}$ over the whole wavelength range, as was reported for a silicon nitride ridge waveguide with similar dimensions [21]. The Raman effect is neglected compared to FWM. This is justified for amorphous glasses, as is considered here [26]. 
a)

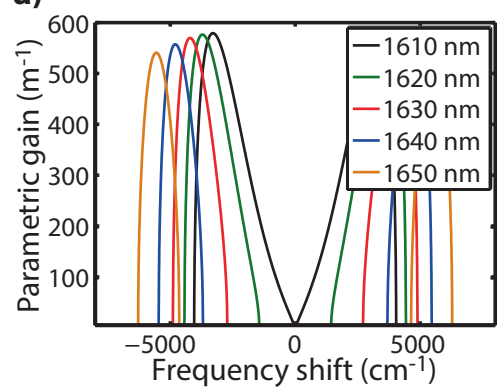

b)

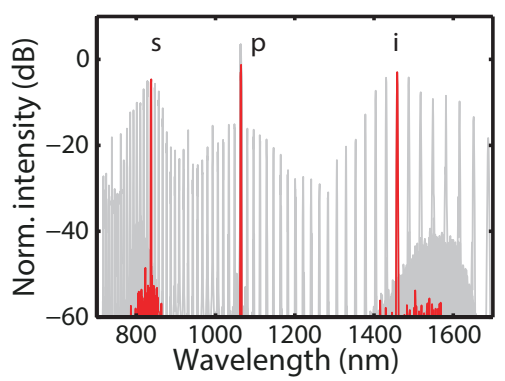

Figure 3.1: (a) Analytically calculated FWM small-signal gain spectra of silicon nitride waveguides with a height of $700 \mathrm{~nm}$ and widths from $1610 \mathrm{~nm}$ to $1650 \mathrm{~nm}$ for a pump power of $300 \mathrm{~W}$. (b) Superimposed spectra of cw seeded FWM after $2 \mathrm{~cm}$ of propagation for a pump pulse with $300 \mathrm{~W}$ peak power, which was numerically calculated using the nonlinear Schrödinger equation. Shown in red is a single spectrum, obtained with a seed wavelength ( $\mathrm{s}$ ) of $828 \mathrm{~nm}$, and resulting in an idler wavelength (i) of $1488 \mathrm{~nm}$, and pumped (p) at $1064 \mathrm{~nm}$. The spectra for a range of seed wavelengths from $714 \mathrm{~nm}$ to $1063 \mathrm{~nm}$ in $4 \mathrm{THz}$ steps are shown in grey.

a)

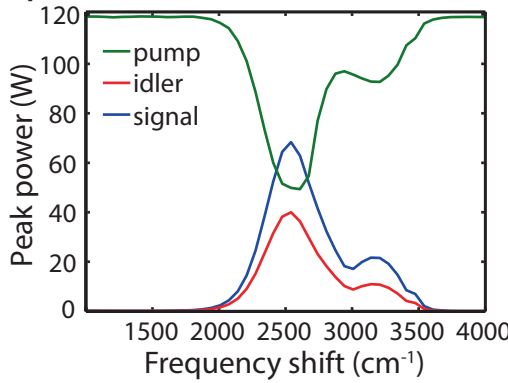

b)

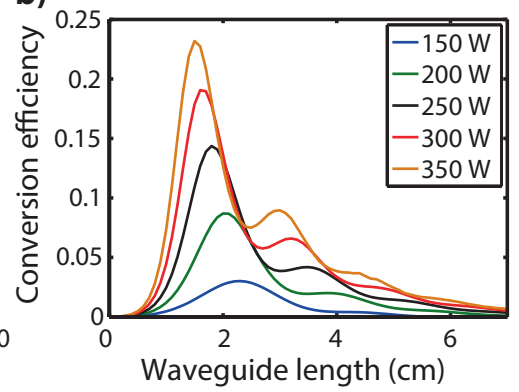

Figure 3.2: (a) Resulting peak powers of pump, signal and idler pulse against the frequency shift from the pump frequency. These results were calculated for a waveguide with a width of $1630 \mathrm{~nm}$, a height of $700 \mathrm{~nm}$, and length of $2 \mathrm{~cm}$. The peak pump power was set to be $300 \mathrm{~W}$. (b) Calculated conversion efficiencies against propagation for peak pump powers from $150 \mathrm{~W}$ to $350 \mathrm{~W}$ seeded at a wavelength of $828 \mathrm{~nm}$. 
In order to show that the injection seeding imposes narrowband signal and idler spectral output tunable over the gain bandwidth, rather than the spontaneous broadband FWM spectrum, the output spectrum was calculated for seed wavelengths in the range of $714-1063 \mathrm{~nm}$ in $4 \mathrm{THz}$ steps, a peak pump power of $300 \mathrm{~W}$ and a waveguide width of $1630 \mathrm{~nm}$ as can be seen in Fig. 3.1(b). It can be seen that FWM occurs over the entire simulated range, however, we require that at least $20 \mathrm{~W}$ of peak power are available for CARS. Using this criteria, it can be seen that both the signal and idler are narrowband, and idler (signal) are generated with the required strength in the wavelength range from 1418 to $1518 \mathrm{~nm}$ (851 down to $819 \mathrm{~nm}$ ). The difference frequency between the pump and idler corresponds to vibrational frequencies in the range of $2346 \mathrm{~cm}^{-1}$ to $2810 \mathrm{~cm}^{-1}$. Comparing these results with Fig. 3.1(a), we observe that the tuning range of $464 \mathrm{~cm}^{-1}$ is not only smaller than the one observed in Fig. 3.1(a) but the range has moved closer to the pump frequency as well. Clearly, the small-signal gain approach overestimates the tuning range and absolute shift of signal and idler frequency with respect to the pump, due to the fact that the calculation of the small-signal gain assumes an undepleted pump [26], while the nonlinear Schrödinger equation includes the full nonlinear dynamics. The peak power of the pump, signal and idler is shown in Fig. 3.2(a) as a function of the frequency shift. This figure shows that maximum peak powers of $68.4 \mathrm{~W}$ and $40.0 \mathrm{~W}$ are obtained for the signal and idler, while the maximum pump depletion results in a minimum peak pump power of $49.4 \mathrm{~W}$.

Moreover, over the tuning range of $2346 \mathrm{~cm}^{-1}$ to $2810 \mathrm{~cm}^{-1}$, the peak power of the idler pulses remains above $20 \mathrm{~W}$, which is large enough to give a strong CARS signal. The calculated seeded idler intensities in this tuning range are at least 25 to $30 \mathrm{~dB}$ higher than the noise level of competing spontaneous FWM (which is broadband). This low background will not contribute significantly to the generated CARS signals.

We calculated the conversion efficiency, defined as $\eta=\left(E_{s}+E_{i}-\right.$ $\left.E_{s, 0}\right) /\left(E_{p, 0}+E_{s, 0}\right)$, where $E_{p, 0}$ is the injected pump energy, and $E_{s, 0}=2$ pJ is the energy injected by the signal seed over the time period of the simulation window $(20 \mathrm{ps}) . E_{s}$ and $E_{i}$ are the pulse energies of the generated signal and idler pulses. The results are shown in Fig. 3.2(b) for five different peak pump powers from $150 \mathrm{~W}$ to $350 \mathrm{~W}$ (with $\lambda_{p}=1064 \mathrm{~nm}, \tau_{p}=10$ ps and $P_{s}=100 \mathrm{~mW}$ ) as a function of waveguide length. The seed wavelength is held constant at $828 \mathrm{~nm}$, which corresponds to an idler wavelength of $1488 \mathrm{~nm}$ and matches the peak of the FWM gain. For pump peak powers of $100 \mathrm{~W}$ or less, no significant FWM was observed.

For peak powers of $150 \mathrm{~W}$ and above the conversion efficiency at first shows strong exponential growth until a maximum is reached. The maximum is due to propagation losses and pump depletion, both of which lower the overall FWM output. After reaching a maximum back conversion starts to set in. For a peak power of $300 \mathrm{~W}$, a maximum conversion efficiency of $19.1 \%$ is reached after $1.8 \mathrm{~cm}$ of propagation. The calculated pulse length of the generated idler pulses is $6.3 \mathrm{ps}$ after a propagation of $1.8 \mathrm{~cm}$ with a spectral width (FWHM) of $1.2 \mathrm{~nm}$, 
which is about two times more than the Fourier limit. The corresponding spectral bandwidth of $5 \mathrm{~cm}^{-1}$ is still smaller than the bandwidths of typical vibrational transitions.

A maximum conversion of $23.2 \%$ was calculated after $1.5 \mathrm{~cm}$ for a peak power of $350 \mathrm{~W}$ and back conversion is observed for longer interaction lengths. This shows that, in order to achieve a maximum conversion efficiency, the length of the waveguide section where FWM takes place has to be carefully selected using calculations that include nonlinear dynamics. Note that the FWM process can easily be stopped by quickly changing the local dispersion of the waveguide, which destroys the phase matching between the waves, by tapering the waveguide, for example.

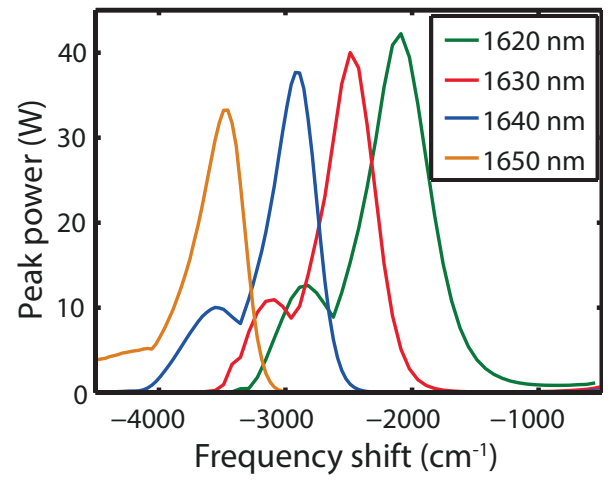

Figure 3.3: Peak power of the idler pulses against the frequency shift for various waveguide widths. The waveguide height is $700 \mathrm{~nm}$, and the interaction length is $2 \mathrm{~cm}$, while the pump peak power is $300 \mathrm{~W}$.

The desired FWM gain spectrum can be controlled by carefully selecting the waveguide dispersion. In order to get to the appropriate dispersion the most obvious parameter to change is the waveguide width since it can be controlled precisely during fabrication of the integrated waveguides. In Fig. 3.3 the calculated peak powers of idler pulses is shown as a function of the frequency shift for waveguide widths ranging from $1610 \mathrm{~nm}$ to $1650 \mathrm{~nm}$ and a height of $700 \mathrm{~nm}$, while pumped with the same parameters as in Fig. 3.2(b). The maximum idler peak power range from $42.3 \mathrm{~W}$ at $2088 \mathrm{~cm}^{-1}\left(\lambda_{i}=1368 \mathrm{~nm}\right)$ at a waveguide width of $1620 \mathrm{~nm}$ to $33.3 \mathrm{~W}$ at $3456 \mathrm{~cm}^{-1}\left(\lambda_{i}=1683 \mathrm{~nm}\right)$ at a width of $1650 \mathrm{~nm}$. The lower conversion efficiency for broader waveguides is explained by the larger effective mode area, $A_{\text {eff }}$, which results in a lower nonlinear coefficient $\gamma$. When comparing the idler peak power of Fig. 3.3 with the small-signal gain of Fig. 3.1(a), it can be seen that the full nonlinear Schrödinger equation predicts the maximum in the idler peak power to occur at a smaller difference frequency than the location of the maximum in the small-signal FWM gain. This can be explained by the power dependence of the FWM gain, since the peak power changes during propagation, due to temporal broadening of the pump pulse, propagation losses, and the high nonlinear conversion efficiency in 
silicon nitride waveguides. This highlights the necessity for numerical studies to predict the exact FWM gain spectrum.

\subsection{Conclusion}

In conclusion, we have shown, through numerical calculations, an efficient way to realise synchronized and tunable picosecond pulses with properties that are highly suitable for application in CARS microscopy and spectroscopy. A high conversion efficiency of $19.1 \%$ is calculated assuming a relatively high power loss of $1 \mathrm{~dB} / \mathrm{cm}$ and a moderate pump peak power of $300 \mathrm{~W}$, which is one order of magnitude lower than in previous reported fiber based approaches. The calculated peak powers as well as the wavelengths of the pump and idler pulse are in the range required for CARS experiments with picosecond pulses. The tuning range of our approach can easily be adjusted by changing the pump wavelength or the waveguide dimensions, because the bandwidth of the FWM gain strongly depends on the dispersion of the waveguides. Furthermore, the spectral coverage can even be doubled by using the signal in place of the pump in a CARS microscope or spectrometer (so the signal becomes the pump for the CARS process), because the signal and idler are of comparable peak power with the pump. These results show that an integrated CARS light source with dimensions on the length scale of $2 \mathrm{~cm}$ can be realized with silicon nitride waveguides at moderate laser powers that are available from waveguide based pulsed and continuous wave laser sources. This approach can easily be adapted for different wavelength ranges and pulse durations as well as for other waveguide platforms. 


\section{Bibliography}

[1] R. J. Hall, and A. C. Eckbreth, "Combustion diagnosis by coherent antiStokes Raman spectroscopy (CARS)," Opt. Eng. 20(4), 494-500 (1981).

[2] A. Portnov, S. Rosenwaks, and I. Bar, "Detection of particles of explosives via backward coherent anti-Stokes Raman spectroscopy," Appl. Phys. Lett. 93, 041115 (2008).

[3] G. Beadie, Z. E. Sariyanni, Y. V. Rostovtsev, T. Opatrny, J. Reintjes, and M. O. Scully, "Towards a FAST CARS anthrax detector: coherence preparation using simultaneous femtosecond laser pulses," Opt. Commun. 244, 423-430 (2005).

[4] C. L. Evans, and X. S. Xie, "Coherent anti-stokes Raman scattering microscopy: chemical imaging for biology and medicine, "Annu. Rev. Anal. Chem. (Palo Alto Calif) 1(1), 883-909 (2008).

[5] T. W. Kee and M. T. Cicerone "Simple approach to one-laser, broadband coherent anti-Stokes Raman scattering microscopy," Optics Lett. 29(23), 2701-2703 (2004).

[6] I. Rocha-Mendoza, W. Langbein, and P. Borri, "Coherent anti-Stokes Raman microspectroscopy using spectral focusing with glass dispersion," Appl. Phys. Lett. 93, 201103 (2008).

[7] M. Jurna, J. P. Korterik, H. L. Offerhaus, and C. Otto, "Noncritical phasematched lithium triborate optical parametric oscillator for high resolution coherent anti-Stokes Raman scattering spectroscopy and microscopy," Appl. Phys. Lett. 89, 251116 (2006).

[8] E. O. Potma, D. J. Jones, J.-X. Cheng, X. S. Xie, and J. Ye, "Highsensitivity coherent anti-Stokes Raman scattering microscopy with two tightly synchronized picosecond lasers, " Optics Lett. 27 13, 1168-1170 (2002).

[9] E. S. Lamb, S. Lefrancois, M. Ji, W. J. Wadsworth, X. S. Xie, and F. W. Wise, "Fiber optical parametric oscillator for coherent anti-Stokes Raman scattering microscopy," Optics Lett. 38(20), 4154-4157 (2013). 
[10] S. Lefrancois, D. Fu, G. R. Holtom, L. Kong, W. J. Wadsworth, P. Schneider, R. Herda, A. Zach, X. S. Xie, and F. W. Wise, "Fiber four-wave mixing source for coherent anti-Stokes Raman scattering microscopy," Optics Lett. 37(10), 1652-1654 (2012).

[11] M. Baumgartl, M. Chemnitz, C. Jauregui, T. Meyer, B. Dietzek, J. Popp, J. Limpert, and A. Tünnermann, "All-fiber laser source for CARS microscopy based on fiber optical parametric frequency conversion," Optics Express 20(4), 4484-4893 (2012).

[12] T. Gottschall, M. Baumgartl, A. Sagnier, J. Rothhardt, C. Jauregui, J. Limpert, and A. Tünnermann, "Fiber-based source for multiplexCARS microscopy based on degenerate four-wave mixing," Optics Express 20(11), 12004-12013 (2012).

[13] A. Ymeti, J. S. Kanger, J. Grevea, G. A. J. Besselink, P. V. Lambeck, R. Wijn, and R. G. Heideman, "Integration of microfluidics with a fourchannel integrated optical Young interferometer immunosensor," Biosens. Bioelectron. 20, 1417-1421 (2005).

[14] R. Oldenbeuving, E.J. Klein, H.L. Offerhaus, C.J. Lee, H. Song, and K.-J. Boller, " $25 \mathrm{kHz}$ narrow spectral bandwidth of a wavelength tunable diode laser with a short waveguide-based external cavity," Laser Phys. Lett. 10, 015804 (2013).

[15] K. Saha, Y. Okawachi, B. Shim, J. S. Levy, R. Salem, A. R. Johnson, M. A. Foster, M. R. E. Lamont, M. Lipson, and A. L. Gaeta, " Modelocking and femtosecond pulse generation in chip-based frequency combs," Optics Express 21(1), 1335-1343 (2013).

[16] C. Camp, S. Yegnanarayanan, A. Eftekhar, H. Sridhar, and A. Adibi, "Multiplex coherent anti-Stokes Raman scattering (MCARS) for chemically sensitive, label-free flow cytometry," Optics Express 17(25), 2287922889 (2009).

[17] M. Foster, A. C. Turner, J. E. Sharping, B. S. Schmidt, M. Lipson, and A. L. Gaeta, "Broad-band optical parametric gain on a silicon photonic chip," Nature 441(7096), 960-963 (2006).

[18] F. Luan, M. D. Pelusi, M. R. E. Lamont, D.-Y. Choi, S. Madden, B. Luther-Davies, and B. J. Eggleton, "Dispersion engineered $\mathrm{As}_{2} \mathrm{~S}_{3}$ planar waveguides for broadband four-wave mixing based wavelength conversion of $40 \mathrm{~Gb} / \mathrm{s}$ signals," Optics Express 17(5), 35414-35420 (2009).

[19] M. Ferrera, L. Razzari, D. Duchesne, R. Morandotti, Z. Yang, M. Liscidini, J.E. Sipe, S. Chu, B. E. Little, and D. J. Moss, "Low-power continuouswave nonlinear optics in doped silica glass integrated waveguide structures," Nat. Photonics 1(12), 737-740 (2008). 
[20] J. S. Levy, A. Gondarenko, M. A. Foster, A. C. Turner-Foster, A. L. Gaeta, and M. Lipson, "CMOS-compatible multiple-wavelength oscillator for on-chip optical interconnects," Nat. Photonics 4(1), 37-40 (2010).

[21] I. Agha, M. Davanço, B. Thurston, and K. Srinivasan, "Low-noise chipbased frequency conversion by four-wave-mixing Bragg scattering in $\operatorname{SiN}_{x}$ waveguides," Optics Lett. 37(14), 2997-2999 (2012).

[22] K. Luke, A. Dutt, C. B. Poitras, and M. Lipson, "Overcoming $\mathrm{Si}_{3} \mathrm{~N}_{4}$ film stress limitations for high quality factor ring resonators," Optics Express 21(19), 22829-22833 (2013).

[23] K. Ikeda, R.E. Saperstein, N. Alic, and Y. Fainman, "Thermal and Kerr nonlinear properties of plasma-deposited silicon nitride/silicon dioxide waveguide," Optics Express 16(17), 12987-12994 (2008).

[24] S.M. Sze, Physics of Semiconductor Devices, 2nd. ed., (Wiley, New York, 1981).

[25] J. F. Bauters, M. J. R. Heck, D. John, D. Dai, M.-C. Tien, J. S. Barton, A. Leinse, R. G. Heideman, D. J. Blumenthal, and J. E. Bowers, "Ultra-lowloss high-aspect-ratio $\mathrm{Si}_{3} \mathrm{~N}_{4}$ waveguides," Optics Express 19(4), 3163-3174 (2011).

[26] G. P. Agrawal, Nonlinear Fiber Optics, 4th ed., (Academic, 2007).

[27] M. Kues, N. Brauckmann, T. Walbaum, P. Gross, and C. Fallnich, "Nonlinear dynamics of femtosecond supercontinuum generation with feedback," Optics Express 17(18), 15827-15841 (2009).

[28] G. Genty, S. Coen, and J. M. Dudley, "Fiber supercontinuum sources," J. Opt. Soc. Am. B. 24, 1771- 1785 (2007).

[29] L. Xiao, X. Cheng, and J. Xu, "High-power Nd:YAG planar waveguide laser with $\mathrm{YAG}$ and $\mathrm{Al}_{2} \mathrm{O}_{3}$ claddings," Opt. Commun. 281, 3781-3785 (2008). 



\section{4 \\ High confinement, high yield $\mathrm{Si}_{3} \mathrm{~N}_{4}$ waveguides for nonlinear optical applications}

In this paper we present a novel fabrication technique for silicon nitride $\left(\mathrm{Si}_{3} \mathrm{~N}_{4}\right)$ waveguides with a thickness of up to $900 \mathrm{~nm}$, which are suitable for nonlinear optical applications. The fabrication method is based on etching trenches in thermally oxidized silicon and filling the trenches with $\mathrm{Si}_{3} \mathrm{~N}_{4}$. Using this technique no stress-induced cracks in the $\mathrm{Si}_{3} \mathrm{~N}_{4}$ layer were observed resulting in a high yield of devices on the wafer. The propagation losses of the obtained waveguides were measured to be as low as $0.4 \mathrm{~dB} / \mathrm{cm}$ at a wavelength of around 1550 nm. $^{1}$

\footnotetext{
${ }^{1}$ This Chapter has been published as "High confinement, high yield $\mathrm{Si}_{3} \mathrm{~N}_{4}$ waveguides for nonlinear optical applications," Optics Express 23(2), 642 (2015).
} 


\subsection{Introduction}

Silicon nitride-based waveguides form a promising, CMOS-compatible platform in integrated photonics research [1]. Especially, stoichiometric silicon nitride $\left(\mathrm{Si}_{3} \mathrm{~N}_{4}\right)$ deposited using low-pressure chemical vapor deposition (LPCVD) offers extremely low intrinsic losses and superior reproducibility. Further advantages of this platform are a broad transparency ranging from the visible [2] to the mid-infrared, a high index contrast and absence of two-photon absorption in the near-infrared, including all the telecommunication bands. Due to their low propagation losses in the C-band as well as their low coupling losses by using spot-size converters $\mathrm{Si}_{3} \mathrm{~N}_{4}$ waveguides are favored in the field of microwave photonics [3, 4] and for novel types (glass-semiconductor) lasers with record low spectral bandwidth $[5,6]$. Furthermore, ultra-low propagation losses in the C-band of $0.32 \mathrm{~dB} / \mathrm{m}$ have been demonstrated with weakly confined modes using $40 \mathrm{~nm}$ thin $\mathrm{Si}_{3} \mathrm{~N}_{4}$ waveguides $[7,8]$. The low confinement of the mode, however, has the drawback of increased bending losses and, as a consequence, limit the density of devices in integrated circuits. Further applications of $\mathrm{Si}_{3} \mathrm{~N}_{4}$ waveguides are, e.g., bio-chemical applications such as optical coherence tomography [9] and lab-on-a-chip devices due to the compatibility of $\mathrm{Si}_{3} \mathrm{~N}_{4}$ waveguides with microfluidic channels $[10,11]$.

Furthermore, $\mathrm{Si}_{3} \mathrm{~N}_{4}$ waveguides are of high interest for nonlinear integrated photonics [1] due to their high Kerr index [12], while supporting highly confined modes due to their high index contrast and lacking nonlinear losses in the near-infrared. Nonlinear effects such as supercontinuum generation [13] and parametric frequency comb generation [14] have been demonstrated. For the latter, huge potential in nonlinear optical signal processing was shown by transmitting a data stream of $1.44 \mathrm{Tbit} / \mathrm{s}$ [15]. It is desirable to select the waveguides such that the pump wavelength for broadband wavelength conversion lies in the spectral region where laser sources are readily available, i.e. 1000 to $2000 \mathrm{~nm}$. However, to obtain phase matching for broadband wavelength conversion, the dispersion of the waveguide must be engineered such that pump wavelength is in the anomalous dispersion regime, while also being close to the zero dispersion wavelength (ZDW).

To achieve high modal confinement and to shift the ZDW to the nearinfrared, the thickness of the silicon nitride waveguide core must be increased substantially from the values that are typically used $(<200 \mathrm{~nm})$. To obtain the dispersion of the waveguides we simulated the effective refractive index $n$ for various wavelengths using a finite element solver (COMSOL Multiphysics) that takes both the dispersion of the materials and, as well, the dispersion induced by the geometry of the waveguide core into account. From this the dispersion parameter, $D=-\frac{\lambda}{c} \cdot \frac{d^{2} n}{d \lambda^{2}}[16]$, can be calculated, where $\lambda$ is the vacuum wavelength of the light and $c$ the speed of light. This is shown in Fig.4.1, where we plot $D$ as a function of the wavelength for waveguides with a fixed width $w=0.8 \mu \mathrm{m}$ and three different heights ranging from 0.8 to $1.2 \mu \mathrm{m}$. As a comparison, the dispersion parameter for bulk $\mathrm{Si}_{3} \mathrm{~N}_{4}$ is shown as well. 


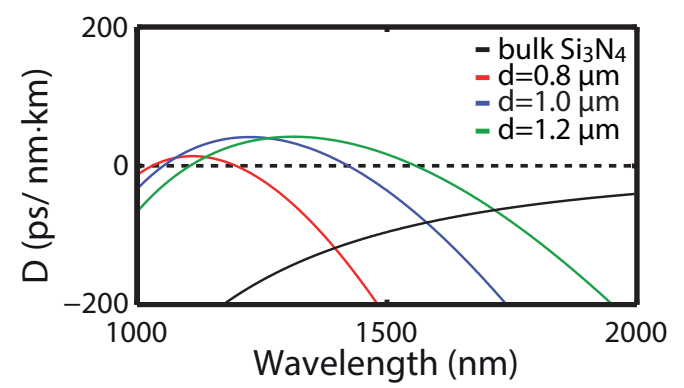

Figure 4.1: Calculated dispersion parameter, D, for bulk $\mathrm{Si}_{3} \mathrm{~N}_{4}$ (black) and for quasi-TM modes (primarily polarized along the $y$ direction) of waveguides with a fixed width of $0.8 \mu \mathrm{m}$ and a height of $0.8 \mu \mathrm{m}$ (red), $1.0 \mu \mathrm{m}$ (blue), and 1.2 $\mu \mathrm{m}$ (green). While bulk $\mathrm{Si}_{3} \mathrm{~N}_{4}$ does not exhibit a $Z \mathrm{DW}$ at wavelengths between $1000 \mathrm{~nm}$ and $2000 \mathrm{~nm}$, the $Z D W$ of the waveguides are $1200 \mathrm{~nm}(d=0.8 \mu \mathrm{m})$, $1420 \mathrm{~nm}(d=1.0 \mu \mathrm{m})$, and $1560 \mathrm{~nm}(d=1.2 \mu \mathrm{m})$.

While bulk $\mathrm{Si}_{3} \mathrm{~N}_{4}$ does not have a ZDW in the wavelength range from 1000 to $2000 \mathrm{~nm}$, the ZDW for the waveguides is calculated to range from $1200 \mathrm{~nm}$ ( $d=0.8 \mu \mathrm{m}$, red) to $1560 \mathrm{~nm}(d=1.2 \mu \mathrm{m}$, green $)$ by changing the thickness $d$ and, hence, providing phase matching for wavelengths around $1550 \mathrm{~nm}$.

However, realizing $\mathrm{Si}_{3} \mathrm{~N}_{4}$ waveguides with sufficient thickness is problematic. Typically, a $\mathrm{Si}_{3} \mathrm{~N}_{4}$ layer is deposited on a thermally oxidized silicon substrate via LPCVD, and waveguide cores are etched from this $\mathrm{Si}_{3} \mathrm{~N}_{4}$ layer. This layer has a limited thickness due to the increasing tensile stress that develops during the deposition of the $\mathrm{Si}_{3} \mathrm{~N}_{4}$ with increasing layer thickness. Ultimately, this stress leads to the formation of cracks when the thickness is larger than about $400 \mathrm{~nm}$ [17]. Recently, improved methods for manufacturing low-loss thick $\mathrm{Si}_{3} \mathrm{~N}_{4}$ waveguides have been introduced [17, 18], however, they still suffer from cracks in the $\mathrm{Si}_{3} \mathrm{~N}_{4}$ layer [17], limiting the yield and reproducibility of device fabrication.

In this paper we report on the fabrication of the first silicon nitride waveguides with thicknesses greater than $800 \mathrm{~nm}$ in a reproducibly crack-free manner. Instead of depositing a thick layer of $\mathrm{Si}_{3} \mathrm{~N}_{4}$, followed by etching the waveguide cores from this layer, we first etch trenches in the silicon dioxide cladding that is formed by thermally oxidizing the top surface of the silicon substrate. The trenches are then filled with $\mathrm{Si}_{3} \mathrm{~N}_{4}$ using LPCVD and the resulting waveguide cores are crack-free for depths of up to $d=1.2 \mu \mathrm{m}$. Note, that this method, in general, provides waveguides with an aspect ratio, $w / d>1$, unlike commonly used $\mathrm{Si}_{3} \mathrm{~N}_{4}$ waveguides. As a result, the width of the waveguide core is the critical parameter that controls stress-induced cracks, which is related to the thickness of the $\mathrm{Si}_{3} \mathrm{~N}_{4}$ layers. Waveguides fabricated in this way are referred to as TriPleX ${ }^{T M}[19,20]$. Details of this process are given in Section 4.2. We then discuss in Section 4.3 the characterization of several thick waveguides that 
a)

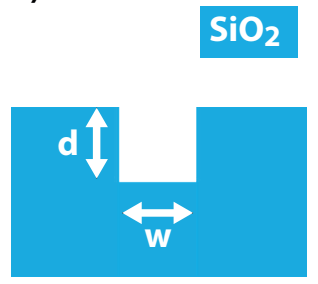

c)

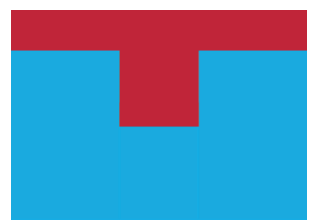

b)

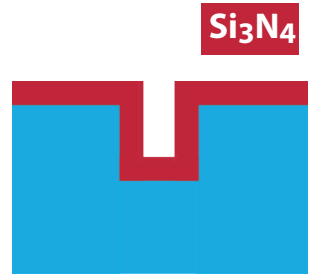

d)

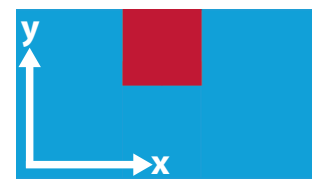

Figure 4.2: Steps of waveguide manufacturing. (a): A trench with depth $d$ and width $w$ is etched into an $8 \mu \mathrm{m}$ layer of thermally oxidized silicon ( $\mathrm{SiO}_{2}$, blue) on a silicon wafer. (b): $250 \mathrm{~nm}$ of $\mathrm{Si}_{3} \mathrm{~N}_{4}$ (red) is deposited homogeneously over all exposed surfaces using LPCVD. (c): The trench is completely filled with $\mathrm{Si}_{3} \mathrm{~N}_{4}$ and a homogeneous $\mathrm{Si}_{3} \mathrm{~N}_{4}$ layer over the whole trench width of up to $0.9 \mu \mathrm{m}$ is formed. (d): The residual $\mathrm{Si}_{3} \mathrm{~N}_{4}$ on the top surface of the wafer is removed from the wafer using chemical-assisted mechanical polishing and dry etching. The remaining $\mathrm{Si}_{3} \mathrm{~N}_{4}$ forms the waveguide core with the dimensions of the etched trench. A top cladding of $\mathrm{SiO}_{2}$ can be deposited optionally on top of the waveguide cores.

are manufactured according to this novel procedure and end with a discussion and conclusion in Section 4.4.

\subsection{Fabrication}

In this method, the waveguide devices are manufactured by filling predefined trenches with $\mathrm{Si}_{3} \mathrm{~N}_{4}$ using LPCVD. The individual fabrication steps are shown in Fig. 4.2. In the first step, as shown in Fig. 4.2(a), reactive ion etching (RIE) is used to etch trenches with a width $w$ and a depth $d$ into the thermally oxidized top surface (oxide depth $8 \mu \mathrm{m}$ ) of a silicon wafer.

In the next step, a $250 \mathrm{~nm}$ thick layer of stoichiometric $\mathrm{Si}_{3} \mathrm{~N}_{4}$ is deposited. During this deposition process, the $\mathrm{Si}_{3} \mathrm{~N}_{4}$ layer grows equally thick from the sidewalls and bottom of the trenches, as illustrated in Fig. 4.2(b). To release stress from the material, the deposition is performed in steps and wafers are allowed to cool down to room temperature between depositions [18]. As the deposition continues, the trench fills with $\mathrm{Si}_{3} \mathrm{~N}_{4}$ until the two layers at the side 
walls merge in the middle of the trench, such that the trench is completely filled, as shown in Fig. 4.2(c). For the manufactured waveguides no more than two deposition steps were required. The final waveguide core is obtained by removing the remaining $\mathrm{Si}_{3} \mathrm{~N}_{4}$ from the top of the wafer using chemical-assisted mechanical polishing and dry etching, as shown in Fig. 4.2(d). To ensure that etching is stopped when the $\mathrm{Si}_{3} \mathrm{~N}_{4}$ from the top of the wafer is removed an etch rate monitor is used. If required, this last processing step can also be applied between deposition steps, to thin the $\mathrm{Si}_{3} \mathrm{~N}_{4}$ layer on top of the wafer to prevent cracks. To complete the waveguide cladding, first a layer of tetraethyl orthosilicate $\left(\mathrm{Si}\left(\mathrm{OC}_{2} \mathrm{H}_{5}\right)_{4}\right.$, TEOS) is deposited by LPCVD, which will form $\mathrm{SiO}_{2}$ after annealing. Second, a layer of $\mathrm{SiO}_{2}$ is grown to a thickness of $8 \mu \mathrm{m}$ by plasma-enhanced chemical vapor deposition (PECVD) (both not shown in Fig. 4.2). To convert the TEOS layer into $\mathrm{SiO}_{2}$ and to reduce the absorption in the near infra-red caused by dangling $\mathrm{Si}-\mathrm{H}$ bonds, the wafers are annealed after deposition at $1150{ }^{\circ} \mathrm{C}$.

Using this procedure, several TriPleX-based $\mathrm{Si}_{3} \mathrm{~N}_{4}$ waveguides were manufactured with their width, $w$, ranging from 0.6 to $0.9 \mu \mathrm{m}$, and depths, $d$, of $0.8,1.0$, and $1.2 \mu \mathrm{m}$. To ensure that these waveguides have low propagation losses, it is important that the trenches have a low surface roughness at the bottom as well as at the side walls, since rougher surfaces will result in higher scattering losses for propagating light. A scanning electron microscope (SEM) picture of an etched trench is shown in Fig. 4.3(a) for a width of $w=0.8 \mu \mathrm{m}$ and a depth of $d=1.0 \mu \mathrm{m}$. Note that the vertical direction of all the SEM pictures in Fig. 4.3 appears to be smaller because the pictures were taken under an angle. Figure 4.3(a) also shows a slight positive taper, meaning that the width of the trench increases from bottom to top, which ensures a conformal deposition. Without tapering, the deposition is not fully conformal, and a gap between the silicon nitride and the trench wall appears, which increases the propagation losses as a consequence.
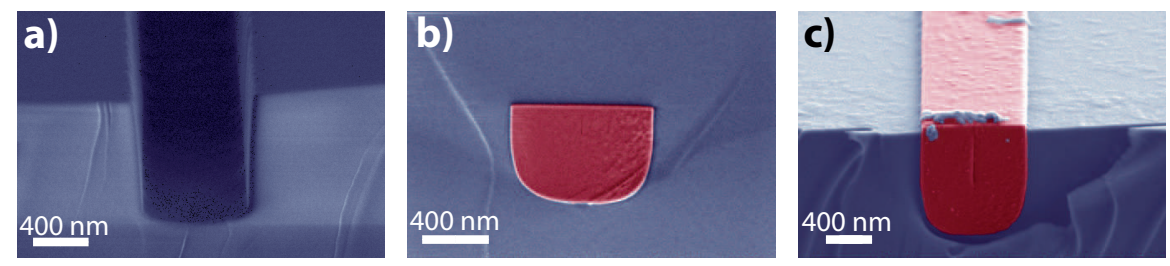

Figure 4.3: SEM pictures (false color): (a) A trench etched in thermally oxidized silicon with $w=0.8 \mu \mathrm{m}$ and $d=1.0 \mu \mathrm{m}$. (b) and (c): the cross section of silicon nitride waveguide core with $w=$ $0.83 \mu m$ and $d=0.8 \mu m$ with a layer PECVD silica on top (b) and $w=0.9 \mu \mathrm{m}$ and $d=1.2 \mu \mathrm{m}$ without a top layer (c). Note that all pictures are taken at an angle, making the vertical direction appear smaller. 
SEM pictures of two completed waveguide cores are shown in Fig. 4.3(b) and $4.3(\mathrm{c})$ with a cross section of $w=0.83 \mu \mathrm{m}$ by $d=0.8 \mu \mathrm{m}$ and $w=0.9 \mu \mathrm{m}$ by $d=1.2 \mu \mathrm{m}$, respectively, with and without a top cladding, respectively. To increase the contrast between the $\mathrm{Si}_{3} \mathrm{~N}_{4}$ and the $\mathrm{SiO}_{2}$ in the SEM pictures, the samples were etched, which resulted in the $\mathrm{Si}_{3} \mathrm{~N}_{4}$ waveguide core sticking out from the $\mathrm{SiO}_{2}$ because of a difference in etching rates of the materials. In Fig. 4.3(b) it can be seen that a homogeneous waveguide core with $w=0.83 \mu \mathrm{m}$ and $d=0.8 \mu \mathrm{m}$ is formed using this manufacturing method.

By comparing the shape of the trench as shown in Fig. 4.3(a) and the waveguides in Figs. 4.3(b) and 4.3(c), the stress induced by the deposition of silicon nitride is evident. The large difference in material stress between the $\mathrm{SiO}_{2}$ and $\mathrm{Si}_{3} \mathrm{~N}_{4}$ layers leads to a change in both the shape of the waveguide as well as an inwards bend of the side walls. The rounded shape of the waveguides leads to a small $(\sim 10 \mathrm{~nm})$ blue-shift in the ZDW compared to the rectangular shape assumed in Fig. 4.1. However, the variations in waveguide dimensions, given by fabrication tolerances, cause larger shifts. In the case of the deepest etching depth $(d=1.2 \mu \mathrm{m})$, it is observed that the change of the waveguide shape is even stronger. Here, the tapering of the trench was insufficient to compensate for the sidewall bending induced by the tensile stress of the $\mathrm{Si}_{3} \mathrm{~N}_{4}$ layer. This effects the final closure of the trench when the deposited $\mathrm{Si}_{3} \mathrm{~N}_{4}$, which grows from the two side walls, meet in the middle. As a result, the trench is not filled entirely with $\mathrm{Si}_{3} \mathrm{~N}_{4}$ as can be seen in Fig. 4.3(c). In future, we will compensate for this effect by applying a stronger positive taper to the sidewall of the trenches.

During the fabrication, a large number of waveguides and waveguide circuits were obtained with the described technique covering a total of six wafers (100 mm diameter) and not a single crack was observed in the $\mathrm{Si}_{3} \mathrm{~N}_{4}$ layers. This clearly indicates that, using our novel approach, crack-free $\mathrm{Si}_{3} \mathrm{~N}_{4}$ waveguides with a width of up to $0.9 \mu \mathrm{m}$ can be fabricated.

\subsection{Characterization}

The propagation losses are an essential characteristic for integrated waveguides, since they are critical for the design of devices such as micro-resonators. By measuring the transmission of a broadband light source through the waveguides, the propagation losses were determined. The broadband light from a superluminescent diode with a center wavelength of $1560 \mathrm{~nm}$ and a spectral bandwidth at full width at half maximum of $45 \mathrm{~nm}$ was used. The light was injected and collected using lensed fibers ( $2 \mu \mathrm{m}$ spot size at $1550 \mathrm{~nm}$ ), while the input polarization was controlled to excite the quasi-TM mode which is primarily polarized along the y-direction (see Fig. 4.2(d)) of the waveguides using an in-line optical fiber polarization controller. The transmitted power was measured for three etching depths, $0.8 \mu \mathrm{m}, 1.0 \mu \mathrm{m}$, and $1.2 \mu \mathrm{m}$, and for four different propagation lengths, using spiral waveguides with lengths of 
$2.30 \mathrm{~cm}, 3.89 \mathrm{~cm}, 6.90 \mathrm{~cm}$, and $10.48 \mathrm{~cm}$ and of various widths.
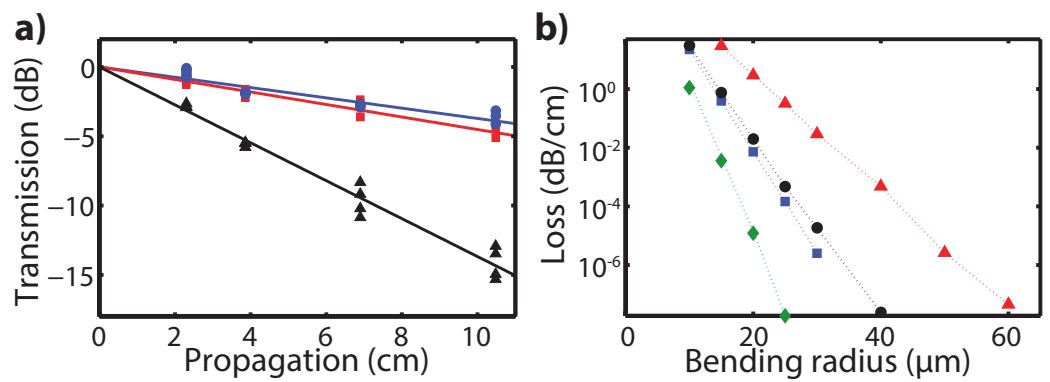

Figure 4.4: (a) Power transmission of light with a center wavelength of $1560 \mathrm{~nm}$ (TM polarization) measured for different waveguide lengths (2.30 cm, $3.89 \mathrm{~cm}, 6.90 \mathrm{~cm}$, and $10.48 \mathrm{~cm}$ ) and waveguide depths: $0.8 \mu \mathrm{m}$ (blue circles), $1.0 \mu \mathrm{m}$ (red squares), and 1.2 $\mu \mathrm{m}$ (black triangles). The waveguide width varies between $0.7 \mu \mathrm{m}$ and $0.9 \mu \mathrm{m}$. The graph has been corrected for the coupling losses, which vary between 13 to $15 \mathrm{~dB}$ in total. (b) Simulated bending losses of the quasi-TM mode at a wavelength of $1550 \mathrm{~nm}$ for various bending radii. Simulations were performed for cross sections and depths of $w=0.5 \mu \mathrm{m}$ and $d=0.8 \mu \mathrm{m}$ (red triangles), $w=0.8 \mu \mathrm{m}$ and $d=0.8 \mu \mathrm{m}$ (blue squares), $w=0.5 \mu \mathrm{m}$ and $d=1.2 \mu \mathrm{m}$ (black circles), and $w=0.8 \mu \mathrm{m}$ and $d=1.2 \mu \mathrm{m}$ (green diamonds).

The transmission as a function of waveguide length is shown in Fig. 4.4(a), and from this data, the propagation loss coefficient is determined to be $0.37 \mathrm{~dB} / \mathrm{cm}(d=0.8 \mu \mathrm{m}$, blue $), 0.45 \mathrm{~dB} / \mathrm{cm}(d=1.0 \mu \mathrm{m}$, red $)$, and $1.37 \mathrm{~dB} / \mathrm{cm}$ $(d=1.2 \mu \mathrm{m}$, black), while $w$ ranged from 0.7 to $0.9 \mu \mathrm{m}$. No variation in the propagation loss was observed for the range of waveguide widths investigated. The propagation losses are believed to be mainly due to scattering losses since absorptive losses have been reported to amount to values of below $0.055 \mathrm{~dB} / \mathrm{cm}$ in $\mathrm{Si}_{3} \mathrm{~N}_{4}$ waveguides [18]. We address the comparably high losses for a $d$ of $1.2 \mu \mathrm{m}$ to higher scattering losses resulting from the small gap in the center of the $\mathrm{Si}_{3} \mathrm{~N}_{4}$ core, as shown in Fig. 4.3(c).

Another important performance parameter is the bending loss, as this indicates how densely devices can be packed on a wafer. In our measurements we found that the bending losses are so low that we could not experimentally distinguish these from the differences in coupling efficiencies from waveguide to waveguide. To quantify bending losses for thick $\mathrm{Si}_{3} \mathrm{~N}_{4}$ waveguides, we simulated them using a finite element solver (FieldDesigner, PhoeniX BV). The simulated bending losses for a quasi-TM mode at a wavelength of $1550 \mathrm{~nm}$ are shown in Fig. 4.4(b) as a function of the radius of the bend for waveguide cross sections of $d=0.8 \mu \mathrm{m}$ and $1.2 \mu \mathrm{m}$ and $w=0.5 \mu \mathrm{m}$ and $0.8 \mu \mathrm{m}$, respectively. As a result of the high confinement, the bending losses of the guided mode are lower for larger waveguide cross sections. As shown in Fig. 4.4(b), e.g., the 
losses for a bending radius of $20 \mu \mathrm{m}$ decrease from $2.9 \mathrm{~dB} / \mathrm{cm}$ for the smallest waveguide cross section, $w=0.5 \mu \mathrm{m}$ and $d=0.8 \mu \mathrm{m}$ (red), to as low as $1.2 \cdot 10^{-5} \mathrm{~dB} / \mathrm{cm}$ for $w=0.8 \mu \mathrm{m}$ and $d=1.2 \mu \mathrm{m}$ (green). The bending losses are negligible for the bigger cross sections when compared to the propagation losses. The simulated bending loss for waveguides with high modal confinement and $d=w=0.8 \mu \mathrm{m}$ (blue) is only $0.7 \mathrm{~dB} / \mathrm{m}$ for a bending radius of $20 \mu \mathrm{m}$. In comparison, to obtain a similar bending loss from a waveguide with low modal confinement [8], the bending radius must be as large as $2 \mathrm{~mm}$ or more. Consequently, the density of waveguide circuits on a wafer can be increased by orders of magnitude using waveguides with a high confinement, which leads to a superior efficiency in the fabrication of $\mathrm{Si}_{3} \mathrm{~N}_{4}$ waveguides.

\subsection{Conclusion}

In conclusion, we have shown a novel way to fabricate TriPleX-based $\mathrm{Si}_{3} \mathrm{~N}_{4}$ waveguides with a width of up $0.9 \mu \mathrm{m}$, while having a depth of up to $1.2 \mu \mathrm{m}$. This method is promising to achieve a high yield compared to previously reported approaches to fabricate $\mathrm{Si}_{3} \mathrm{~N}_{4}$ waveguides of similar cross-section dimensions. The complete area of six wafers with a diameter of $100 \mathrm{~mm}$ was used to produce waveguide structures, while not a single stress-induced crack appeared during or after fabrication. The propagation losses are measured to lie below $0.4 \mathrm{~dB} / \mathrm{cm}$ for waveguides with a depth of $0.8 \mu \mathrm{m}$, while the bending losses were simulated to be below $0.01 \mathrm{~dB} / \mathrm{cm}$ for a bending radius of $20 \mu \mathrm{m}$, both for a quasi-TM mode at a wavelength of $1550 \mathrm{~nm}$.

The measured coupling efficiency and observed variation in coupling efficiency can be improved by implementing spot-size converters at either end of the waveguide. The spot size converter expands the mode field diameter, which makes the coupling easier and less sensitive for imperfections in the flatness of the end facet. 


\section{Bibliography}

[1] D. J. Moss, R. Morandotti, A. L. Gaeta, and M. Lipson, "New CMOScompatible platforms based on silicon nitride and Hydex for nonlinear optics," Nature Photon. 7(8), 597-607 (2013).

[2] S. Romero-García, F. Merget, F. Zhong, H. Finkelstein, and J. Witzens, "Silicon nitride CMOS-compatible platform for integrated photonics applications at visible wavelengths," Optics Express 21(12), 14036-14046 (2013).

[3] C. G. H. Roeloffzen, L. Zhuang, C. Taddei, A. Leinse, R. G. Heideman, P. W. L. van Dijk, R. M. Oldenbeuving, D. A. I. Marpaung, M. Burla, and K.-J. Boller, "Silicon nitride microwave photonic circuits," Optics Express 21(19), 22937-22961 (2013).

[4] D. Marpaung, B. Morrison, R. Pant, C. Roeloffzen, A. Leinse, M. Hoekman, R. Heideman, and B. J. Eggleton, " $\mathrm{Si}_{3} \mathrm{~N}_{4}$ ring resonator-based microwave photonic notch filter with an ultrahigh peak rejection," Optics Express 21(20), 23286-23294 (2013).

[5] R. M. Oldenbeuving, E. J. Klein, H. L. Offerhaus, C. J. Lee, H. Song, and K.-J. Boller, " $25 \mathrm{kHz}$ narrow spectral bandwidth of a wavelength tunable diode laser with a short waveguide-based external cavity," Laser Phys. Lett. 10(1), 015804 (2013).

[6] Y. Fan, R. M. Oldenbeuving, E. J. Klein, C. J. Lee, H. Song, M. R. H. Khan, H. L. Offerhaus, P. J. M. van der Slot, and K.-J. Boller, "A hybrid semiconductor-glass waveguide laser," Proc. SPIE 9135, 91351B (2014).

[7] D. T. Spencer, J. F. Bauters, M. J. R. Heck, and J. E. Bowers, "Integrated waveguide coupled $\mathrm{Si}_{3} \mathrm{~N}_{4}$ resonators in the ultrahigh-Q regime," Optica $\mathbf{1}(3), 153$ (2014).

[8] J. F. Bauters, M. J. R. Heck, D. John, D. Dai, M.-C. Tien, J. S. Barton, A. Leinse, R. G. Heideman, D. J. Blumenthal, and J. E. Bowers, "Ultralow-loss high-aspect-ratio $\mathrm{Si}_{3} \mathrm{~N}_{4}$ waveguides," Optics Express 19(4), 31633174 (2011). 
[9] G. Yurtsever, B. Považay, A. Alex, B. Zabihian, W. Drexler, and R. Baets, "Photonic integrated Mach-Zehnder interferometer with an on-chip reference arm for optical coherence tomography," Biomed. Opt. Express 5(4), 1050-1061 (2014).

[10] H. Cai and A. W. Poon, "Optical trapping of microparticles using silicon nitride waveguide junctions and tapered-waveguide junctions on an optofluidic chip," Lab Chip 12(19), 3803-3809 (2012).

[11] A. Ymeti, J. S. Kanger, J. Greve, G. A. J. Besselink, P. V. Lambeck, R. Wijn, and R. G. Heideman, "Integration of microfluidics with a fourchannel integrated optical Young interferometer immunosensor," Biosens. Bioelectron. 20(7), 1417-1421 (2005).

[12] K. Ikeda, R. E. Saperstein, N. Alic, and Y. Fainman, "Thermal and Kerr nonlinear properties of plasma-deposited silicon nitride/silicon dioxide waveguides," Optics Express 16(17), 12987-12994 (2008).

[13] R. Halir, Y. Okawachi, J. S. Levy, M. A. Foster, M. Lipson, and A. L. Gaeta, "Ultrabroadband supercontinuum generation in a CMOScompatible platform," Optics Lett. 37(10), 1685-1687 (2012).

[14] J. S. Levy, A. Gondarenko, M. A. Foster, A. C. Turner-Foster, A. L. Gaeta, and M. Lipson, "CMOS-compatible multiple-wavelength oscillator for on-chip optical interconnects," Nature Photon. 4(1), 37-40 (2009).

[15] J. Pfeifle, V. Brasch, M. Lauermann, Y. Yu, D. Wegner, T. Herr, K. Hartinger, P. Schindler, J. Li, D. Hillerkuss, R. Schmogrow, C. Weimann, R. Holzwarth, W. Freude, J. Leuthold, T. J. Kippenberg, and C. Koos, "Coherent terabit communications with microresonator Kerr frequency combs," Nature Photon. 8(5), 375-380 (2014).

[16] G. P. Agrawal, Nonlinear fiber optics, 4th ed., (Academic, 2007).

[17] K. Luke, A. Dutt, C. B. Poitras, and M. Lipson, "Overcoming $\mathrm{Si}_{3} \mathrm{~N}_{4}$ film stress limitations for high quality factor ring resonators," Optics Express 21(19), 22829-22833 (2013).

[18] A. Gondarenko, J. S. Levy, and M. Lipson, "High confinement micron-scale silicon nitride high Q ring resonator," Optics Express 17(14), 11366-11370 (2009).

[19] R. Heideman and M. Hoekman, "Surface waveguide and method of manufacture," US Patent 7,142,759 2 (2006).

[20] TriPleX ${ }^{T M}$ is commercially available through LioniX BV, Enschede, The Netherlands. 


\section{Broadband supercontinuum generation in dispersion engineered stoichiometric silicon nitride waveguides pumped at telecommunication wavelengths}

We report broadband supercontinuum generation in a new type of stoichiometric silicon nitride $\left(\mathrm{Si}_{3} \mathrm{~N}_{4}\right)$ waveguides with increased cross sectional size and rounded geometry. A spectrum more than $700 \mathrm{~nm}$ wide at a -30 dB level is obtained when pumped in the anomalous group velocity dispersion regime at $1560 \mathrm{~nm}$ using an ultrafast erbium-doped fiber laser with 65 fs duration pulses and 43 pJ pulse energy. Based on excellent agreement, detailed numerical calculations show that an octave-spanning supercontinuum with a high degree of coherence over the entire generated bandwidth can be obtained at pulse energies as small as $50 \mathrm{pJ}$. 


\subsection{Introduction}

Supercontinuum generation (SCG) in optical fibers is capable to provide broadband spectra with high spatial coherence [1,2], which enable numerous applications such as in spectroscopy [3], life sciences [4], or metrology [5]. The broad bandwidths in SCG are obtained via nonlinear optical conversion, typically of ultrashort pulses with high peak power that are tightly focused into fibers, while optical guiding maintains high intensities over extended interaction lengths. However, specifically for applications in the telecommunication range it is required to provide SCG with a small footprint, to reduce the required pump laser pulse energies, and to enable integration with pump laser sources.

These requirements can be fulfilled when employing optical waveguides integrated on a chip. Making use of materials that allow for tight optical confinement and that offer an increased nonlinearity, the nonlinear parameter, $\gamma$, can be significantly increased, as compared to silica-based fibers [6]. This enables SCG over short interaction lengths and at relatively low pulse energies $[7,8]$.

Depending on the desired wavelength range of the SCG, the various integrated photonic platforms available offer complementary properties. Materials with a relatively small band gap provide a high nonlinear parameter for SCG, such as chalcogenides $[7,9]$ and silicon $[10,11]$, where a high degree of temporal coherence of the supercontinuum was reported [11]. However, the small band gap limits such platforms to relatively long wavelengths, i.e., the mid-infrared and beyond. At shorter wavelengths, for instance including the $1.5 \mu \mathrm{m}$ telecommunication C-band, SCG in these materials is limited by nonlinear losses due to the combination of high peak intensity required for broadband SCG. Therefore, large band gap silica-based integrated platforms are to be preferred in this spectral range, where nonlinear losses arise at shorter wavelengths. Accordingly, broadband SCG in the near-infrared has been reported in silica [12], doped silica [13], and silicon nitride waveguides [14-16].

Particularly, silicon nitride and doped silica have been reported to be promising materials for integrated nonlinear optics [17] due to their CMOS compatibility and wide optical transparency window. Of these two, silicon nitride offers a significantly higher nonlinearity [18] than doped silica. An additional advantage emerges when using stoichiometric $\mathrm{Si}_{3} \mathrm{~N}_{4}$, deposited by low-pressure chemical vapor deposition (LPCVD). The intrinsic absorption and scattering are both weak [19] and reduce the propagation loss to record values as low as $0.1 \mathrm{~dB} / \mathrm{m}$ [20]. Such excellent performance is to date not possible with $\mathrm{Si}_{\mathrm{x}} \mathrm{N}_{\mathrm{y}}$, deposited by plasma-enhanced chemical vapor deposition (PECVD).

A third advantage of stoichiometric fabrication using LPCVD is a superior reproducibility in the refractive index values, which is a central precondition for dispersion engineering via appropriately designing the waveguide cross section. To obtain anomalous group velocity dispersion (GVD) as needed for efficient SCG, however, rather thick layers of $\mathrm{Si}_{3} \mathrm{~N}_{4}(\sim 600 \mathrm{~nm}$ and more $)$ 
are needed. Fabricating waveguides of the required thickness has proved to be problematic due to the stress induced by the deposition of $\mathrm{Si}_{3} \mathrm{~N}_{4}$, which leads to cracking and severely reduces yield in waveguide fabrication. Recently, we reported a novel fabrication method for high-thickness waveguides (up to $\sim 1 \mu \mathrm{m}$ ) [21]. This new method results in high-yield fabrication of stoichiometric silicon nitride waveguides with a large core area, based on low-loss TriPleX technology [22]. However, the cross-section of the waveguide core shows a nonrectangular shape, which can be expected to have an impact on the waveguide dispersion and thereby also on the shape of the supercontinuum spectra that can be generated.

In this paper, we present an experimental and theoretical investigation of supercontinuum generation in stoichiometric silicon nitride waveguides obtained from our novel fabrication method. We engineer the anomalous GVD of these non-rectangular waveguides to enable SCG pumped in the telecommunication C-band. Based on an excellent agreement of the calculated spectra with the measured spectra, an extrapolation to higher laser pulse energies indicates a strong potential for on-chip, CMOS-compatible SCG with octave-spanning fully coherent spectra.

\subsection{Dispersion calculation}

In order to generate a broadband supercontinuum, the waveguides have to provide anomalous GVD for the pump wavelength. Since SCG depends strongly on the dispersion and to accurately model the obtained supercontinuum, it is important to carefully calculate the dispersion of the waveguides over the entire relevant spectral width. The effective dispersion of the waveguide is given by the dispersion of the two materials used, as well as by the shape and dimensions of the waveguide core. To accurately calculate the effective refractive index of the waveguide modes, we take into account the non-rectangular shape of the waveguide core, which we obtain from scanning electron microscope (SEM) pictures such as in Fig. 5.1(a). It can be seen that, while only the upper part is rectangular, the lower part of the waveguide core resembles a semi-circle. In the calculation of the dispersion, we use a two-dimensional step index profile that resembles the measured core shape and area. We use a finite element solver [23] to calculate the effective refractive index, $n$, and area of the waveguide modes from the index profile for a wavelength range from 500 to $2500 \mathrm{~nm}$. From the wavelength dependent effective refractive index we calculate the GVD of the waveguides and the dispersion parameter, $D=-\frac{\lambda}{c} \cdot \frac{d^{2} n}{d \lambda^{2}}[24]$, for the fundamental quasi-TM mode. Note that the waveguides were designed to exhibit anomalous GVD for the fundamental quasi-TM mode, which is dominantly polarized in the y-direction (see Fig. 5.1(a)). The derived dispersion parameter in waveguides with a height, $h$, of $1.2 \mu \mathrm{m}$ and a width, $w$, ranging from $0.70 \mu \mathrm{m}$ to $0.80 \mu \mathrm{m}$, is shown in Fig. 5.1(b). While bulk $\mathrm{Si}_{3} \mathrm{~N}_{4}$ (red dashed line) exhibits only normal GVD $(D<0)$ in the considered wavelength range, it can be seen 
that the additional dispersion induced by the waveguide geometry results in a wide range of anomalous GVD $(D>0)$ extending over a bandwidth of $440 \mathrm{~nm}$ and $670 \mathrm{~nm}$ for a waveguide width of $0.70 \mu \mathrm{m}$ and $0.80 \mu \mathrm{m}$, respectively.

a)

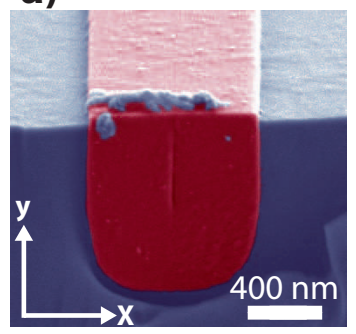

b)

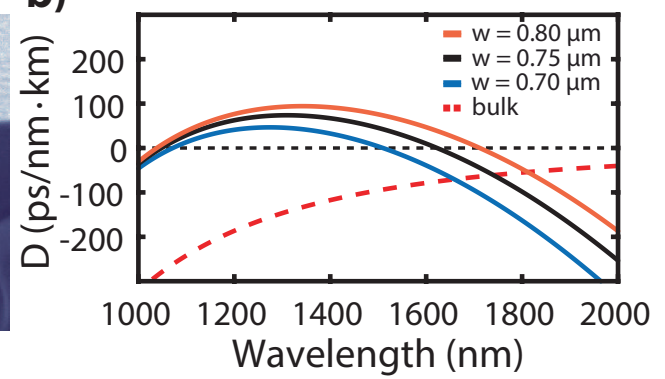

Figure 5.1: (a) SEM picture (false color) of the waveguide facet before deposition of the silica top cladding. (b) Calculated dispersion parameter, $D$, for bulk $S_{3} N_{4}$ (red dashed line) and for the fundamental quasi-TM mode in $\mathrm{Si}_{3} \mathrm{~N}_{4}$ waveguides with a height $h=1.2 \mu \mathrm{m}$ and various waveguide widths of $w=0.70 \mu \mathrm{m}$ (blue), $0.75 \mu \mathrm{m}$ (black), and $0.80 \mu \mathrm{m}$ (orange). The calculations take the rounded shape of the waveguide core into account [21]. The predicted dispersion can be designed to set the longer zero dispersion wavlength (ZDW) to $1510 \mathrm{~nm}$ using $w=0.70 \mu \mathrm{m}$ or to $1712 \mathrm{~nm}$ when using $w=0.80 \mu \mathrm{m}$, such that the range with $D>0$ includes the pump wavelength of $1560 \mathrm{~nm}$.

\subsection{Experimental results}

Unlike previously reported experiments on SCG in stoichiometric $\mathrm{Si}_{3} \mathrm{~N}_{4}$ waveguides $[14,16]$, in which bulk, high-power pump sources such as a titaniumsapphire laser or an optical parametric oscillator were employed, here we use a mode-locked, erbium-doped fiber laser. Well developed erbium-doped fiber lasers are widely available due to their importance for optical telecommunications, and they are already commonly used to generate optical frequency combs via SCG in optical fibers [25]. A highly interesting option for future, fully integrated SCG on a chip is that erbium-doped lasers can be integrated within the $\mathrm{Si}_{3} \mathrm{~N}_{4}$ platform [26] where they provide rather high average power of $75 \mathrm{~mW}$. The fiber laser used in this experiment is based on the design reported by $\mathrm{Ma}$ et al. [27] and its center wavelength lies at $1560 \mathrm{~nm}$. The laser has a repetition rate of $220 \mathrm{MHz}$, a full width half maximum (FWHM) spectral width of $72 \mathrm{~nm}$, and generates pulses that are compressed to a FWHM pulse duration of $65 \mathrm{fs}$ (assuming Gaussian pulses) using a pair of prisms. The timebandwidth-product of 0.58 after compression indicates that the pulses are close to the transform limit of Gaussian pulses. The pulses are coupled into a $5.5 \mathrm{~mm}$ long $\mathrm{Si}_{3} \mathrm{~N}_{4}$ waveguide using an aspheric lens (incoupling loss of $8 \mathrm{~dB}$ ), while the 
input polarization is set by a half-wave plate to excite quasi-TM modes. The supercontinuum is collected by using a lensed fiber and is characterized with an optical spectrum analyzer (Ando AQ 6317). To minimize the collection of undesired scattered light during the measurements, the waveguide bends twice to achieve a lateral offset of $0.6 \mathrm{~mm}$ between the input and output waveguide facets of the chip. To ensure that the pump wavelength experiences anomalous GVD, for allowing a broad supercontinuum spectrum, a waveguide cross section of $h=1.2 \mu \mathrm{m}$ and $w=0.75 \mu \mathrm{m}$ is chosen.
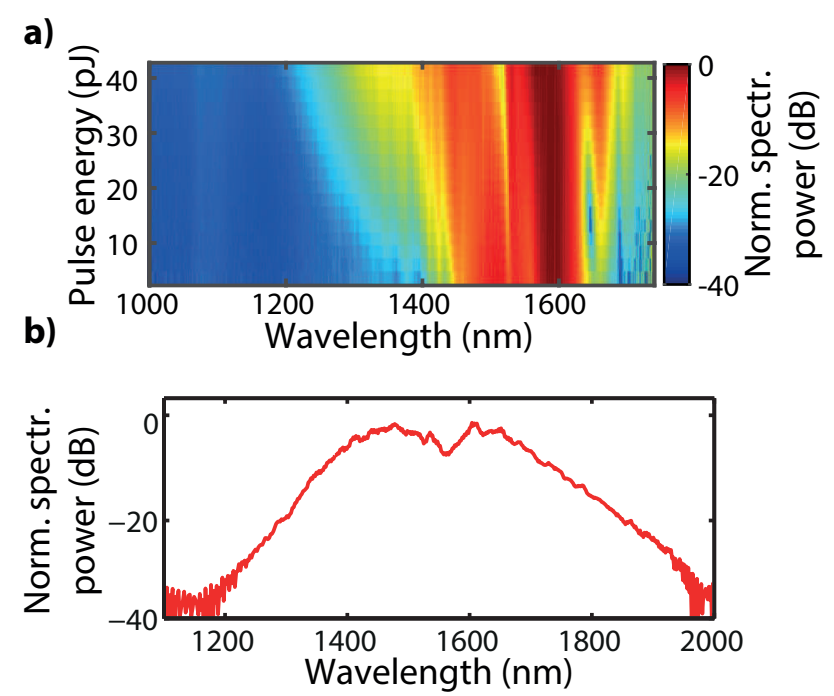

Figure 5.2: (a) Measured spectral evolution of the supercontinuum with a spectral resolution of $2 \mathrm{~nm}$ as a function of the incoupled pulse energy after propagating through a $5.5 \mathrm{~mm}$ long $\mathrm{Si}_{3} \mathrm{~N}_{4}$ waveguide. The spectrum broadens monotonically with increasing pulse energy. The recorded spectra are limited to $1750 \mathrm{~nm}$ due to the spectral range of the optical spectrum analyzer. To visualize the broadening, each spectrum was normalized to the peak of the spectrum. (b) Normalized output spectrum at the maximum available pulse energy of $43 \mathrm{pJ}$ recorded with a spectrometer of wider range. The spectrum extends from $1225 \mathrm{~nm}$ to $1940 \mathrm{~nm}$, covering more than $700 \mathrm{~nm}$ at a $-30 \mathrm{~dB}$ level.

Figure 5.2(a) displays a series of measured supercontinuum spectra and shows how their bandwidth depends on the incoupled pulse energy, $E_{p}$, of the pump laser. It can be seen that the width of the spectrum continuously broadens with increasing $E_{p}$ up to our maximum available pulse energy ( $E_{p}=43 \mathrm{pJ}$ ). For extending the observable spectral range beyond the instrumental limit of the OSA at $1750 \mathrm{~nm}$, the complete spectrum was measured using a second spectrometer (NIRLine, Avantes BV) with a spectral range from $1000 \mathrm{~nm}$ to $2000 \mathrm{~nm}$ and a spectral resolution of $10 \mathrm{~nm}$ (see Fig. 5.2(b) recorded at $E_{p}=43 \mathrm{pJ}$ ). The shown spectrum extends from $1230 \mathrm{~nm}$ to $1960 \mathrm{~nm}$ (at a 
$-30 \mathrm{~dB}$ level) covering a spectral range of more than $700 \mathrm{~nm}(\approx 90 \mathrm{THz})$. An important feature of the recorded supercontinuum spectra as shown in Fig. 5.2 is that the overall spectral shape is rather smooth, resembling a wide Gaussian, not exhibiting additional features such as dispersive waves. From this it can be concluded that the width of the obtained supercontinuum spectra is currently limited only by the available pump energy and not by the bandwidth of the anomalous GVD regime.

\subsection{Theoretical modeling}

For an understanding of the shape of the measured supercontinuum spectra and their relation to the waveguide dispersion, and for exploring the potential of the used waveguides, we performed numerical calculations of the nonlinear pulse propagation using the generalized nonlinear Schrödinger (GNLSE) equation [6, 28]. The calculation parameters include the nonlinear parameter $\gamma=1.21 \mathrm{~m}^{-1} \mathrm{~W}^{-1}$ and up to the 9 th order of dispersion. For taking into account the wavelength-dependent increase of $\gamma$ a shock term, $\tau_{s}$, is introduced and calculated to be $1.6 \mathrm{fs}$ [6], which also takes the decrease of effective mode area of the fundamental mode at shorter wavelengths into account. We approximate the contribution of the Raman effect in our calculations by data known from silica fibers [29], because sufficient experimental data on the Raman gain in $\mathrm{Si}_{3} \mathrm{~N}_{4}$ waveguides is not available. A propagation loss, $\alpha$, of $1.35 \mathrm{~dB} / \mathrm{cm}$ is taken into account as we found in previous measurements [21]. Finally, to improve the accuracy of the calculated supercontinuum spectrum, we use the measured spectrum of the fiber laser as input for the numerical calculations.

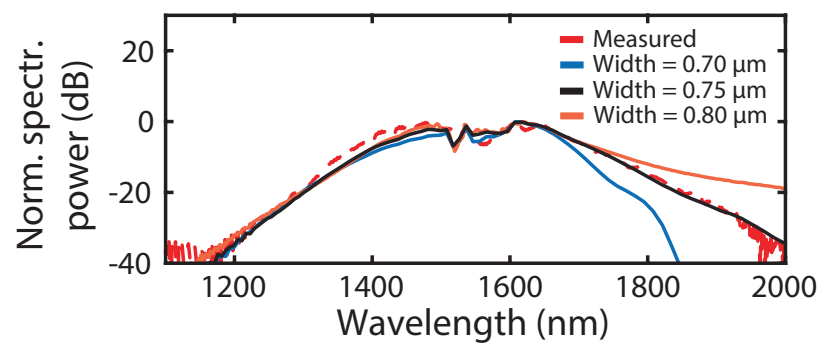

Figure 5.3: Measured (red dashed line) and calculated (black) supercontinua generated in $\mathrm{Si}_{3} \mathrm{~N}_{4}$ waveguides with a height $h=1.2 \mu \mathrm{m}$ and $w=0.75 \mu \mathrm{m}$. Calculated supercontina are also shown for $w=0.70 \mu m$ (blue) and $w=0.80 \mu m$ (orange). The input pulse energies for the calculated spectra are chosen to be be $48 \mathrm{pJ}, 36 \mathrm{pJ}$, and $33 \mathrm{pJ}$ for $w=0.70 \mu \mathrm{m}, 0.75 \mu \mathrm{m}$, and $0.80 \mu \mathrm{m}$, respectively, to match the measured spectrum of a pump energy of $43 \mathrm{pJ}$ (red dashed line).

The measured supercontinuum spectrum for a waveguide with $w=0.75 \mu \mathrm{m}$ 
and an incoupled pump energy of $43 \mathrm{pJ}$ is shown in Fig. 5.3 together with calculated spectra for waveguide widths $w$ from $0.70 \mu \mathrm{m}$ to $0.80 \mu \mathrm{m}$. The pump energy, $E_{p}$, was used as a fit parameter and adjusted to provide the best match with the measured spectrum, resulting in an assumed $E_{p}$ of $48 \mathrm{pJ}$ $(w=0.70 \mu \mathrm{m}), 36 \mathrm{pJ}(w=0.75 \mu \mathrm{m})$, and $33 \mathrm{pJ}(w=0.8 \mu \mathrm{m})$, respectively. It can be seen that the calculated spectrum for $w=0.75 \mu \mathrm{m}$ (black line) is in excellent agreement with the measured spectrum (red dashed line), which indicates that the calculated dispersion parameters are accurate and that the theoretical model describes the observed supercontinuum correctly to large detail. We address the small difference between the measured and modeled pulse energy to some deviation in the temporal profile of the pulse emitted by the laser as compared to an assumed Gaussian pulse shape.

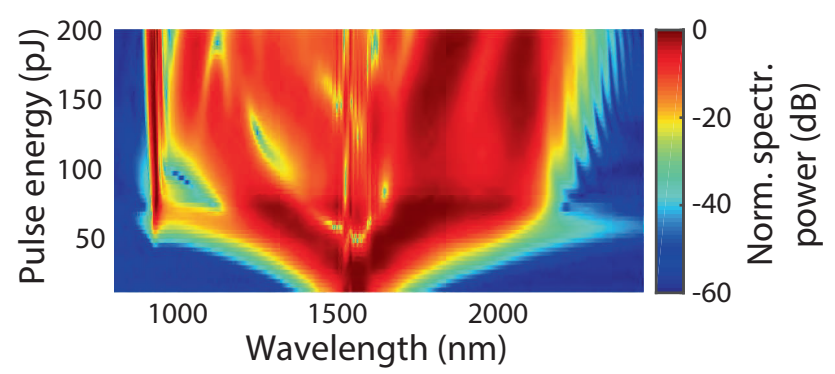

Figure 5.4: Numerical calculation of the spectral broadening with increasing incoupled pulse energy of up to $200 \mathrm{pJ}$ for a waveguide with $h=1.0 \mu \mathrm{m}$ and $w=0.75 \mu \mathrm{m}$.

For potential use in self-referencing frequency combs, it is important that the spectra span across one octave and more. As the supercontinuum spectrum broadens with increasing input pulse energy as shown in Fig. 5.2(a), we numerically investigate if octave-spanning SCG can be realized with these waveguides if higher input energies are available. The calculated spectrum as a function of the input pulse energy is shown in Fig. 5.4 for a waveguide with $h=1.2 \mu \mathrm{m}$ and $w=0.75 \mu \mathrm{m}$, which shows the best match with experimental data. From Fig. 5.4 it can be seen that the supercontinuum spectrum easily becomes octave-spanning (at a $-30 \mathrm{~dB}$ level) for pulse energies as low as $50 \mathrm{pJ}$. These results confirm that no dispersive waves are present for the range of input pulse energies available in the experiment and that the supercontinuum spectra are relative smooth in agreement with the measurements as shown in Fig. 5.2(a). Only beyond an input pulse energy of more than $50 \mathrm{pJ}$ does the supercontinuum spectrum show the presence of two dispersive waves, which are centered around $930 \mathrm{~nm}$ and $2060 \mathrm{~nm}$ at the short and long wavelength part of the spectrum, respectively. The total expected bandwidth of the supercontinuum is $1320 \mathrm{~nm}$ at a $-30 \mathrm{~dB}$ level with an $E_{p}$ of $100 \mathrm{pJ}$.

An additional important parameter in optical frequency combs is the pulseto-pulse coherence of the supercontinuum spectra. Our model is well suited to calculate the pulse-to-pulse coherence as it contains inherently coherent pro- 


\section{a)}
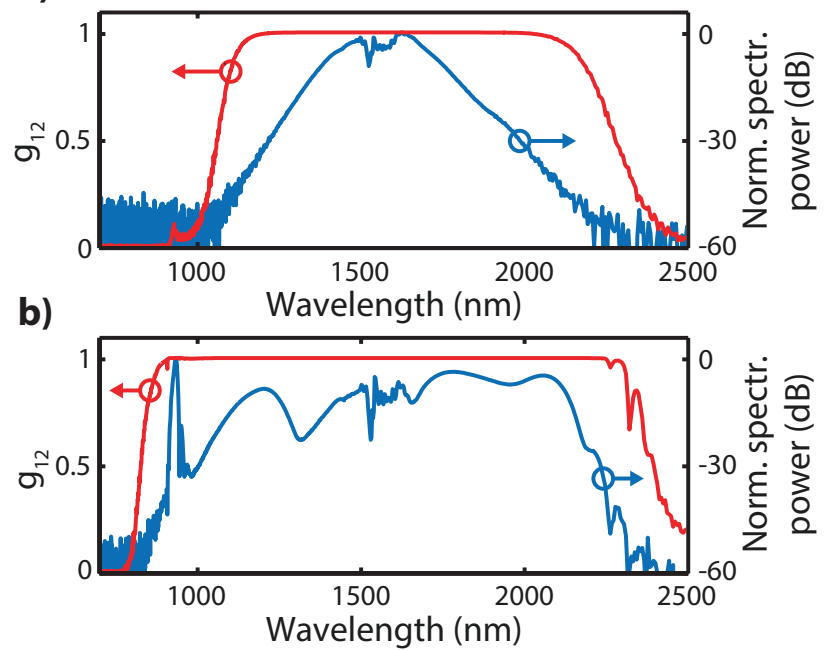

Figure 5.5: Calculated coherence as a function of wavelength in red and one corresponding spectrum out of the ensemble in blue for two pulse energies of (a) $36 \mathrm{pJ}$ and (b) $100 \mathrm{pJ}$ in a waveguide with $h=1.2 \mu \mathrm{m}, w=0.75 \mu \mathrm{m}$, and $5.5 \mathrm{~mm}$ length.

cesses, e.g., self and cross-phase modulation, as well as fundamentally incoherent processes, e.g., spontaneous Raman scattering and four-wave mixing starting up spontaneously from vacuum fluctuations. To show that the broadened spectra generated by our model are dominated by coherent processes and possess a high degree of coherence, we determine the cross coherence (mutual coherence) of different pulses in a train, based on an ensemble of calculated spectra following the procedure reported by Ruehl et al. [2]. For an ensemble of 1000 supercontinuum spectra, in which a stochastic distribution of shot noise [30] was added to each input pulse to include the influence of vacuum fluctuations, we calculate the first-order coherence function:

$$
g_{12}(\omega)=\frac{\left|\left\langle E_{i}(\omega) E_{j}^{*}(\omega)\right\rangle_{i \neq j}\right|}{\sqrt{\left\langle\left|E_{i}(\omega)\right|^{2}\right\rangle\left\langle\left|E_{j}(\omega)\right|^{2}\right\rangle}} .
$$

In Fig. 5.5 the calculated coherence is shown as a function of wavelength (red traces) for two input pulse energies, (a) for $36 \mathrm{pJ}$, which shows the spectral best match with measured data, and (b) for $100 \mathrm{pJ}$ (extrapolation to stronger pumping). For comparison, the corresponding supercontinuum spectra are shown in blue. It can be seen that for $E p=36 \mathrm{pJ}$, where the calculated supercontinuum power spectrum shows excellent agreement with the measured spectrum, the coherence function, $g_{12}$, equals 1 from $1200 \mathrm{~nm}$ to $2050 \mathrm{~nm}$, i.e., the supercontinuum spectrum is fully coherent. Note that this range of full coherence 
coincides with the range where the power of the spectrum is larger than $-30 \mathrm{~dB}$. The same is true for the results obtained for $E_{p}=100 \mathrm{pJ}$, where is $g_{12}=1$ over the spectral range with a power $>-30 \mathrm{~dB}$ as shown in Fig. 5.5(b), despite the presence of features in the spectrum. The observation of full coherence for parameters as used in the experiments indicates that the spectral broadening is to a dominant extent a result of coherent nonlinear optical processes.

\subsection{Conclusion}

In conclusion, we have shown broadband supercontinuum generation in a novel type of $\mathrm{Si}_{3} \mathrm{~N}_{4}$ waveguides with a bandwidth of more than $700 \mathrm{~nm}$. The supercontinuum spectrum spans over the complete telecommunication wavelength range when pumped with a mode-locked sub-100 fs erbium-doped fiber laser. The obtained supercontinuum spectrum is in excellent agreement with our model based on the nonlinear Schrödinger equation when taking the nonrectangular shape of our waveguides into account. Extrapolation to slightly higher pump energies of $50 \mathrm{pJ}$ predicts that the bandwidth of the supercontinuum is further increased and becomes octave-spanning. The required increase in pump energy should be easily attainable via integrating inversely tapered mode converters as was reported for efficient fiber-to-chip coupling with less than $0.5 \mathrm{~dB}$ loss for the TriPleX platform [22]. Justified by the excellent agreement of the theoretical power spectra, calculations show low pulse-to-pulse variations and reveal a high coherence of the supercontinuum. This would enable self-referencing frequency combs based on $f-2 f$ and $2 f-3 f$ interferometry operating at low average output power in the telecommunication wavelength range.

\section{Acknowledgments}

This research is supported by the Dutch Technology Foundation STW, which is part of the Netherlands Organisation for Scientific Research (NWO), and which is partly funded by the Ministry of Economic Affairs. 



\section{Bibliography}

[1] D. J. Jones, S. A. Diddams, J. K. Ranka, A. Stentz, R. S. Windeler, J. L. Hall, and S. T. Cundiff, "Carrier-envelope phase control of femtosecond mode-locked lasers and direct optical frequency synthesis," Science 288, 635 (2000).

[2] A. Ruehl, M. J. Martin, K. C. Cossel, L. Chen, H. McKay, B. Thomas, C. Benko, L. Dong, J. M. Dudley, M. E. Fermann, I. Hartl, and J. Ye, "Ultrabroadband coherent supercontinuum frequency comb," Phys. Rev. A 84, 3 (2011).

[3] H. Kano and H.-O. Hamaguchi, "Characterization of a supercontinuum generated from a photonic crystal fiber and its application to coherent Raman spectroscopy," Optics Lett. 28, 2360 (2003).

[4] G. Humbert, W. Wadsworth, S. Leon-Saval, J. Knight, T. Birks, P. St. J. Russell, M. Lederer, D. Kopf, K. Wiesauer, E. Breuer, and D. Stifter, "Supercontinuum generation system for optical coherence tomography based on tapered photonic crystal fibre," Optics Express 14, 1596 (2006).

[5] J. T. Woodward, A. W. Smith, C. A. Jenkins, C. Lin, S. W. Brown, and K. R. Lykke, "Supercontinuum sources for metrology," Metrologia 46, S277 (2009).

[6] J. M. Dudley and S. Coen, "Supercontinuum generation in photonic crystal fiber," Rev. Mod. Phys. 78, 1135 (2006).

[7] M. R. Lamont, B. Luther-Davies, D.-Y. Choi, S. Madden, and B. J. Eggleton, "Supercontinuum generation in dispersion engineered highly nonlinear $(\gamma=10 / \mathrm{W} / \mathrm{m}) \mathrm{As}_{2} \mathrm{~S}_{3}$ chalcogenide planar waveguide," Optics Express 16, 14938 (2008).

[8] I. W. Hsieh, X. Chen, X. Liu, J. I. Dadap, N. C. Panoiu, C. Y. Chou, F. Xia, W. M. Green, Y. A. Vlasov, and R. M. Osgood, "Supercontinuum generation in silicon photonic wires," Optics Express 15, 15242 (2007).

[9] Y. Yu, X. Gai, P. Ma, D. Y. Choi, Z. Yang, R. Wang, S. Debbarma, S. J. Madden, and B. Luther-Davies, "A broadband, quasi-continuous, midinfrared supercontinuum generated in a chalcogenide glass waveguide," Laser Photon. Rev. 798, 792 (2014). 
[10] R. K. W. Lau, M. R. E. Lamont, A. G. Griffith, Y. Okawachi, M. Lipson, and A. L. Gaeta, "Octave-spanning mid-infrared supercontinuum generation in silicon nanowaveguides," Optics Lett. 39, 4518 (2014).

[11] B. Kuyken, T. Ideguchi, S. Holzner, M. Yan, T. W. Hänsch, J. Van Campenhout, P. Verheyen, S. Coen, F. Leo, R. Baets, G. Roelkens, and N. Picqué, "An octave-spanning mid-infrared frequency comb generated in a silicon nanophotonic wire waveguide," Nature Commun. 6, 6310 (2015).

[12] D. Y. Oh, D. Sell, H. Lee, K. Y. Yang, S. A. Diddams, and K. J. Vahala, "Supercontinuum generation in an on-chip silica waveguide," Optics Lett. 39, 1046 (2014).

[13] D. Duchesne, M. Peccianti, M. R. E. Lamont, M. Ferrera, L. Razzari, F. Légaré, R. Morandotti, S. Chu, B. E. Little, and D. J. Moss, "Supercontinuum generation in a high index doped silica glass spiral waveguide," Optics Express 18, 923 (2010).

[14] R. Halir, Y. Okawachi, J. S. Levy, M. A. Foster, M. Lipson, and A. L. Gaeta, "Ultrabroadband supercontinuum generation in a CMOScompatible platform," Optics Lett. 37, 1685 (2012).

[15] J. M. Chavez Boggio, D. Bodenmüller, T. Fremberg, R. Haynes, M. M. Roth, R. Eisermann, M. Lisker, L. Zimmermann, and M. Böhm, "Dispersion engineered silicon nitride waveguides by geometrical and refractiveindex optimization," J. Opt. Soc. Am. B 31, 2846 (2014).

[16] H. Zhao, B. Kuyken, S. Clemmen, F. Leo, A. Subramanian, A. Dhakal, P. Helin, S. Severi, E. Brainis, G. Roelkens, and R. Baets, "Visible-to-nearinfrared octave spanning supercontinuum generation in a silicon nitride waveguide," Optics Lett. 40, 2177 (2015).

[17] D. J. Moss, R. Morandotti, A. L. Gaeta, and M. Lipson, "New CMOScompatible platforms based on silicon nitride and Hydex for nonlinear optics," Nature Photon. 7, 597 (2013).

[18] K. Ikeda, R. E. Saperstein, N. Alic, and Y. Fainman, "Thermal and Kerr nonlinear properties of plasma-deposited silicon nitride/silicon dioxide waveguides," Optics Express 16, 12987 (2008).

[19] A. Gondarenko, J. S. Levy, and M. Lipson, "High confinement micron-scale silicon nitride high Q ring resonator," Optics Express 17, 11366 (2009).

[20] J. F. Bauters, M. J. R. Heck, D. John, D. Dai, M.-C. Tien, J. S. Barton, A. Leinse, R. G. Heideman, D. J. Blumenthal, and J. E. Bowers, "Ultra-low-loss high-aspect-ratio $\mathrm{Si}_{3} \mathrm{~N}_{4}$ waveguides," Optics Express 19, 3163 (2011). 
[21] J. P. Epping, M. Hoekman, R. Mateman, A. Leinse, R. G. Heideman, A. van Rees, P. J. van der Slot, C. J. Lee, and K.-J. Boller, "High confinement, high yield $\mathrm{Si}_{3} \mathrm{~N}_{4}$ waveguides for nonlinear optical applications," Optics Express 23, 642 (2015).

[22] K. Wörhoff, R. G. Heideman, A. Leinse, and M. Hoekman, "TriPleX: a versatile dielectric photonic platform," Adv. Opt. Techn. 4, 189 (2015).

[23] A. B. Fallahkhair, K. S. Li, and T. E. Murphy, "Vector finite difference modesolver for anisotropic dielectric waveguides," J. Lightwave Technol. 26, 1423 (2008).

[24] G. Agrawal, Nonlinear Fiber Optics, 4th ed., (Academic, 2007).

[25] G. Ycas, S. Osterman, and S. A. Diddams, "Generation of a 660-2100 nm laser frequency comb based on an erbium fiber laser," Optics Lett. 37, 2199 (2012).

[26] E. S. Hosseini, P. Purnawirman, J. D. B. Bradley, J. Sun, G. Leake, T. N. Adam, D. D. Coolbaugh, and M. R. Watts, "CMOS-compatible $75 \mathrm{~mW}$ erbium-doped distributed feedback laser," Optics Lett. 39, 3106 (2014).

[27] D. Ma, Y. Cai, C. Zhou, W. Zong, L. Chen, and Z. Zhang, "37.4 fs pulse generation in an Er:fiber laser at a $225 \mathrm{MHz}$ repetition rate," Optics Lett. 35, 2858 (2010).

[28] M. Kues, N. Brauckmann, T. Walbaum, P. Gross, and C. Fallnich, "Nonlinear dynamics of femtosecond supercontinuum generation with feedback," Optics Express 17, 15827 (2009).

[29] R. H. Stolen, J. P. Gordon, W. J. Tomlinson, and H. A. Haus, "Raman response function of silica-core fibers," J. Opt. Soc. Am. B 6(6), 1159 (1989).

[30] R. Paschotta, "Noise of mode-locked lasers (Part I): numerical model," Appl. Phys. B 79, 153 (2004). 



\section{On-chip visible-to-infrared supercontinuum generation with more than $495 \mathrm{THz}$ spectral bandwidth}

We report on ultra-broadband supercontinuum generation in highconfinement $\mathrm{Si}_{3} \mathrm{~N}_{4}$ integrated optical waveguides. The spectrum extends through the visible (from $470 \mathrm{~nm}$ ) to the infrared spectral range $(2130 \mathrm{~nm})$ comprising a spectral bandwidth wider than $495 \mathrm{THz}$, which is the widest supercontinuum spectrum generated on a chip. ${ }^{1}$

\footnotetext{
${ }^{1}$ This Chapter has been published as "On-chip visible-to-infrared supercontinuum generation with more than 495 THz spectral bandwidth," Optics Express 23(15), 19596 (2015).
} 


\subsection{Introduction}

Ultra-broadband optical spectra obtained by supercontinuum generation (SCG) have found wide-spread use in applications and fundamental research. Examples are optical coherence tomography [1], unraveling nonlinear dynamics [2-4], coherent spectroscopy [5], and frequency metrology [6]. Of particular interest is extending SCG into the visible range and beyond, which has so far been possible only with bulk nonlinear media [7], tapered fibers [8, 9], photonic crystal fibers [10-12], and $\chi^{(2)}$ nonlinear waveguides [13].

A major disadvantage of these approaches is their lack of CMOS compatibility, because CMOS compatibility allows for fabrication of devices in high volumes using established facilities and for photonic integration with electronic circuits [14]. A well-known CMOS-compatible waveguide platform is provided in silicon photonics, where a large nonlinear coefficient $\left(\gamma \approx 300 \mathrm{~W}^{-1} \mathrm{~m}^{-1}\right)$ is available to overcome waveguide losses and provide highly efficient SCG $[15,16]$. However, the small band gap of silicon, corresponding to about $1 \mu \mathrm{m}$ wavelength, limits the wavelength range of supercontinua to the infrared. Another limit is that at the high intensities needed for ultra-broadband SCG, pump wavelengths shorter than $2 \mu \mathrm{m}$, such as in the 1.3 and $1.5 \mu \mathrm{m}$ telecommunication bands, suffer from nonlinear losses occurring in silicon such as two-photon absorption and subsequent free-carrier absorption.

A most promising CMOS-compatible approach for extending on-chip SCG into the visible range is based on silica $[17,18]$ and silicon nitride [19-21] glass platforms, which offer excellent transparency down to the ultraviolet. Specifically, low-pressure chemical vapor deposition (LPCVD) of stoichiometric materials [22] offers highly reproducible fabrication with predefined material dispersion and extremely low-loss waveguides $(<0.1 \mathrm{~dB} / \mathrm{m})$ [23]. For optimally exploiting the Kerr index of $\mathrm{Si}_{3} \mathrm{~N}_{4}, n_{2}=2.4 \cdot 10^{-19} \mathrm{~m}^{2} / \mathrm{W}$ [24], the optical mode can be tightly confined due to the high-contrast offered by the silicon nitride based glass platform, thereby offering an increased nonlinear coefficient $(\gamma \approx$ $\left.1 \mathrm{~W}^{-1} \mathrm{~m}^{-1}\right)$. Additional advantages are the options available for integrating extended waveguide circuitries [25], as well as rare-earth-doped lasers [26].

The widest supercontinuum spectra obtained in CMOS-compatible platforms to date were generated with silicon nitride waveguides, using ultrashort pulses at $1.5 \mu \mathrm{m}$ wavelength in a $1.4 \mathrm{~cm}$ long waveguide [20] and at $1.3 \mu \mathrm{m}$ wavelength in a $4.3 \mathrm{~cm}$ long waveguide [19]. The latter yielded some output at the edge of the visible range $(>665 \mathrm{~nm})$. Improved engineering of the dispersion was proposed [27] and applied for further spectral broadening [20, 28], however, for SCG, the output remained at wavelengths longer than $700 \mathrm{~nm}$. Most recent experiments successfully extended the SCG towards the blue range [21] with a spectral bandwidth of $310 \mathrm{THz}$ in a $1 \mathrm{~cm}$ long underetched waveguide. These results show that, to obtain shorter wavelength supercontinua on a chip, a shorter wavelength pump is required and, consequently, suitable dispersion for a shorter pump wavelength has to be provided. 
Here, we report ultra-broadband supercontinuum generation in highconfinement stoichiometric $\mathrm{Si}_{3} \mathrm{~N}_{4}$ integrated optical waveguides. The SCG extends from the blue wavelength range $(470 \mathrm{~nm})$ to the infrared spectral range $(2130 \mathrm{~nm})$. This range comprises a spectral bandwidth of more than $495 \mathrm{THz}$ which is, to our knowledge, the widest supercontinuum ever generated on a chip. The generation of such a wide spectral bandwidth supercontinuum is made possible by our novel technique for reliable fabrication of high thickness (up to $\sim 1 \mu \mathrm{m}$ ), crack-free waveguides using $\operatorname{LPCVD}[29,30]$. The increased waveguide dimensions are used to shift the zero dispersion wavelength (ZDW) to values as short as $1 \mu \mathrm{m}$ for phase-matched excitation with a mode-locked $1064 \mathrm{~nm}$ Yb-fiber laser, thereby extending the short-wavelength edge of the supercontinuum and obtain ultra-broadband SCG at a propagation length as short as $5.5 \mathrm{~mm}$.

\subsection{Dispersion calculation}

The scanning electron microscope (SEM) picture in Fig. 6.1(a) shows a typical example of the cross section of the waveguides used in this experiment with a $\mathrm{Si}_{3} \mathrm{~N}_{4}$ waveguide core shown in red and a $\mathrm{SiO}_{2}$ cladding shown in blue. It can be seen that the lower part of the cross section has a rounded shape. For calculating the waveguide dispersion, this shape was taken into account in the form of step index profiles as found in the SEM images. The optical modes and their effective refractive indices were calculated using a finite element solver [31] for a wavelength range from 400 to $2500 \mathrm{~nm}$. The calculated dispersion parameter, $D$, for the fundamental quasi-TM modes and waveguides with a height, $h$, of $1.0 \mu \mathrm{m}$ and widths, $w$, of $0.70,0.75$, and $0.80 \mu \mathrm{m}$, respectively, is shown in Fig. 6.1(b). These particular dimensions were chosen so that light with a pump wavelength of $1064 \mathrm{~nm}$, such as conveniently available from Yb or Nd-doped lasers, experiences anomalous dispersion $(D>0)$, which is not the case for bulk $\mathrm{Si}_{3} \mathrm{~N}_{4}$ (red dashed line). We note that increasing the waveguide width (from $0.70 \mu \mathrm{m}$ to $0.80 \mu \mathrm{m}$ ) increases the wavelength range that experiences anomalous dispersion by $170 \mathrm{~nm}$. This means that a broader bandwidth is expected from wider waveguides. Another result of the increased waveguide dimensions is that the waveguide supports three modes at the pump wavelength in the TM polarization, namely the fundamental $\mathrm{TM}_{00}$ and two higher order modes $\mathrm{TM}_{10}$ and $\mathrm{TM}_{20}$.

\subsection{Experimental results}

The experimental setup for SCG is depicted in Fig. 6.2. Ultrashort pulses with a center wavelength of $1064 \mathrm{~nm}$ are provided with a mode-locked Yb-doped fiber laser (Ekspla, LightWire FF200). The laser provides pulses with about 115 fs duration, a repetition rate of $41 \mathrm{MHz}$, and a maximum average output 

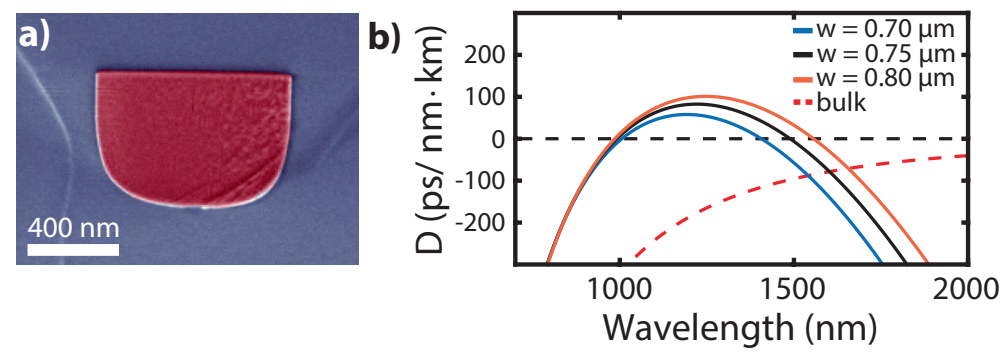

Figure 6.1: (a) SEM picture (false color) of a waveguide facet. (b) Calculated dispersion parameter, D, for bulk $S i_{3} N_{4}$ (red dashed line) and $\mathrm{Si}_{3} \mathrm{~N}_{4}$ waveguides with $h=1.0 \mu \mathrm{m}$ and various waveguide widths, w, of $0.70 \mu \mathrm{m}$ (blue), $0.75 \mu \mathrm{m}$ (black), and $0.80 \mu \mathrm{m}$ (orange) for fundamental quasi-TM modes. The calculations take into account the rounded shape of the waveguides [29]. As can be seen, the range of anomalous dispersion $(D>0)$ increases with the waveguide width, allowing the two zero dispersion wavelengths $(Z D W)$ to be tuned from $1010 \mathrm{~nm}$ and $1410 \mathrm{~nm}$ at $w=0.70 \mu \mathrm{m}$ to $990 \mathrm{~nm}$ and $1560 \mathrm{~nm}$ at $w=0.80 \mu \mathrm{m}$.

power of $200 \mathrm{~mW}$ (maximum pulse energy $5 \mathrm{~nJ}$ ). The pulses are coupled into the $\mathrm{Si}_{3} \mathrm{~N}_{4}$ waveguides with a coupling loss of about $9 \mathrm{~dB}$ using an aspheric lens. The two particular chips selected here, out of 40 chips, contain 20 waveguides of various widths each with a height of $1.0 \mu \mathrm{m}$, and a length of $5.5 \mathrm{~mm}$. To control the pump power, a half-wave plate and a polarizing beam splitter are used. A second half-wave plate is used to set the input polarization for excitation of quasi-TM modes. The supercontinuum is collected using a lensed fiber and recorded using an optical spectrum analyzer (Ando AQ6315A) and a near-infrared spectrometer (Avantes NIRline). To minimize the collection of undesired scattered light during the measurements, the waveguides have two bends that provide an offset, $d$ in Fig. 6.2 , of $0.6 \mathrm{~mm}$ between the input and output waveguide facets.

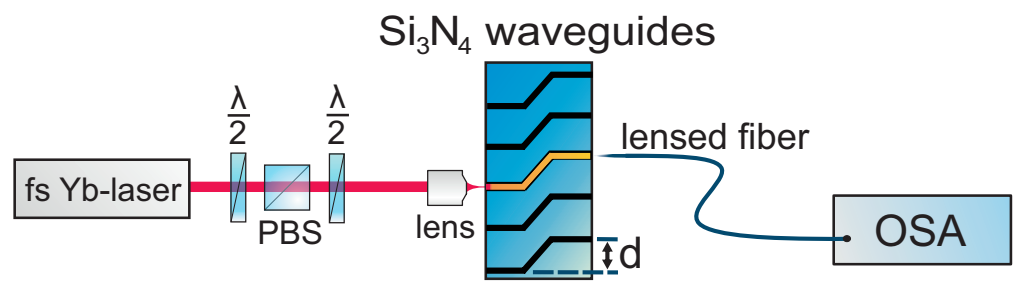

Figure 6.2: Experimental setup for on-chip supercontinuum generation. $\lambda / 2:$ half-wave plate; PBS: polarizing beam splitter; lens: aspheric lens; OSA: optical spectrum analyzer, $d$ : offset between facets to reduce the collection of scattered light. 
The supercontinuum spectrum generated in a waveguide with $w=0.8 \mu \mathrm{m}$, at the maximum available incoupled pulse energy, $E_{p}$, of $590 \mathrm{pJ}$ is shown in Fig. 6.3(a). This pulse energy is limited by the coupling losses. The generated spectrum extends from the blue, at $470 \mathrm{~nm}$ at a $-30 \mathrm{~dB}$ level, and ends in the infrared at $2130 \mathrm{~nm}$. This is, to our knowledge, the widest supercontinuum ever generated on a chip, measured in relative bandwidth (more than 2.1 octaves) as well as absolute bandwidth (more than $495 \mathrm{THz}$ ).
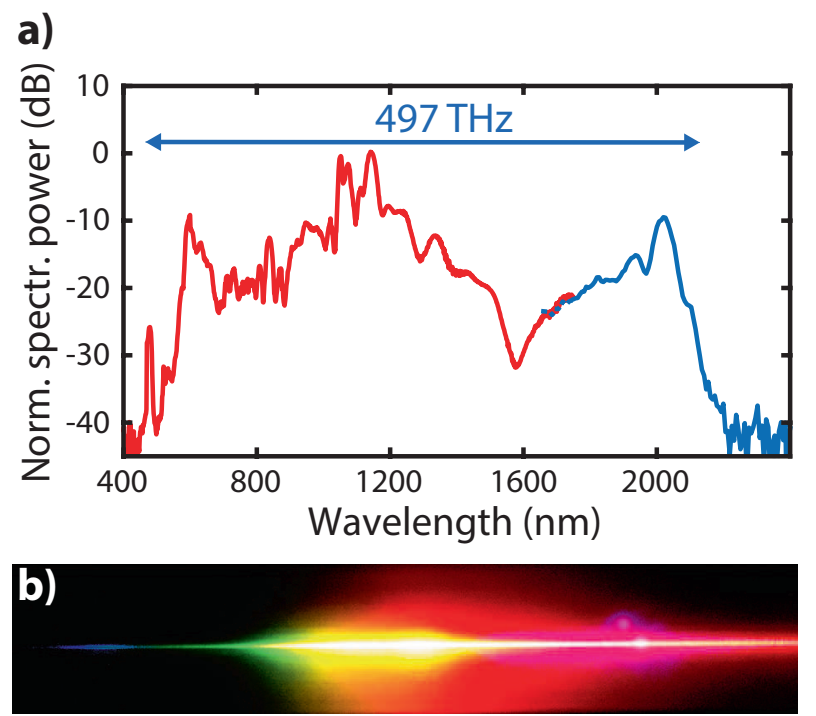

Figure 6.3: (a) Supercontinuum spectrum generated in a $5.5 \mathrm{~mm}$ long $\mathrm{Si}_{3} N_{4}$ waveguide with $h=1.0 \mu \mathrm{m}, w=0.8 \mu \mathrm{m}$, and $E_{p}=590 \mathrm{pJ}$. The spectrum extends from $470 \mathrm{~nm}$ (at a $-30 \mathrm{~dB}$ level) to $2130 \mathrm{~nm}$, which is more than $495 \mathrm{THz}$. The spectrum is measured using an OSA (red) and a near-infrared spectrometer (blue). (b) Photograph of the spectrum after being dispersed by a diffraction grating.

To more systematically characterize the supercontinuum, we measured the output spectra versus incoupled pulse energy of the pump laser. The results for a waveguide with $w=0.775 \mu \mathrm{m}$ are summarized in Fig. 6.4. One can see that the spectrum continuously broadens with increasing $E_{p}$. A dispersive wave becomes noticeable at $E_{p}=355 \mathrm{pJ}$ with its peak at $648 \mathrm{~nm}$. The peak shifts to shorter wavelengths as the power increases, until it reaches $635 \mathrm{~nm}$ at $E_{p}=462 \mathrm{pJ}$. In these experiments, no damaging of the waveguides occurred. However, we found a damage threshold of $1 \mathrm{~nJ}$ (incoupled) in other experiments with 10-ps pulses. At low pump energies a small signal can be seen at $532 \mathrm{~nm}$, which we identified to be scattering of infrared pump laser radiation in the OSA. However, this background peak becomes clearly exceeded by visible supercontinuum radiation as the pump energy increases. The blue radiation centered around $480 \mathrm{~nm}$ occurs above $E_{p}=248 \mathrm{pJ}$ and is clearly visible in the 
photograph shown in Fig. 6.3(b). A small spectral feature centered at $1200 \mathrm{~nm}$ can be seen at low pulse energies as well. We found that this background feature consists of weak radiation from the pump laser, but is surpassed by supercontinuum radiation at pump energies beyond $350 \mathrm{pJ}$.

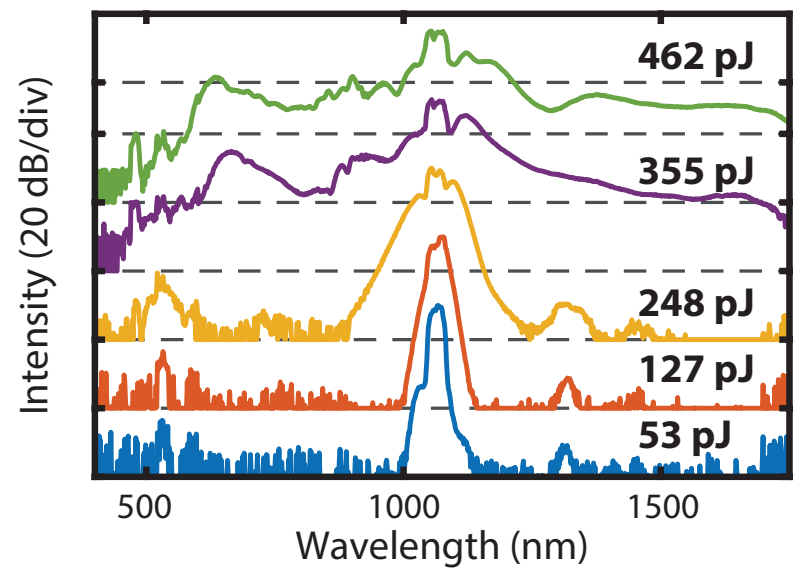

Figure 6.4: Spectral broadening of the supercontinuum in a $\mathrm{Si}_{3} \mathrm{~N}_{4}$ waveguide with $w=0.775 \mu \mathrm{m}$ as a function of the incoupled $E_{p}$. Note that for visibility a progressive offset of $20 \mathrm{~dB}$ was added.

To characterize the supercontinuum as a function of the waveguide dispersion, we injected the pump laser into waveguides of various widths. The results are shown in Fig. 6.5 for $w=0.675$ to $0.775 \mu \mathrm{m}$. The incoupled $E_{p}$ differs only slightly from waveguide to waveguide $(610 \pm 25 \mathrm{pJ})$ due to some variation in coupling losses. It can be seen that the SCG broadens with increasing $w$ at both the short wavelength side as well as the long wavelength side. This observation is in agreement with what is expected from the broadening of the anomalous dispersion range in Fig. 6.1(b).

\subsection{Numerical calculations}

For a theoretical modeling of the observed SCG, we used the multi-mode nonlinear Schrödinger equation (MM-NLSE, see Appendix) [32]. The equation describes the interaction of multiple transverse modes such as through self and cross-phase modulation, four-wave mixing, and self-steepening. We calculate that for the combination of our spatial pump beam parameters and our waveguide parameters only the fundamental mode $\left(\mathrm{TM}_{00}\right)$ and one higher order mode $\left(\mathrm{TM}_{20}\right)$ are excited and that the incoupled pulse energy is estimated to be evenly distributed among the two modes at a wavelength of $1064 \mathrm{~nm}$. The nonlinear coupling coefficient between the fundamental and higher order mode is calculated by evaluating their overlap integral. 


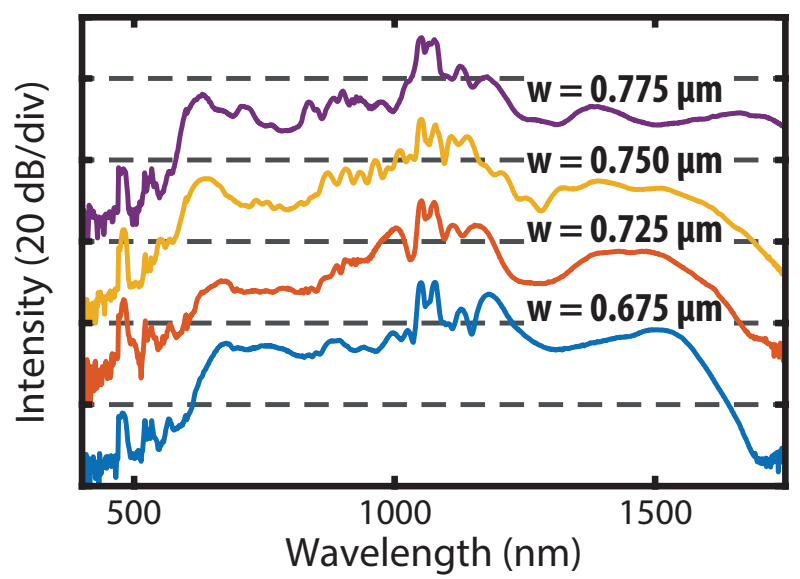

Figure 6.5: $\quad$ Supercontinuum generation with various waveguide widths, w. The obtained spectrum broadens for wider waveguides. Note that for visibility a progressive offset of $20 \mathrm{~dB}$ was added.

In the calculations, we restrict ourselves to the waveguide dimensions that provided the widest SCG as shown in Fig. 6.3(a) $(h=1.0 \mu \mathrm{m}, w=0.8 \mu \mathrm{m}$, $5.5 \mathrm{~mm}$ propagation length). The effective area of the fundamental mode is calculated to be $0.55 \mu^{2}$ for this cross section and the resulting nonlinear coefficient, $\gamma$, is $2.6 \mathrm{~W}^{-1} \mathrm{~m}^{-1}$ at a wavelength of $1064 \mathrm{~nm}$. The corresponding nonlinear length is calculated to be $0.15 \mathrm{~mm}$. In the model the dispersion of the waveguide is included up to the 18 th order. The dispersion parameter, $D$, is calculated as $60 \mathrm{ps} /(\mathrm{nm} \mathrm{km})$ at the pump wavelength and the resulting dispersion length is $0.37 \mathrm{~m}$. To include a realistic decay of the field amplitudes during propagation we take a propagation loss of $1.3 \mathrm{~dB} / \mathrm{cm}$ into account, which was measured at the pump wavelength and results in an effective length of $5.1 \mathrm{~mm}$ for the waveguide length used. Due to the huge bandwidth of the generated spectrum, it is important to consider the wavelength dependence of the effective mode area and, hence, a wavelength dependence of the nonlinear coefficient. This dependence is introduced by means of a so-called shock term [10]. We neglected the Raman effect, which appears justified in view of previous experiments with SCG in silicon nitride waveguides [19, 21], where Raman scattering did not significantly affect the obtained supercontinuum. As the input light field we take a Fourier-limited pulse with 115 fs duration and a center wavelength of $1064 \mathrm{~nm}$. Calculations show that the chirp introduced by the coupling optics can be neglected.

Figure 6.6(a) shows the spectrum of the total output (blue) calculated with a total pulse energy of $590 \mathrm{pJ}$ that is equally divided over the fundamental and the higher order mode as in the experiments. In calculations where the pulse energy in the higher order mode was varied we found that the bandwidth of the output spectrum is not sensitive to the amount of pulse energy in the higher 

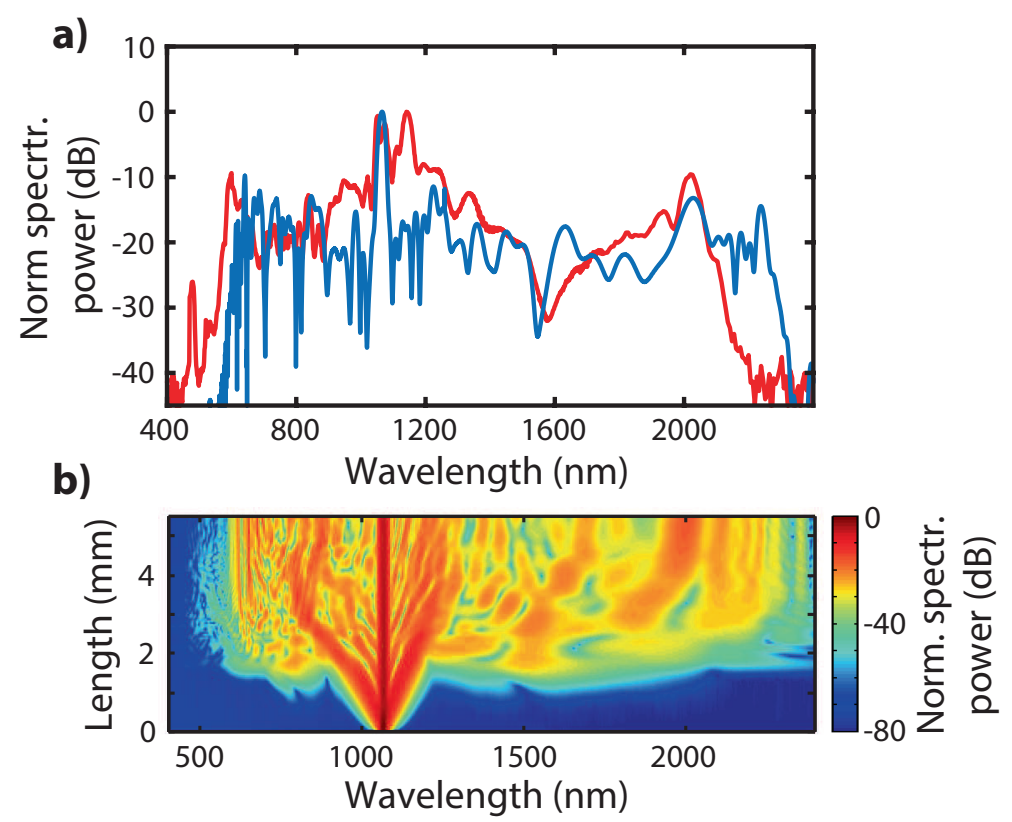

Figure 6.6: (a) Comparison of the spectrum modeled with the multimode nonlinear Schrödinger equation (blue) and the measured spectrum (red). In the calculation the pulse energy $\left(E_{p}=590 \mathrm{pJ}\right)$ and the propagation distance $(5.5 \mathrm{~mm})$ are chosen to be the same as in the experiment. (b) Spectral evolution in the numerical calculation as function of propagation distance in the waveguide. 
order mode, because the mode undergoes normal dispersion in the waveguide. For comparison the measured spectrum from Fig. 6.3(a) is shown in red. It can be seen that, besides a slight red-shift of the generated spectrum, the overall agreement between the calculated and measured supercontinuum is good. For example, both clearly show the presence of two dispersive waves. A possible reason for the red-shift might be a remaining uncertainty in the refractive index data. Furthermore, we calculated the position of the dispersive waves by their phase-matching condition [33] and found them to lie at $710 \mathrm{~nm}$ and $2080 \mathrm{~nm}$, which is in good agreement with the obtained data. Similarly, the generation of blue light at $480 \mathrm{~nm}$, which is observed in the experiments, is not explained with our current model, and a closer investigation is subject of ongoing research. In Fig. 6.6(b) the spectral broadening during the propagation is shown, calculated such as in Fig. 6.6(a), and it can be seen that already after a propagation of $2 \mathrm{~mm}$ most of the bandwidth is obtained.

\subsection{Conclusion}

In conclusion, we have shown ultra-broadband on-chip supercontinuum generation in CMOS-compatible $\mathrm{Si}_{3} \mathrm{~N}_{4}$ waveguides. When pumped at a center wavelength of $1064 \mathrm{~nm}$ with pulses of 115 fs duration, the generated spectrum ranges from the visible blue range $(470 \mathrm{~nm})$ to the infrared $(2130 \mathrm{~nm})$ and comprises a spectral bandwidth of more than $495 \mathrm{THz}$. This is, to our knowledge, the widest supercontinuum ever generated on a chip. The measurements show that the bandwidth of the supercontinuum spectrum increases with the incoupled pulse energy and with the waveguide width. A physical model based on the multi-mode nonlinear Schrödinger equation shows that only the fundamental mode contributes to the SCG into which only half of the available pump energy was coupled $(\approx 300 \mathrm{pJ})$. This suggests that a more efficient SCG might be obtained, possibly also with further increased spectral bandwidth. This might be achieved with an improved spatial control of the input coupling, e.g., by means of tapered waveguides, such that all of the available pulse energy is coupled into the fundamental mode.

The wide bandwidths of the spectra demonstrate the huge potential of integrated nonlinear optics using $\mathrm{Si}_{3} \mathrm{~N}_{4} / \mathrm{SiO}_{2}$ waveguides, provided here using the TriPleX platform [30]. The visible to infrared coverage, extending throughout most of the transparency range of $\mathrm{Si}_{3} \mathrm{~N}_{4}$ and $\mathrm{SiO}_{2}$, appears to be highly attractive for applications such as for self-referencing optical frequency combs [34] on a chip or widely tunable light sources for label-free microscopy and imaging [35] in life sciences. 


\section{Appendix}

The supercontinuum generation in the waveguides presented here was modeled by numerically solving the multi-mode generalized nonlinear Schrödinger equation (MM-NLSE) which was derived first in reference [32]. We neglect the Raman scattering contribution as it has been reported to not significantly affect SCG in silicon nitride waveguides $[19,21]$ which yields the following set of equations:

$$
\begin{aligned}
\frac{\partial A_{p}}{\partial z}= & i\left(\beta_{p}^{(0)}-\beta^{(0)}\right) A_{p}-\left(\beta_{p}^{(1)}-\beta^{(1)}\right) \frac{\delta A_{p}}{\delta t}+i \sum_{n \geq 2} \frac{\beta_{p}^{(n)}}{n !}\left(i \frac{\delta}{\delta t}\right)^{n} A_{p} \\
+ & i \frac{n_{2} \omega_{0}}{c} \sum_{l, m, n}\left\{\left(1+i \tau_{p l m n}^{(1)} \frac{\partial}{\partial t}\right) \cdot 2 Q_{p l m n}^{(1)} A_{l} A_{m} A_{n}^{*}\right. \\
& \left.+\left(1+i \tau_{p l m n}^{(2)} \frac{\partial}{\partial t}\right) \cdot Q_{p l m n}^{(2)} A_{l}^{*} A_{m} A_{n}\right\} .
\end{aligned}
$$

The evolution of the temporal envelope of the mode $p, A_{p}(z, t)$, is described in a reference frame that travels with the group velocity $\beta^{(1)}$ of the fundamental mode. The electrical field is assumed to have a frequency centered at $\omega_{0}$ and to travel with a phase velocity of $\beta_{p}^{(0)}-\beta^{(0)}$, measured relative to the one of the fundamental mode $\beta^{(0)}$. The higher order dispersion coefficients $\beta_{p}^{(n)}$ are the respective $n$-th expansion coefficients of the Taylor series of the propagation constant $\beta_{p}(\omega)$ centered around $\omega_{0}$, which accounts for the dispersion of the waveguide. The nonlinear interaction of the $p$-th mode with modes $l, m, n$ is described by the overlap integrals $Q_{p l m n}^{(1,2)}$ which can be interpreted as an effective area of the respective nonlinear interaction, $A_{\text {eff, } p l m n}=1 /\left(2 Q_{p l m n}^{(1)}+Q_{p l m n}^{(2)}\right)$. The frequency dependence of the nonlinearity is included by the shock-time constants

$$
\tau_{p l m n}^{(1,2)}=\frac{1}{\omega_{0}}+\left\{\frac{\delta}{\delta \omega} \ln Q_{p l m n}^{(1,2)}(\omega)\right\}_{\omega_{0}},
$$

taking into account the general dependence of the nonlinearity on the frequency $\omega$ as well as a correction to the shock-time by including the change in effective modal area with frequency.

\section{Acknowledgments}

This research is supported by the Dutch Technology Foundation STW, which is part of the Netherlands Organisation for Scientific Research (NWO), and which is partly funded by the Ministry of Economic Affairs. We thank W. Hoving (Anteryon BV, The Netherlands) and W. J. Mulder (Avantes BV, The Netherlands) for arranging a short-term loan of the NIR spectrometer. 


\section{Bibliography}

[1] G. Humbert, W. Wadsworth, S. Leon-Saval, J. Knight, T. Birks, P. S. J. Russell, M. Lederer, D. Kopf, K. Wiesauer, E. Breuer, and D. Stifter, "Supercontinuum generation system for optical coherence tomography based on tapered photonic crystal fibre," Optics Express 14(4), 1596 (2006).

[2] L. Yin, Q. Lin, and G. P. Agrawal, "Soliton fission and supercontinuum generation in silicon waveguides," Optics Lett. 32(4), 391 (2007).

[3] M. Wulf, D. M. Beggs, N. Rotenberg, and L. Kuipers, "Unravelling nonlinear spectral evolution using nanoscale photonic near-field point-topoint measurements," Nano Lett. 13(12), 5858-5865 (2013).

[4] J. M. Dudley, G. Genty, and B. J. Eggleton, "Harnessing and control of optical rogue waves in supercontinuum generation," Optics Express 16(6), 3644 (2008).

[5] H. Kano and H.-O. Hamaguchi, "Characterization of a supercontinuum generated from a photonic crystal fiber and its application to coherent Raman spectroscopy," Optics Lett. 28(23), 2360 (2003).

[6] J. T. Woodward, A. W. Smith, C. A. Jenkins, C. Lin, S. W. Brown, and K. R. Lykke, "Supercontinuum sources for metrology," Metrologia 46(4), S277 (2009).

[7] F. Silva, D. Austin, A. Thai, M. Baudisch, M. Hemmer, D. Faccio, A. Couairon, and J. Biegert, "Multi-octave supercontinuum generation from mid-infrared filamentation in a bulk crystal," Nature Commun. 3, 807 (2012).

[8] T. A. Birks, W. J. Wadsworth, and P. S. J. Russell, "Supercontinuum generation in tapered fibers," Optics Lett. 25(19), 1415 (2000).

[9] A. Marandi, C. W. Rudy, V. G. Plotnichenko, E. M. Dianov, K. L. Vodopyanov, and R. L. Byer, "Mid-infrared supercontinuum generation in tapered chalcogenide fiber for producing octave-spanning frequency comb around 3 нm," Optics Express 20(22), 24218-25 (2012).

[10] J. M. Dudley and S. Coen, "Supercontinuum generation in photonic crystal fiber," Rev. Mod. Phys. 78(4), 1135-1184 (2006). 
[11] M. Klimczak, G. Stepniewski, H. Bookey, A. Szolno, R. Stepien, D. Pysz, A. Kar, A. Waddie, M. R. Taghizadeh, and R. Buczynski, "Broadband infrared supercontinuum generation in hexagonal-lattice tellurite photonic crystal fiber with dispersion optimized for pumping near $1560 \mathrm{~nm}$," Optics Lett. 38(22), 4679-82 (2013).

[12] X. Jiang, N. Y. Joly, M. A. Finger, F. Babic, G. K. L. Wong, J. C. Travers, and P. S. J. Russell, "Deep-ultraviolet to mid-infrared supercontinuum generated in solid-core ZBLAN photonic crystal fibre," Nature Photon. 9(2), 133-139 (2015).

[13] C. R. Phillips, C. Langrock, J. S. Pelc, M. M. Fejer, J. Jiang, M. E. Fermann, and I. Hartl, "Supercontinuum generation in quasi-phase-matched $\mathrm{LiNbO}_{3}$ waveguide pumped by a Tm-doped fiber laser system," Optics Lett. 36(19), 3912-4 (2011).

[14] B. Rangarajan, A. Y. Kovalgin, K. Wörhoff, and J. Schmitz, "Lowtemperature deposition of high-quality silicon oxynitride films for CMOSintegrated optics," Optics Lett. 38(6), 941-3 (2013).

[15] F. Leo, S.-P. Gorza, J. Safioui, P. Kockaert, S. Coen, U. Dave, B. Kuyken, and G. Roelkens, "Dispersive wave emission and supercontinuum generation in a silicon wire waveguide pumped around the $1550 \mathrm{~nm}$ telecommunication wavelength," Optics Lett. 39(12), 3623-6 (2014).

[16] B. Kuyken, T. Ideguchi, S. Holzner, M. Yan, T. W. Hänsch, J. Van Campenhout, P. Verheyen, S. Coen, F. Leo, R. Baets, G. Roelkens, and N. Picqué, "An octave-spanning mid-infrared frequency comb generated in a silicon nanophotonic wire waveguide," Nature Commun. 6, 6310 (2015).

[17] D. Y. Oh, D. Sell, H. Lee, K. Y. Yang, S. A. Diddams, and K. J. Vahala, "Supercontinuum generation in an on-chip silica waveguide," Opitcs Lett. 39(4), 1046-8 (2014).

[18] D. Duchesne, M. Peccianti, M. R. E. Lamont, M. Ferrera, L. Razzari, F. Légaré, R. Morandotti, S. Chu, B. E. Little, and D. J. Moss, "Supercontinuum generation in a high index doped silica glass spiral waveguide," Optics Express 18(2), 923-30 (2010).

[19] R. Halir, Y. Okawachi, J. S. Levy, M. A. Foster, M. Lipson, and A. L. Gaeta, "Ultrabroadband supercontinuum generation in a CMOScompatible platform," Opitcs Lett. 37(10), 1685-7 (2012).

[20] J. M. Chavez Boggio, D. Bodenmüller, T. Fremberg, R. Haynes, M. M. Roth, R. Eisermann, M. Lisker, L. Zimmermann, and M. Böhm, "Dispersion engineered silicon nitride waveguides by geometrical and refractiveindex optimization," J. Opt. Soc. Am. B 31(11), 2846 (2014). 
[21] H. Zhao, B. Kuyken, S. Clemmen, F. Leo, A. Subramanian, A. Dhakal, P. Helin, S. Severi, E. Brainis, G. Roelkens, and R. Baets, "Visible-to-nearinfrared octave spanning supercontinuum generation in a silicon nitride waveguide," Optics Lett. 40(10), 2177-80 (2015).

[22] A. Gondarenko, J. S. Levy, and M. Lipson, "High confinement micron-scale silicon nitride high Q ring resonator," Optics Express 17(14), 11366-70 (2009).

[23] J. F. Bauters, M. J. R. Heck, D. John, D. Dai, M.-c. Tien, J. S. Barton, A. Leinse, R. G. Heideman, D. J. Blumenthal, and J. E. Bowers, "Ultralow-loss high-aspect-ratio $\mathrm{Si}_{3} \mathrm{~N}_{4}$ waveguides," Optics Express 19(4), 316374 (2011).

[24] K. Ikeda, R. E. Saperstein, N. Alic, and Y. Fainman, "Thermal and Kerr nonlinear properties of plasma-deposited silicon nitride/silicon dioxide waveguides," Optics Express 16(17), 12987-94 (2008).

[25] C. G. H. Roeloffzen, L. Zhuang, C. Taddei, A. Leinse, R. G. Heideman, P. W. L. van Dijk, R. M. Oldenbeuving, D. A. I. Marpaung, M. Burla, and K.-J. Boller, "Silicon nitride microwave photonic circuits," Optics Express 21(19), 22937-61 (2013).

[26] E. S. Hosseini, P. Purnawirman, J. D. B. Bradley, J. Sun, G. Leake, T. N. Adam, D. D. Coolbaugh, and M. R. Watts, "CMOS-compatible $75 \mathrm{~mW}$ erbium-doped distributed feedback laser," Optics Lett. 39(11), 3106-9 (2014).

[27] L. Zhang, Y. Yan, Y. Yue, Q. Lin, O. Painter, R. G. Beausoleil, and A. E. Willner, "On-chip two-octave supercontinuum generation by enhancing self-steepening of optical pulses," Optics Express 19(12), 11584-90 (2011).

[28] J. Riemensberger, K. Hartinger, T. Herr, V. Brasch, R. Holzwarth, and T. J. Kippenberg, "Dispersion engineering of thick high-Q silicon nitride ring-resonators via atomic layer deposition," Optics Express 20(25), 27661-9 (2012).

[29] J. P. Epping, M. Hoekman, R. Mateman, A. Leinse, R. G. Heideman, A. van Rees, P. J. van der Slot, C. J. Lee, and K.-J. Boller, "High confinement, high yield $\mathrm{Si}_{3} \mathrm{~N}_{4}$ waveguides for nonlinear optical applications," Optics Express 23(2), 642 (2015).

[30] K. Wörhoff, R. G. Heideman, A. Leinse, and M. Hoekman, "TriPleX: a versatile dielectric photonic platform," Adv. Opt. Techn. 4(2), 189-207 (2015).

[31] A. B. Fallahkhair, K. S. Li, and T. E. Murphy, "Vector finite difference modesolver for anisotropic dielectric waveguides," J. Lightwave Technol. 26(11), 1423-1431 (2008). 
[32] F. Poletti and P. Horak, "Description of ultrashort pulse propagation in multimode optical fibers," J. Opt. Soc. Am. B 25(10), 1645-54 (2008).

[33] G. P. Agrawal, Nonlinear Fiber Optics, 4th ed., (Academic, 2007).

[34] D. J. Jones, S. A. Diddams, J. K. Ranka, A. Stentz, R. S. Windeler, J. L. Hall, and S. T. Cundiff, "Carrier-envelope phase control of femtosecond mode-locked lasers and direct optical frequency synthesis," Science 288(5466), 635-639 (2000).

[35] J. P. Epping, M. Kues, P. J. M. van der Slot, C. J. Lee, C. Fallnich, and K.-J. Boller, "Integrated CARS source based on seeded four-wave mixing in silicon nitride," Optics Express 21(26), 32123-9 (2013). 
Conclusion and outlook 
In conclusion we investigated, in this thesis, the on-chip nonlinear frequency conversion of optical wavelengths using silicon nitride waveguides. Two nonlinear conversion schemes were considered: seeded four-wave mixing, which was presented in Chapter 3, and supercontinuum generation, presented in Chapters 5 and 6 . In Chapter 3 we presented numerical calculations on seeded four-wave mixing in $\mathrm{Si}_{3} \mathrm{~N}_{4}$ waveguides, which is the first work ever to consider a light source for coherent anti-Stokes Raman scattering (CARS) based on integrated photonics. The compatibility of some integrated platforms, especially the $\mathrm{Si}_{3} \mathrm{~N}_{4} / \mathrm{SiO}_{2}$ platform [1], with microfluidic channels enables potential applications for on-chip CARS spectroscopy.

Both conversion schemes, four-wave mixing as well as supercontinuum generation, require waveguides with a large core area $\left(\approx 1 \mu \mathrm{m}^{2}\right)$ to obtain the dispersion required for phase-matched nonlinear frequency conversion. A novel fabrication technique for manufacturing large-core $\mathrm{Si}_{3} \mathrm{~N}_{4}$ waveguides was presented in Chapter 4. The main advantage of this novel technique is the ability to manufacture crack-free $\mathrm{Si}_{3} \mathrm{~N}_{4}$ waveguides with sufficient thickness to phase match nonlinear optical processes, while simultaneously realizing a high device yield. To demonstrate that such waveguides can be dispersion engineered and, i.e., allow for phase matching for nonlinear frequency conversion, we experimentally investigated supercontinuum generation in these waveguides using two different pump wavelengths (see Chapter 5 and 6).

For a pump wavelength of $1560 \mathrm{~nm}$ (see Chapter 5), the waveguide was dispersion engineered to have a zero-dispersion wavelength just above $1600 \mathrm{~nm}$, such that the pump wavelength experiences anomalous dispersion. The generated supercontinuum spanned more than $700 \mathrm{~nm}$ (at $-30 \mathrm{~dB}$ ), limited by the available pump energy. Theoretical modeling showed an exceptionally good agreement with the measured spectrum, and that an octave-spanning supercontinuum is possible if the pump energy is increased. The pulse-to-pulse coherence was calculated for this case and we found that the supercontinuum was fully coherent over its bandwidth $(-30 \mathrm{~dB})$, showing that $\mathrm{Si}_{3} \mathrm{~N}_{4}$ waveguides could be used to generate an optical frequency comb. For a pump wavelength of $1064 \mathrm{~nm}$ (Chapter 6), the waveguide was designed to have a zero-dispersion wavelength just below $1000 \mathrm{~nm}$ to have, again, anomalous dispersion for the pump wavelength. At this pump wavelength, the generated supercontinuum spanned an ultrabroad bandwidth of nearly $500 \mathrm{THz}$ covering nearly the whole transparency window of the $\mathrm{Si}_{3} \mathrm{~N}_{4} / \mathrm{SiO}_{2}$ waveguide.

To conclude, we have successfully demonstrated nonlinear frequency conversion in $\mathrm{Si}_{3} \mathrm{~N}_{4} / \mathrm{SiO}_{2}$ waveguides, which shows promise for versatile integrated light sources that are based on a novel high-yield manufacturing process. For example, the demonstrated supercontinuum generation enables applications like optical frequency metrology and, possibly, optical clocks based on integrated waveguides. On the other hand, due to the extension of the spectrum into the visible wavelength range, which is required for some spectroscopic applications, e.g., in the life sciences. Furthermore, the compatibility with microfluidic channels enables highly integrated lab-on-a-chip applications. 


\section{Outlook}

The waveguide fabrication, as described in Chapter 3, can be improved for optimal nonlinear frequency conversion. The most obvious point is to avoid the gap that appeared between the $\mathrm{Si}_{3} \mathrm{~N}_{4}$ layers within the trench. In order to avoid the formation of a gap, but, at the same time provide a similar dispersion and mode area, the aspect ratio of the waveguide core has to be changed to wider cores with less height. Alternatively, the sidewalls could be tapered more strongly by etching the trenches under a different angle. Furthermore, the waveguide design can be improved by including mode converters at the input and output facets of the waveguide chips. Using mode converters would have two main advantages for more efficient nonlinear frequency conversion. Firstly, the incoupling loss (8-9 $\mathrm{dB}$ for the waveguides used in this thesis) could be dramatically decreased. Fiber-to-chip coupling losses as low as $0.5 \mathrm{~dB}$ [1] have been demonstrated. Secondly, the excitation of higher order modes can be suppressed to a greater degree. The higher order modes of the waveguides presented in this thesis experience normal dispersion and, as a consequence, do not contribute to nonlinear frequency conversion (see Chapter 6). Therefore, it is of importance to use mode converters to ensure efficient coupling into the fundamental mode of the waveguide.

The bandwidth for nonlinear frequency conversion using $\mathrm{Si}_{3} \mathrm{~N}_{4} / \mathrm{SiO}_{2}$ is limited at the long wavelength side to about $2400 \mathrm{~nm}$ in $\mathrm{Si}_{3} \mathrm{~N}_{4} / \mathrm{SiO}_{2}$, due to increasing absorption at longer wavelengths by the cladding material $\left(\mathrm{SiO}_{2}\right)$. For shorter wavelengths, nonlinear frequency conversion is limited by the material dispersion of the $\mathrm{Si}_{3} \mathrm{~N}_{4}$ core. The material dispersion of $\mathrm{Si}_{3} \mathrm{~N}_{4}$ becomes dominant towards shorter wavelengths because $\mathrm{Si}_{3} \mathrm{~N}_{4}$ has a lower energy band gap than $\mathrm{SiO}_{2}$, and the resulting increase in refractive index cannot be compensated by waveguide dispersion. Hence, nonlinear processes cannot be phase matched anymore. Nonlinear frequency conversion at short wavelengths may also be limited by two-photon absorption, which is possible for wavelength shorter than $600 \mathrm{~nm}[2]$. Though, we should note that we observed no evidence of two-photon absorption at the maximum incoupled powers that we achieved in this thesis.

To improve the dispersion engineering it is promising to add another degree of freedom to the $\mathrm{Si}_{3} \mathrm{~N}_{4} / \mathrm{SiO}_{2}$ platform by using a different material as top cladding. Including a third material in the cladding can realize more efficient nonlinear frequency conversion by flattening the dispersion. Alternatively, a wider bandwidth of conversion can be achieved by broadening the anomalous dispersion range of the waveguides. Especially, using the fabrication technique described in Chapter 4, it would be possible to deposit a third material on top of the planarized wafer instead of $\mathrm{SiO}_{2}$. Similar dispersion engineering approaches were reported for conventionally processed silicon nitride waveguides $[3,4]$. The added material could be deposited by, e.g., atomic layer deposition [3], chemical vapor deposition [4], or, perhaps, pulsed laser deposition [5], which would also allow the growth of crystalline layers. 
Furthermore, to be more energy efficient, as is desirable for some applications in telecommunication, it can be advantageous to include materials with a nonlinearity higher than that of $\mathrm{Si}_{3} \mathrm{~N}_{4}$. For example, very efficient supercontinuum generation, pumped at the telecommunication C-band, has been achieved in highly nonlinaer materials, e.g., such as hydrogenated amorphous silicon (a-Si:H) [6] or InGaP [7]. Amorphous silicon has a lower two-photon absorption than crystalline silicon, while InGaP does not suffer from two-photon absorption at $1550 \mathrm{~nm}$. Furthermore, both InGaP and a-Si:H platforms have been reported to generate supercontinuum spectra with a high degree of coherence $[7,8]$. In general, however, these materials have a higher linear loss than $\mathrm{Si}_{3} \mathrm{~N}_{4}$ waveguides, which will limit the propagation length in such waveguides. In addition, the refractive indices of $\mathrm{InGaP}$ and a-Si:H are larger than that of $\mathrm{Si}_{3} \mathrm{~N}_{4}$, meaning that it cannot be used as the outer cladding material. An easy way to add such materials to $\mathrm{Si}_{3} \mathrm{~N}_{4} / \mathrm{SiO}_{2}$ waveguides would be to deposit a thin layer on top of the waveguide before adding a $\mathrm{SiO}_{2}$ top cladding (see Chapter 4). A second method would be to deposit the material while the trench is being filled so that part of the core consists of a more nonlinear material. An advantage of both methods is that the remainder of the photonic circuitry can be realized using standard low-loss $\mathrm{Si}_{3} \mathrm{~N}_{4} / \mathrm{SiO}_{2}$ waveguides.

Another possible application, which we have numerically investigated, is seeded four-wave mixing in $\mathrm{Si}_{3} \mathrm{~N}_{4} / \mathrm{SiO}_{2}$ waveguides for use as an integrated light source for CARS (see Chapter 3). A next logical step would be to experimentally demonstrate such a light source. In Chapter 6 we have already shown that the dispersion needed to phase match nonlinear frequency conversion over a broad bandwidth is feasible using $\mathrm{Si}_{3} \mathrm{~N}_{4}$ waveguides and, furthermore, the same pump wavelength of $1064 \mathrm{~nm}$ (as considered in Chapter 3) was used. However, the damage threshold of $\mathrm{Si}_{3} \mathrm{~N}_{4}$ waveguides for picosecond pulses at a center wavelength of $1064 \mathrm{~nm}$ (as reported in Chapter 6) could be a crucial point. With this damage threshold in mind, pulses with a duration shorter than the 10 ps used in the numeric modeling (as in Chapter 3) should be considered. This would have the advantage of a higher peak intensity and hence a higher four-wave mixing gain. However, this comes at the cost of spectral resolution in a possible CARS experiment, as these pulses have a broader input spectrum. Another approach might be the use of a pump laser with a longer wavelength, such as $1550 \mu \mathrm{m}$, as the damage threshold is expected to be higher at this wavelength.

So far we only numerically calculated the pulse-to-pulse coherence of the obtained supercontinuum spectra in Chapter 5. However, we have not yet experimentally validated the coherence properties of the experimentally observed supercontinuum spectra. To validate the pulse-to-pulse coherence, experiments, such as beat-note measurements $[9,10]$ with a single frequency laser or spectral interferometery [11] have to be performed. Furthermore, to show optical frequency combs generated on a chip it would be interesting to implement selfreferencing by an $f-2 f$ or a $2 f-3 f$ interferometer. Of special interest is that dispersion engineering can also be used to enhance second harmonic [12] and 
third harmonic $[12,13]$ generation via phase matching between higher order modes, which would enable chip-based $f-2 f$ or $2 f-3 f$ interferometers.

In order to accurately engineer nonlinear processes in more complex optical waveguide circuits in the future, it would be important to precisely measure the material properties of stoichiometric $\mathrm{Si}_{3} \mathrm{~N}_{4}$, i.e., the Kerr index, $n_{2}$, and the Raman effect of $\mathrm{Si}_{3} \mathrm{~N}_{4}$ waveguides. The Kerr index that is used throughout this thesis is $n_{2}=2.4 \cdot 10^{-19} \mathrm{~m}^{2} \mathrm{~W}^{-1}[14]$, and was measured using waveguides with silicon nitride deposited by plasma-enhanced chemical vapor deposition, whose $n_{2}$ might be slightly different from stoichiometric silicon nitride deposited by low-pressure chemical vapor deposition. Furthermore, the Raman effect is often ignored in silicon nitride waveguides as it was reported that it does not have a significant impact on the resulting supercontinuum spectrum [15], however, it still might have an impact on nonlinear frequency conversion, especially on the coherence properties of the supercontinuum spectrum. The inclusion of the actual Raman effect of $\mathrm{Si}_{3} \mathrm{~N}_{4}$, i.e., the Raman response function, $h_{R}$, and its contribution, $f_{R}$, would allow for higher precision numerical modeling.

The large-core $\mathrm{Si}_{3} \mathrm{~N}_{4}$ waveguides considered in this thesis are multi-mode. The higher order modes experience normal dispersion and, therefore, do not contribute to the supercontinuum generation studied in this work (Chapter 6).

As mentioned above an excitation of higher order modes should be avoided for the purpose of supercontinuum generation as they do not contribute to the spectral broadening. However, it has recently been shown that higher order modes in integrated waveguides can be exploited in other nonlinear optical processes, e.g., for all-optical switching. In a numerical study by Hellwig et al. [16], it was shown that nonlinear mode conversion with a conversion efficiency of up to $90 \%$ could be harnessed in silicon nitride waveguides at moderate pulse energies below $1 \mathrm{~nJ}$. Such pulse energies are promising, because the power needed for such switching schemes can be greatly decreased by using integrated waveguides in place of optical fibers. For example, mode conversion could be used to achieve ultra-fast switching speeds in future telecommunication networks, therefore, it would be promising to selectively excite certain waveguide modes and even convert or switch between various waveguide modes. 



\section{Bibliography}

[1] K. Wörhoff, R. G. Heideman, A. Leinse, and M. Hoekman, "TriPleX: a versatile dielectric photonic platform," Adv. Opt. Techn. 4, 189 (2015).

[2] E. S. Hosseini, S. Yegnanarayanan, A. H. Atabaki, M. Soltani, and A. Adibi, "High quality planar silicon nitride microdisk resonators for integrated photonics in the visible wavelength range," Optics Express 17(17), 14545 (2009).

[3] J. Riemensberger, K. Hartinger, T. Herr, V. Brasch, R. Holzwarth, and T. J. Kippenberg, "Dispersion engineering of thick high-Q silicon nitride ring-resonators via atomic layer deposition," Optics Express 20(25), 27661-9 (2012).

[4] J. M. Chavez Boggio, D. Bodenmüller, T. Fremberg, R. Haynes, M. M. Roth, R. Eisermann, M. Lisker, L. Zimmermann, and M. Böhm, "Dispersion engineered silicon nitride waveguides by geometrical and refractiveindex optimization," J. Opt. Soc. Am. B 31(11), 2846 (2014).

[5] K. E. Youden, T. Grevatt, R. W. Eason, H. N. Rutt, R. S. Deol, and G. Wylangowski, "Pulsed laser deposition of GaLaS chalcogenide glass thin film optical waveguides," Appl. Phys. Lett. 63, 1601 (1993).

[6] J. Safioui, F. Leo, B. Kuyken, S.-P. Gorza, S. K. Selvaraja, R. Baets, P. Emplit, G. Roelkens, and S. Massar, "Supercontinuum generation in hydrogenated amorphous silicon waveguides at telecommunication wavelengths," Optics Express 22(3), 3089 (2014).

[7] U. Dave, C. Ciret, S.-P. Gorza, S. Combrié, A. De Rossi, F. Raineri, G. Roelkens, and B. Kuyken "Dispersive wave based octave spanning supercontinuum generation in InGaP membrane waveguides on a silicon substrate," Optics Lett. 40(15), 3584 (2015).

[8] F. Leo, J. Safioui, B. Kuyken, G. Roelkens, and S.-P. Gorza, "Generation of coherent supercontinuum in a-Si:H waveguides: experiment and modeling based on measured dispersion profile," Optics Express 22(23), 28997 (2014).

[9] A. Ruehl, M. J. Martin, K. C. Cossel, L. Chen, H. McKay, B. Thomas, C. Benko, L. Dong, J. M. Dudley, M. E. Fermann, I. Hartl, and J. Ye, 
"Ultrabroadband coherent supercontinuum frequency comb," Phys. Rev. A 84, 3 (2011).

[10] B. Kuyken, T. Ideguchi, S. Holzner, M. Yan, T. W. Hänsch, J. Van Campenhout, P. Verheyen, S. Coen, F. Leo, R. Baets, G. Roelkens, and N. Picqué, "An octave-spanning mid-infrared frequency comb generated in a silicon nanophotonic wire waveguide," Nature Commun. 6, 6310 (2015).

[11] F. Lu and W. H. Knox, "Generation of a broadband continuum with high spectral coherence in tapered single-mode optical fibers," Optics Express $\mathbf{1 2}(2), 347$ (2004).

[12] J. S. Levy, M. A. Foster, A. L. Gaeta, and M. Lipson, "Harmonic generation in silicon nitride ring resonators," Optics Express 19(12), 1141511421 (2011).

[13] J. P. Epping, T. Hellwig, R. Mateman, A. van Rees, M. Hoekman, A. Leinse, R. G. Heideman, P. J. M. van der Slot, C. J. Lee, C. Fallnich, and K.-J. Boller, "Simultaneous supercontinuum and third harmonic generation in $\mathrm{Si}_{3} \mathrm{~N}_{4}$ waveguides," CLEO/Europe-EQEC, CK-5.4 (2015).

[14] K. Ikeda, R. E. Saperstein, N. Alic, and Y. Fainman, "Thermal and Kerr nonlinear properties of plasma-deposited silicon nitride/silicon dioxide waveguides," Optics Express 16, 12987 (2008).

[15] H. Zhao, B. Kuyken, S. Clemmen, F. Leo, A. Subramanian, A. Dhakal, P. Helin, S. Severi, E. Brainis, G. Roelkens, and R. Baets, "Visible-to-nearinfrared octave spanning supercontinuum generation in a silicon nitride waveguide," Optics Lett. 40(10), 2177-80 (2015).

[16] T. Hellwig, J. P. Epping, M. Schnack, K.-J. Boller, and C. Fallnich, "Ultrafast, low-power, all-optical switching via birefringent phase-matched transverse mode conversion in integrated waveguides," Optics Express 23(15), 19189-201 (2015). 


\section{Summary}

In this thesis we investigate nonlinear optical frequency conversion in integrated stoichiometric silicon nitride $\left(\mathrm{Si}_{3} \mathrm{~N}_{4}\right)$ waveguides. Using integrated waveguides for nonlinear frequency conversion is of particular interest as it allows for nonlinear frequency conversion at relatively low pump energies and a small devise footprint compared to fiber-based devices. Specifically, integrated waveguides that offer compatibility with well-established and reliable fabrication technologies, such as CMOS, are promising, as they can be easier brought into low-cost mass production to obtain highly integrated photonic circuits. Of the CMOS compatible integrated platforms, $\mathrm{Si}_{3} \mathrm{~N}_{4}$ waveguides offers a moderate nonlinearity (between those of $\mathrm{SiO}_{2}$ and $\mathrm{Si}$ ), while in the near-infrared $\mathrm{Si}_{3} \mathrm{~N}_{4}$ does not suffer from nonlinear losses, i.e., two-photon absorption. An additional advantage of $\mathrm{Si}_{3} \mathrm{~N}_{4}$ waveguides is the broad transparency window, ranging from $400 \mathrm{~nm}$ to $2400 \mathrm{~nm}$, which enables nonlinear frequency conversion over a broad bandwidth, and potentially low propagation losses.

In Chapter 2 we introduce the generalized nonlinear Schrödinger equation (GNLSE) that forms the basis for modeling nonlinear pulse propagation in integrated waveguides. In Chapter 3, we use the GNLSE to theoretically investigate an integrated nonlinear light source for coherent anti-Stokes Raman scattering (CARS), based on $\mathrm{Si}_{3} \mathrm{~N}_{4}$ waveguides. By realizing such an integrated light source, CARS spectroscopy can, e.g., be performed in a lab-on-a-chip setup with a tiny device footprint compared to conventional CARS setups. To obtain temporally synchronized signal and idler pulses that are suitable in both bandwidth and peak power for CARS spectroscopy and microscopy, we consider four-wave mixing of a strong pump pulse. In order to match the bandwidth of the generated pulses with the bandwidth of typical vibrational transitions, we consider a pulse with a pulse duration of $10 \mathrm{ps}$ and a fixed center wavelength of $1064 \mathrm{~nm}$. Furthermore, to match the difference frequencies of the generated signal and idler pulses to various vibrational transitions, we additionally consider a wavelength-tunable continuous wave laser to seed the four-wave mixing process. We find that the calculated input pump power needed for nonlinear wavelength generation is more than one order of magnitude lower than in previously reported approaches based on optical fibers. The tuning range of the wavelength conversion was calculated to be from $1418 \mathrm{~nm}$ to $1518 \mathrm{~nm}$ (idler) and from $788 \mathrm{~nm}$ to $857 \mathrm{~nm}$ (signal), which corresponds to a coverage of vibrational transitions from $2350 \mathrm{~cm}^{-1}$ to $2810 \mathrm{~cm}^{-1}$. A maximum conversion efficiency of $19.1 \%$ at a peak pump power of $300 \mathrm{~W}(\approx 2.8 \mathrm{~nJ})$ is predicted. 
To phase match nonlinear optical processes, such as four-wave mixing (see Chapter 3) and supercontinuum generation (see Chapters 5 and 6), it is important that the pump wavelength is located close to the zero dispersion wavelength (ZDW). To engineer the dispersion of $\mathrm{Si}_{3} \mathrm{~N}_{4} / \mathrm{SiO}_{2}$ waveguides such that a ZDW wavelength occurs near a wavelength for which laser sources are readily available, $\mathrm{Si}_{3} \mathrm{~N}_{4}$ layer thicknesses of more than $0.6 \mu \mathrm{m}$ are needed. However, layer thicknesses of this size are challenging with conventional fabrication methods of $\mathrm{Si}_{3} \mathrm{~N}_{4}$ waveguides due to accumulating tensile stress in $\mathrm{Si}_{3} \mathrm{~N}_{4}$ layer when using low-pressure chemical vapor deposition (LPCVD), which produces $\mathrm{Si}_{3} \mathrm{~N}_{4}$ with the best optical properties. The accumulating stress in the $\mathrm{Si}_{3} \mathrm{~N}_{4}$ layer ultimately results in the formation of cracks for thickness of more than $0.4 \mu \mathrm{m}$, which severely limits the device yield. In Chapter 4, we present a novel fabrication technique for $\mathrm{Si}_{3} \mathrm{~N}_{4} / \mathrm{SiO}_{2}$ waveguides with thicknesses up to $0.9 \mu \mathrm{m}$, which is suitable for nonlinear optical applications. The increase in layer thickness is achieved by first etching trenches in a thermally oxidized silicon substrate and, in a second step, filling the trenches with $\mathrm{Si}_{3} \mathrm{~N}_{4}$. Due to the congruent growth of LPCVD $\mathrm{Si}_{3} \mathrm{~N}_{4}$ the layer grows equally from the bottom and sidewalls of the trench until the $\mathrm{Si}_{3} \mathrm{~N}_{4}$ layers from the sidewalls merge and uniformly fill the trench. To obtain the final waveguide core, the $\mathrm{Si}_{3} \mathrm{~N}_{4}$

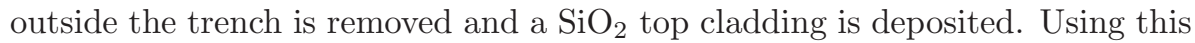
technique no stress-induced cracks in the $\mathrm{Si}_{3} \mathrm{~N}_{4}$ layer were observed, resulting in a high yield of devices on the wafer. The propagation losses of the obtained waveguides were measured to be as low as $0.4 \mathrm{~dB} / \mathrm{cm}$ at a wavelength of around $1550 \mathrm{~nm}$, allowing for a long interaction length. In addition, the bending losses of the waveguides were calculated to be low due to the high confinement in the waveguide core, which also allows for a high density of waveguide circuits on processed wafers.

Using the waveguides fabricated by the process described in Chapter 4, we report on broadband supercontinuum generation in Chapters 5 and 6 , while driven by ultrashort pump pulses with center wavelengths of $1560 \mathrm{~nm}$ and $1064 \mathrm{~nm}$, respectively. In Chapter 5 we used a mode-locked erbium-doped fiber laser with a pulse duration of $65 \mathrm{fs}$ and a pulse energy of $43 \mathrm{pJ}$ to generate a supercontinuum spectrum with a bandwidth of more than $700 \mathrm{~nm}$ at a $30 \mathrm{~dB}$ level when pumped in the anomalous group velocity dispersion regime. We achieved excellent agreement between the experimentally measured and calculated spectrum, which again was obtained by using a model based on the GNLSE. Extending the pump pulse energy to beyond the experimental value, but still far below the damage threshold of the waveguides, detailed numerical calculations show that an octave-spanning supercontinuum with a high degree of coherence over the entire generated bandwidth can be obtained at pulse energies as small as $50 \mathrm{pJ}$. These calculations show that large-core $\mathrm{Si}_{3} \mathrm{~N}_{4}$ waveguides have great potential for on-chip generation of frequency combs and many other applications.

The generated supercontinuum spectrum reported in Chapter 5 spans from $1225 \mathrm{~nm}$ to $1960 \mathrm{~nm}$, when it is pumped near the longer wavelength of the 
two ZDWs. To extend supercontinuum generation to shorter wavelengths - as is required for some applications in the life sciences - the supercontinuum has to be pumped close to the ZDW on the shorter wavelength side. As reported in Chapter 6, we used a mode-locked ytterbium-doped fiber laser with a center wavelength of $1064 \mathrm{~nm}$ and a pulse duration of $115 \mathrm{fs}$ to generate an ultrabroadband supercontinuum including most of the visible spectrum. At an incoupled pulse energy of $590 \mathrm{pJ}$ the generated spectrum extends through the visible (from $470 \mathrm{~nm}$ ) to the infrared spectral range $(2130 \mathrm{~nm}$ ) at a $-30 \mathrm{~dB}$ level, comprising a spectral bandwidth wider than $495 \mathrm{THz}$, which is today the widest supercontinuum spectrum generated on a chip. To improve the accuracy of our numerical model of supercontinuum generation, we extended the model used in Chapter 5 to include higher order spatial modes, since, at a pump wavelength of $1064 \mathrm{~nm}$, the waveguides support more than the fundamental spatial mode. Furthermore, by using this model, we observed that after $2 \mathrm{~mm}$ of propagation most of the bandwidth of the supercontinuum is already obtained, which shows that, potentially, very small and efficient devices for nonlinear wavelength conversion in silicon nitride waveguides can be achieved.

The results presented in this thesis show that $\mathrm{Si}_{3} \mathrm{~N}_{4} / \mathrm{SiO}_{2}$ waveguides hold a great potential for on-chip nonlinear frequency conversion that lead to highly integrated, versatile light sources with a small footprint that can be utilized for numerous applications. 



\section{Nederlandse Samenvatting}

In dit proefschrift onderzoeken we niet-lineaire optische frequentieomzetting in geïntegreerde, stoichiometrische siliciumnitride $\left(\mathrm{Si}_{3} \mathrm{~N}_{4}\right)$ golfgeleiders. Het gebruik van geïntegreerde golfgeleiders voor niet-lineaire frequentieomzetting is zeer interessant omdat deze omzetting al mogelijk is bij relatief lage pompenergieën, en bovendien resulteert in compacte apparaten in vergelijking met vergelikbare apparatuur gebaseerd op glasvezels. Indien de geïntegreerde golfgeleiders bovendien compatibel zijn met wijdverspreide en betrouwbare productietechnologieën, zoals voor CMOS, is goedkope massaproductie van complexe, geïntegreerde fotonische circuits mogelijk. Een van de geïntegreerde platvorms dat met CMOS compatibel is wordt gevormd door $\mathrm{Si}_{3} \mathrm{~N}_{4}$-golfgeleiders. Dit platform biedt een redelijke niet-lineairiteit (tussen die van $\mathrm{SiO}_{2}$ en $\mathrm{Si}$ ) en een bijkomend voordeel is de afwezigheid van niet-lineaire verliezen, zoals twee-fotonabsorptie, in het nabij-infrarood. Andere voordelen van $\mathrm{Si}_{3} \mathrm{~N}_{4}$-golfgeleiders zijn het brede transmissievenster, lopend van $400 \mathrm{~nm}$ tot $2400 \mathrm{~nm}$, en lage voortplantingsverliezen. Het brede transmissievenster maakt in principe niet-lineaire frequentieomzetting mogelijk met een eveneens brede bandbreedte.

In hoofdstuk 2 introduceren we de gegeneraliseerde niet-lineaire Schrödingervergelijking (GNLSE), die de basis vormt voor het modelleren van niet-lineaire voortplanting van een optische puls in gë̈ntegreerde golfgeleiders. In hoofdstuk 3 gebruiken we de GNLSE voor een theoretische studie naar een geïntegreerde, niet-lineaire lichtbron gebaseerd op $\mathrm{Si}_{3} \mathrm{~N}_{4}$-golfgeleiders voor coherente, anti-Stokes Ramanverstrooiing (CARS). Door het realiseren van zon geïntegreerde lichtbron kan CARS-spectroscopie bijvoorbeeld uitgevoerd worden in een, in vergelijking met conventionele CARS-opstellingen, zeer compacte lab-on-a-chip opstelling. Hierbij wordt gebruik gemaakt van het mengen van vier lichtgolven om een sterke pomppuls om te zetten in een signaal- en idlerpuls die gesynchroniseerd zijn in tijd en qua bandbreedte en piekvermogen geschikt zijn voor CARS-spectroscopie en -microscopie. De pomppuls heeft een vaste golflengte van $1064 \mathrm{~nm}$ en de tijdsduur van $10 \mathrm{ps}$ is gekozen om de bandbreedte van de gegenereerde pulsen overeen te laten komen met de bandbreedte van een gemiddelde vibratieovergang. Een laagvermogen, continue, verstembare laser wordt gebruikt om de frequenties van de signal- en idlerpulsen vast te leggen en door verstemming overeen te laten komen met verschillende vibratieovergangen. Uit het model volgt dat het voor niet-lineaire golflengtegeneratie benodigde ingangspompvermogen meer dan één orde van grootte lager is dan in aanpakken 
gebaseerd op optische vezels. Het gevonden bereik voor de golflengteomzetting is $1418 \mathrm{~nm}$ tot $1518 \mathrm{~nm}$ (idler) en $788 \mathrm{~nm}$ tot $857 \mathrm{~nm}$ (signaal). Dit komt overeen met vibratieovergangen van $2350 \mathrm{~cm}^{-1}$ tot $2810 \mathrm{~cm}^{-1}$. Het maximale rendement van $19,1 \%$ wordt verkregen bij een pompvermogen van $300 \mathrm{~W}(\approx$ $2,8 \mathrm{~nJ})$.

Voor het gelijkmaken van de fase van de golven die betrokken zijn bij een niet-lineaire optische proces, zoals het mengen van vier golven (zie hoofdstuk 3) en supercontinuümgeneratie (zie hoofdstukken 5 en 6), is het belangrijk dat de pompgolflengte dicht bij de golflengte ligt waarvoor geen dispersie optreed (ZDW). Het blijkt dat $\mathrm{Si}_{3} \mathrm{~N}_{4} / \mathrm{SiO}_{2}$-golfgeleiders een $\mathrm{Si}_{3} \mathrm{~N}_{4}$-laagdikte van meer dan 0,6 $\mu \mathrm{m}$ moeten hebben om de ZDW-golflengte in de buurt van de golflengte van veel voorkomende lasers te brengen. Echter, het produceren van dergelijke laagdikten vormt een uitdaging voor de gebruikelijke fabricagemethoden om $\mathrm{Si}_{3} \mathrm{~N}_{4}$-golfgeleiders te maken. Het gebruik een lage druk chemisch opdampprocess (LPCVD), dat $\mathrm{Si}_{3} \mathrm{~N}_{4}$ golfgeleiders met de beste optische eigenschappen oplevert, resulteert in een trekspanning welke toeneemt met de laagdikte. Deze toenemende trekspanning resulteert in de vorming van scheuren in de $\mathrm{Si}_{3} \mathrm{~N}_{4}$ laag bij diktes van meer dan 0,4 $\mu \mathrm{m}$ en deze scheuren reduceren de produktieopbrengst aanzienlijk. In hoofdstuk 4 presenteren wij een nieuwe fabricagetechniek voor $\mathrm{Si}_{3} \mathrm{~N}_{4} / \mathrm{SiO} 2$-golfgeleiders met laagdiktes tot $0,9 \mu \mathrm{m}$ waardoor deze geschikt zijn voor niet-lineaire optische toepassingen. De toename in laagdikte is bereikt door eerst gleuven in het thermisch geoxideerd siliciumsubstraat te etsen en vervolgens deze gleuven te vullen met $\mathrm{Si}_{3} \mathrm{~N}_{4}$. Door de congruente groei van LPCVD $\mathrm{Si}_{3} \mathrm{~N}_{4}$ groeien de lagen gelijkmatig vanaf de onderkant en zijkanten van de gleuf totdat de $\mathrm{Si}_{3} \mathrm{~N}_{4}$-lagen van de zijkanten samenkomen en de gleuf uniform gevuld is. Om de uiteindelijke golfgeleiderkern te verkrijgen wordt het overbodige $\mathrm{Si}_{3} \mathrm{~N}_{4}$ buiten de gleuf verwijderd en een mantel van $\mathrm{SiO}_{2}$ aan de bovenzijde gedeponeerd. In $\mathrm{Si}_{3} \mathrm{~N}_{4}$-golfgeleiders die geproduceerd zijn met deze techniek zijn geen scheuren waargenomen, en het process leidt tot een nul uitval van de produktie tengevolge van scheurvorming. De nieuwe golfgeleiders hebben lage propagatieverliezen $(0,4 \mathrm{~dB} / \mathrm{cm}$ bij een golflengte rondom $1550 \mathrm{~nm}$ ) wat een lange interactielengte mogelijk maakt. Verder is het licht geconcentreerd in de kern van de golfgeleider waardoor de berekende verliezen bij buiging van de golfgeleider zeer klein zijn en dus een hoge dichtheid van optische circuits op een chip mogelijk is.

De golfgeleiders, die volgens het in hoofdstuk 3 beschreven proces gefabriceerd zijn, worden gebruikt voor het genereren van een breedbandige supercontinuüm gedreven door een ultrakorte pomppuls met een centrale golflengten van respectievelijk $1560 \mathrm{~nm}$ (hoofdstuk 5) en $1064 \mathrm{~nm}$ (hoofdstuk 6). In hoofdstuk 5 hebben we een mode-locked, Erbium-gedoteerde glasvezellaser met een pulsduur van 65 fs en een pulsenergie van 43 pJ gebruikt om een supercontinuümspectrum met een bandbreedte van meer dan $700 \mathrm{~nm}(-30 \mathrm{~dB})$ te genereren, door te pompen in het regime met anomale groepssnelheidsdispersie. We hebben een uitstekende overeenstemming gevonden tussen het experimenteel gemeten spectrum en berekend spectrum volgens een model gebaseerd op de 
GNLSE. De modelering van het systeem laat zien dat een toename van de pompenergie tot boven de experimentele waarde, maar nog steeds ver onder de beschadigingsgrens van de golfgeleider, leidt tot een supercontinuüm met een bereik van meer dan één octaaf en een hoge mate van coherentie over de gehele gegenereerde bandbreedte voor een pulsenergie vanaf 50 pJ. Een dergelijk supercontinuüm kan bijvoorbeeld gebruikt worden als optische frequentiekam.

Het in hoofdstuk 5 beschreven supercontinuüm loopt van $1225 \mathrm{~nm}$ tot $1960 \mathrm{~nm}$ wanneer de golfgeleider gepompt wordt nabij de langste golflengte van de twee ZDWs. Om het spectrum van het supercontinuüm uit te breiden naar kortere golflengten - wat nodig is voor bijvoorbeeld bepaalde biologische toepassingen - moet het supercontinuüm gepompt worden nabij de kortste golflengte van de twee ZDW's. In hoofdstuk 6 staat beschreven hoe wij een mode-locked Ytterbium-gedoteerde glasvezellaser met centrale golflengte van $1064 \mathrm{~nm}$ en een pulsduur van 115 fs hebben gebruikt om een ultrabreed supercontinuüm te genereren dat het grootste deel van het transmissievenster van $\mathrm{Si}_{3} \mathrm{~N}_{4}$-golfgeleiders beslaat. Bij een ingekoppelde pulsenergie van $590 \mathrm{pJ}$ strekt het gegenereerde spectrum zich uit van het zichtbare (vanaf $470 \mathrm{~nm}$ ) tot het infrarood $(2130 \mathrm{~nm})$ gedeelte van het spectrum $(-30 \mathrm{~dB})$. De spectrale bandbreedte is groter dan $495 \mathrm{THz}$, wat op dit moment het breedste supercontinuümspectrum is dat op een chip gegenereerd wordt. Om de nauwkeurigheid van ons numerieke model voor supercontinuümgeneratie te verbeteren hebben we het model van hoofdstuk 2 uitgebreid door het optisch veld te beschrijven als een superpositie van een aantal transversale modi, aangezien bij een pompgolflengte van $1064 \mathrm{~nm}$ de golfgeleider meer dan alleen de fundamentele transversale mode ondersteunt. Met behulp van dit model laten we zien dat na een voortplanting over slechts $2 \mathrm{~mm}$ het grootste deel van de bandbreedte van het supercontinuüm al gerealiseerd is. Dit laat zien dat niet-lineaire frequentieomzetting in siliciumnitride-golfgeleiders zeer compacte en efficinte instrumenten mogelijk maakt.

De resultaten gepresenteerd in dit proefschrift laten zien dat $\mathrm{Si}_{3} \mathrm{~N}_{4} / \mathrm{SiO}_{2}$ golfgeleiders veel potentie hebben voor on-chip, niet-lineaire frequentieomzetting, dat toegepast kan worden in compacte en veelzijdige geïntegreerde lichtbronnen voor talrijke toepassingen. 



\section{List of publications}

\section{Peer-reviewed journals}

- J.P. Epping, T. Hellwig, M. Hoekman, R. Mateman, A. Leinse, R.G. Heideman, A. van Rees, P.J.M. van der Slot, C.J. Lee, C. Fallnich, and K.-J. Boller, "Broadband supercontinuum generation at telecommunication wavelengths in dispersion engineered stoichiometric silicon nitride waveguides," submitted for publication.

- J.P. Epping, T. Hellwig, M. Hoekman, R. Mateman, A. Leinse, R.G. Heideman, A. van Rees, P.J.M. van der Slot, C.J. Lee, C. Fallnich, and K.-J. Boller, "On-chip visible-to-infrared supercontinuum generation with more than $495 \mathrm{THz}$ spectral bandwidth," Optics Express 23(15), 19596 (2015).

- T. Hellwig, J.P. Epping, M. Schnack, K.-J. Boller, and C. Fallnich, "Ultrafast, low-power, all-optical switching via birefringent phase-matched transverse mode conversion in integrated waveguides," Optics Express 23(15), 19189 (2015).

- J.P. Epping, M. Hoekman, R. Mateman, A. Leinse, R.G. Heideman, A. van Rees, P.J.M. van der Slot, C.J. Lee, and K.-J. Boller, "High confinement, high yield $\mathrm{Si}_{3} \mathrm{~N}_{4}$ waveguides for nonlinear optical applications," Optics Express 23(2), 642 (2015).

- J.P. Epping, M. Kues, P.J.M. van der Slot, C.J. Lee, C. Fallnich, K.-J. Boller, "Integrated CARS source based on seeded four-wave mixing in silicon nitride," Optics Express 21(26), 32123 (2013). 


\section{International conferences}

- J.P. Epping, T. Hellwig, R. Mateman, A. van Rees, M. Hoekman, A. Leinse, R.G. Heideman, P.J.M. van der Slot, C.J. Lee, C. Fallnich, and K.-J. Boller, "Simultaneous Supercontinuum and Third Harmonic Generation in $\mathrm{Si}_{3} \mathrm{~N}_{4}$ waveguides," European Conference on Lasers and ElectroOptics and the International Quantum Electronics Conference, Munich, Germany (2015).

- J.P. Epping, T. Hellwig, R. Mateman, A. van Rees, M. Hoekman, A. Leinse, R.G. Heideman, P.J.M. van der Slot, C.J. Lee, C. Fallnich, and K.-J. Boller, "Supercontinuum generation in thick silicon nitride waveguides," 6th EPS-QEOD Europhoton Conference, Neuchâtel, Switzerland (2014).

- T. Hellwig, J.P. Epping, M. Schnack, K.-J. Boller, and C. Fallnich, "Low-energy, all-optical switching of transverse modes in integrated waveguides," 6th EPS-QEOD Europhoton Conference, Neuchâtel, Switzerland (2014).

- J.P. Epping, M. Kues, P.J.M. van der Slot, C.J. Lee, C. Fallnich, and K.J. Boller, "Integrated Four-Wave Mixing Source for Coherent anti-Stokes Raman Scattering Based on Silicon Nitride," European Conference on Lasers and Electro-Optics and the International Quantum Electronics Conference, Munich, Germany (2013)

- J.P. Epping, M. Kues, P.J.M. van der Slot, C.J. Lee, C. Fallnich, and K.-J. Boller, "Numerical modeling of seeded FWM in silicon nitride waveguides for CARS," XXI International Workshop on Optical Wave \& Waveguide Theory and Numerical Modelling, Enschede, The Netherlands (2013). 


\section{Acknowledgments}

During the last four years there were numerous people who contributed to the work that now forms my PhD thesis, whether they are aware of that or not. This last section is now dedicated to all people that helped me during these years.

First of all, I want to thank my promoter Klaus Boller for giving me the opportunity to do this research in the first place. Our discussions helped me to understand things I did not know and even more to realize that I do not yet understand things I thought I knew. The latter is a lesson I will always keep in mind. Furthermore, I thank my second promoter, Carsten Fallnich, for welcoming me in his group, where I could do part of my research. He also once said that he looks at the acknowledgments first, which is the reason I am mentioning it here.

Both of my supervisors, Peter and Chris, deserve my gratitude as they always helped me when I approached them with questions and they gave constructive feedback to all of my work.

I would like to thank my family for their support and most of all Nina, who in the meantime became my wife. A lot of support was needed due to countless hours of commuting between Münster and Enschede.

Furthermore, a huge credit of the work done during the last four years goes to the people working at LioniX, who planned with me and supplied me with the waveguides so badly needed for my research by pushing the boundaries of the material. So thanks: Marcel, Arne, Richard, Albert, Remco, Willem, and René.

Also my paranynmphs, Tim and Marco, who spared neither trouble nor expense in order to assist me in my defense. It was a pleasure working with the two of you.

The research group, Laser Physics and Nonlinear Optics, deserves many thanks as I enjoyed working with all of them in the last four years. It was really fun to work, joke, discuss, and throw stress balls at you. So thank you: Ab, Ale, Bert, Carin, Caterin A and B, Fred, Gerard, Ingrid, Jean, Jesse, Jonathan, Kai, Kasper, Marc, Marcel, Muharrem, Ruud, Thomas, Wiebke, Yin, and last but not least Youwen, our biggest Fan. Keep up the PhD diners, I really enjoyed the time with you. Tom as well as Peter deserve special thanks for translating the summary to Dutch, which, to my shame, I could not. Also Ruud has to be acknowledged for supplying me with the template for this 
document that I shamelessly used. Furthermore, my bachelor students, also known as the lab monkeys, Boudewijn and Karel, Bob and Reinoud, Paul and Ron deserve mentioning as working with you was fun. I also want to thank all the current and former members of my part-time group, optical technologies, at the University of Münster who worked with me and especially Michael Kues, who helped me to setup the first simulations on nonlinear optical processes in our waveguides. Further thanks go to the members of our neighboring research group, COPS, for the nice discussions during coffee time and for sharing cake. I will always keep Hasan's laughter in mind.

At last I want to thank all the people with whom I enjoyed my spare time: our weekly D\&D group (Carsten, Christina, Hanno, and Peter), my scouting group, and all the people with whom I played field hockey in past years.

Jörn P. Epping

Münster

August 10, 2015 
